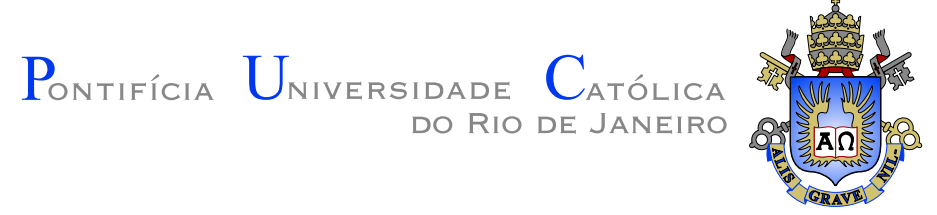

Michael Moraes Cândido

\title{
Problemas em condutividade térmica em cadeias harmônicas e anarmônicas
}

Tese apresentada ao Programa de Pós-graduação em Física do Departamento de Física da PUC-Rio como requisito parcial para obtenção do grau de Doutor em Física.

Orientador: Prof. Welles Antonio Martinez Morgado Co-Orientador: Prof. Sílvio Manuel Duarte Queirós 


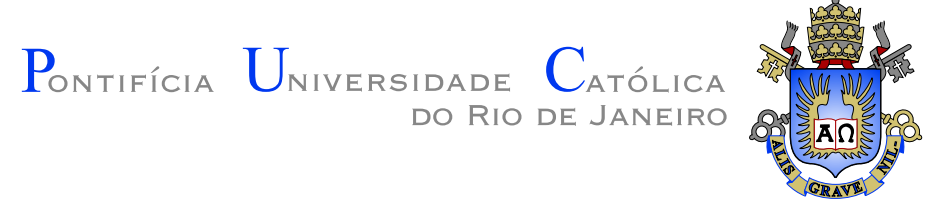

\section{Michael Moraes Cândido}

\section{Problemas em condutividade térmica em cadeias harmônicas e anarmônicas}

Tese apresentada ao Programa de Pós-graduação em Física do Departamento de Física do Centro Técnico Científico da PUCRio como requisito parcial para obtenção do grau de Doutor em Física. Aprovada pela comissão examinadora abaixo assinada.

Prof. Welles Antonio Martinez Morgado

Orientador

Departamento de Física - PUC-Rio

Prof. Sílvio Manuel Duarte Queirós

Co-Orientador

CBPF

Profa. Celia Beatriz Anteneodo de Porto

Departamento de Física - PUC-Rio

Prof. Luca Moriconi

UFRJ

Prof. Daniel Adrian Stariolo

UFF

Prof. Thiago Barbosa dos Santos Guerreiro

Departamento de Física - PUC-Rio

Prof. Márcio da Silveira Carvalho

Coordenador Setorial do Centro Técnico Científico PUC-Rio

Rio de Janeiro, 11 de abril de 2017 
Todos os direitos reservados. É proibida a reprodução total ou parcial do trabalho sem autorização da universidade, do autor e do orientador.

\section{Michael Moraes Cândido}

Atua principalmente no seguinte tema: física de não equilíbrio. Processos Estocásticos

Ficha Catalográfica

Cândido, Michael Moraes

Problemas em condutividade térmica em cadeias harmônicas e anarmônicas / Michael Moraes Cândido; orientador: Welles Antonio Martinez Morgado; co-orientador: Sílvio Manuel Duarte Queirós. — 2017.

v., 109 f: il. color. ; $30 \mathrm{~cm}$

1. Tese (doutorado) - Pontifícia Universidade Católica do Rio de Janeiro, Departamento de Física.

Inclui bibliografia

1. Física - Teses. 2. Condutância térmica. 3. Condutividade térmica. 4. Ruído de Poisson. 5. Método de Fourier-Laplace. 6. Anomalias térmicas. I. Martinez Morgado, Welles Antonio. II. Duarte Queirós, Sílvio Manuel. III. Pontifícia Universidade Católica do Rio de Janeiro. Departamento de Física. IV. Título. 


\section{Agradecimentos}

Gostaria de fazer uma homenagem em primeiro lugar à minha avó, Maria de Lourdes, que me ensinou sobre a importância do estudo na vida de uma pessoa. Nada do que fiz até hoje no âmbito acadêmico seria possível se não fosse o apoio de minha avó.

Ao meu orientador Welles Morgado, por todos os conselhos e ensinamentos passados ao longo dos anos de doutorado. Obrigado também pela confiança depositada no meu trabalho e paciência demonstrada nas inúmeras conversas onde eu aparecia com uma enxurrada de dúvidas em sua sala.

Ao meu co-orientador Sílvio Queirós, pela extrema dedicação em nosso projeto. Reuniões no CBPF seguidas por fatias de cheesecake, discussões via Skype, troca de emails durante madrugadas, enfim, só me resta agradecer por todo o suporte que me foi dado.

Aos meus amigos do IF-UFRJ, Anderson Kendi, Carlos Zarro, Daniel Kroff, Daniel Niemeyer, Elvis Soares, José Apaza, Marcelo Vargas, Maurício Hippert, Pedro Malta e Reinaldo de Melo, que me acompanham na minha trajetória na física desde o princípio. Muito obrigado pela amizade de vocês!

Aos meus amigos da PUC-Rio Carlos Olivares, Eduardo Colombo, Júlio Nicolini e Lucianno Defaveri, que me acompanharam durante esta jornada sempre me dando o apoio necessário.

À minha noiva e futura esposa, Karen, por todas as ocasiões que tivemos que nos privar de momentos de diversão para que eu pudesse trabalhar. Obrigado pela sua compreensão, seu amor e companheirismo incondicionais :).

Gostaria de agradecer as minhas tias Carla e Mariana, e a minha irmã Esther, minhas amigas de toda vida, que sempre estiveram ao meu lado me dando apoio.

Aos meus pais, Paula e Márcio, por tudo que fizeram por mim ao longo da minha vida. Obrigado por sempre acreditarem em mim e pela motivação que vocês me dão em qualquer circunstância.

Ao $\mathrm{CNPq}$ pelos auxílios concedidos, sem os quais este trabalho não poderia ter sido realizado. 


\section{Resumo}

Cândido, Michael Moraes; Martinez Morgado, Welles Antonio; Duarte Queirós, Ślvio Manuel. Problemas em condutividade térmica em cadeias harmônicas e anarmônicas. Rio de Janeiro, 2017. 109p. Tese de Doutorado - Departamento de Física, Pontifícia Universidade Católica do Rio de Janeiro.

No presente trabalho faz-se uma análise sobre quantidades estatísticas de cadeias lineares e não lineares na situação em que o fluxo de calor que atravessa estes sistemas encontra-se no regime estacionário. A discussão inicial é feita sobre um modelo geral de cadeia linear, com acoplamentos arbitrários entre suas partículas e alimentada por reservatórios gaussianos. Uma análise detalhada sobre quantidades como fluxo de calor e distribuição de temperaturas do sistema é feita, onde todas as expressões analíticas correspondentes a estas quantidades são demonstradas e comparadas com resultados numéricos. Estudam-se então as mudanças quantitativas e qualitativas apresentadas pelas grandezas supracitadas quando modificam-se os acoplamentos de ancoragem entre o sistema e os reservatórios. Verifica-se que as mudanças nos perfis de temperaturas estão relacionadas aos extremos dos cumulantes do fluxo de calor, o que motiva uma investigação sobre a possível ocorrência de uma transição de fase no sistema. Buscando encontrar possíveis comportamentos críticos, definem-se as funções de correlação entre as velocidades quadráticas e de velocidades entre pares de partículas. A partir destas definições é possível verificar o comprimento de correlação associado à estas grandezas. Este estudo leva a um dos pontos mais interessantes do trabalho, onde conectam-se as mudanças apresentadas por grandezas do sistema como quantidades estatísticas do fluxo de calor, distribuição de temperaturas do sistema e os seus modos vibracionais frente às mudanças nos acoplamentos de ancoragem com os reservatórios. Ao estudar o fenômeno de condução de calor de uma forma mais realística e rigorosa, é imprescindível acrescentar interações não-lineares na cadeia. Considerando que a solução exata para este tipo de sistema não pode ser obtida, utiliza-se teoria de perturbação e outras ferramentas matemáticas para discutir as principais caracterísiticas do fluxo de calor em uma cadeia anarmônica. A técnica desenvolvida nesta tese permite calcular o fluxo de calor em cadeias de tamanho arbitrário, e é válida para sistemas sob ação de reservatórios de qualquer natureza. Aplica-se o método para cadeias alimentadas por reservatórios gaussianos e poissonianos, de onde verifica-se o impacto das não linearidades sobre estes sistemas e comparam-se os resultados obtidos com o caso linear. Para a análise em que o reservatório poissoniano injeta energia 
no sistema, ilustra-se o efeito de cumulantes de ordem superior do ruído descontínuo sobre o fluxo de calor e como estes novos elementos podem levar a resultados que a primeira vista parecem fisicamente incoerentes.

\section{Palavras-chave}

Condutância térmica ; Condutividade térmica ; Ruído de Poisson ; Método de Fourier-Laplace ; Anomalias térmicas. 


\section{Abstract}

Cândido, Michael Moraes; Martinez Morgado, Welles Antonio (advisor); Duarte Queirós, Sílvio Manuel. Problems in thermal conductivity for harmonic and anharmonic chains. Rio de Janeiro, 2017. 109p. Tese de Doutorado - Departamento de Física, Pontifícia Universidade Católica do Rio de Janeiro.

In the present work I make an analysis about statistical quantities for linear and nonlinear chains in the stationary state. We start the discussion from a general linear model, with arbitrary couplings and connected to Gaussian reservoirs. A detailed analysis for quantities like heat flow and site temperatures is obtained, where all analytical expressions respective to those quantities are derived and a compared with numerical results. Then I study the quantitative and qualitative changes presented by the aforementioned quantities when the pinnings related to the reservoirs are modified. The changes in temperature profiles are related with the extrema of heat flux cumulants, motivating the investigation of whether phase transitions in the chain might occur. In order to investigate possible critical behaviors, I define velocity correlation functions between pair particles and squared velocities correlation functions. From where, one is able to estabilish a correlation length respective to these quantities. This study leads to one of the most remarkable achievements of this work, which is the connection made between the changes presented by some important statistical quantities of heat flux, the system's temperature, vibrational modes and the reservoirs pinnings. By treating the phenomenon of heat conduction in a more realistic and rigorous way, I develop a study to describe the transport properties in an anharmonic chain. Pondering that an exact solution for this sort of system is unfeasible, I use perturbation theory and other mathematical tools to discuss the main features of heat flux in a nonlinear chain. The technique developed throughout this thesis allows one to compute the heat current for a chain of arbitrary size, and is valid for systems under influence of reservoirs of any nature. We apply the method for chains governed by Gaussian and Poissonian reservoirs, verifying the impact of the nonlinearities over those systems, and comparing the obtained results to the linear case. In the case where there is a Poissonian bath injecting energy into the system, I shed some light on the effects of higher order cumulants related to the discontinous noise in the heat flux and I show how these new elements can lead to some results that at first glance seem physically incoherents.

\section{Keywords}


Thermal conductance ; Thermal conductivity ; Poisson noise ; Fourier-Laplace method ; Thermal anomalies.

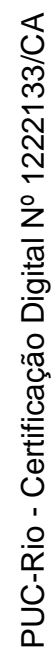




\section{Sumário}

1 Introdução 14

2 Fluxo de calor em uma cadeia harmônica linear $\quad 22$

2.1 Transmissão de calor via condução 22

2.2 A lei de Fourier 23

2.3 O modelo estudado 24

2.4 Calculando a transmissão de energia no sistema 26

2.5 Exemplos numéricos e discussão dos resultados 31

3 O papel das correlações entre as velocidades nos perfis de temperatura $\begin{array}{ll}\text { do sistema } & 37\end{array}$

3.1 Possíveis perfis de temperatura de uma cadeia harmônica linear 37

3.2 Funções de correlação e modos de vibração em uma cadeia 42

$4 \quad$ O papel das anarmonicidades no fluxo de calor em cadeias alimentadas por reservatórios térmicos e atérmicos $\quad 52$

4.1 Reservatórios gaussianos (térmicos) 55

4.2 Reservatórios poissonianos (atérmicos) 57

$\begin{array}{lll}4.3 & \text { Reservatórios gaussianos e poissonianos } & 59\end{array}$

5 Conclusões e perspectivas futuras $\quad 63$

A A transformada de Fourier-Laplace e sua aplicação no cálculo do fluxo de calor $\quad 69$

$\begin{array}{lll}\text { B } & \text { Os operadores } \mathcal{D}(s) \text { e } \mathcal{A}(s) & 71\end{array}$

$\begin{array}{ll}\text { C Fluxo de calor no limite termodinâmico } & 73\end{array}$

$\begin{array}{lll}\text { D Correlações de velocidades no limite termodinâmico } & \mathbf{7 8}\end{array}$

E Desenvolvimento perturbativo do caso não linear 82

E.1 Desenvolvimento do termo II 83

E.2 Desenvolvimento do termo $I \quad 86$

E.3 Temperatura das partículas da cadeia 93

F $\quad$ Resultados suplementares dos cumulantes da cadeia harmônica linear $\quad \mathbf{9 7}$ 
Referências bibliográficas

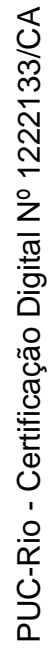




\section{Lista de figuras}

1.1 Trajetórias direta e reversa de um sistema fora de equilíbrio no espaço de fases.

2.1 Visão pictórica da cadeia harmônica com ancoragem. Embora as molas relacionadas ao parâmetro de ancoragem estejam dispostos transversalmente na figura, eles estão associados a vibrações longitudinais.

2.2 Disposição dos pólos da dinâmica do problema do exemplo em que $N=2$. Os termos $i\left(\epsilon+r_{1}\right), i\left(\epsilon+r_{2}\right), i\left(\epsilon+r_{3}\right)$ e $i\left(\epsilon+r_{4}\right)$ representam os pólos da função $\widetilde{\mathcal{A}}_{1 N}(\mathrm{i} q+\varepsilon)$, enquanto os termos $i\left(\epsilon-r_{1}\right), i\left(\epsilon-r_{2}\right), i\left(\epsilon-r_{3}\right)$ e $i\left(\epsilon-r_{4}\right)$ representam os pólos não integráveis de $\widetilde{\mathcal{A}}_{1 N}(-\mathrm{i} q-\varepsilon)$.

2.3 Gráfico descrevendo a condutância versus $\frac{1}{N}$.

2.4 A figura ilustra qualitativamente o potencial elástico de um cristal em duas situações: i) região onde a expansão harmônica é razoável e a energia do sistema é dada por $E_{1}$; ii) região onde os efeitos das não linearidades devem ser levados em consideração com energia $E_{2} .35$

3.1 As figuras (a), (b), e (c) mostram os possíveis perfis de temperatura em uma cadeia linear. Aqui tomou-se $k_{1}=k^{\prime}=\gamma=m=1 \mathrm{e}$ uma cadeia com $\mathrm{N}=20$. As temperaturas dos reservatórios térmicos correspondem as linhas verde $\left(T_{1}=1\right)$ e vermelha $\left(T_{N}=2\right)$.

3.2 Temperatura da partícula $2, \mathcal{T}_{2}$, vs ancoragem de volume, $k$. com $m=1, \gamma=1$. A linha azul cheia corresponde aos parâmetros $k^{\prime}=k_{1}=1$ e a linha vermelha tracejada-pontilhada está associada a $k^{\prime}=k_{1}=2$. Os valores críticos de $k$ são, $k_{\text {crit }}=1 / 2$ e $k_{\text {crit }}=1$, respectivamente, de acordo com Eq. (3-4). Para valores de $k$ tal que $k \rightarrow \infty$, a cadeia se transforma em um fio rígido e a temperatura do volume naturalmente tende a $3 / 2$.

3.3 Regiões do espaço onde a cadeia apresenta perfil de temperaturas do modelo RLL (cúspides) e do modelo estudado. As linhas vermeIha e azul representam, respectivamente, a $1^{\mathrm{a}}$ e $2^{\mathrm{a}}$ transição entre os regimes de temperatura. Ilustra-se também na figura um ponto correspondente ao regime $\mathrm{RLL}$, onde $k^{\prime}=k_{1}=1$ e $k=0$. 
3.4 Cumulantes normalizados versus $k^{\prime}$. Nota-se que todos os cumulantes atingem o extremo justamente no ponto em que o sistema atinge a primeira mudança de regime.

3.5 Comparação dos resultados numéricos e analíticos para os primeiros quatro momentos dos fluxo de calor. Os gráficos ilustram os momentos normalizados pelos seu respectivos valores máximos versus a ancoragem de borda, onde usou-se $m=1, \gamma=1$ e $k=1$, $k_{1}=1$. Os painéis superiores esquerdo e direito ilustram $\langle J\rangle \mathrm{e}\left\langle J^{2}\right\rangle$, respectivamente, enquanto os painéis inferiores e direito mostram $\left\langle J^{3}\right\rangle$ e $\left\langle J^{4}\right\rangle$. As linhas cheias representam a curva analítica e as circunferências os pontos obtidos através da simulação numérica. Para este conjunto de parâmetros os momentos atingem o seu máximo para $k_{\text {crit }_{1}}^{\prime} \approx 1.6180$.

3.6 Comprimento de correlação como função de $\Delta k^{\prime}$. Os parâmetros utilizados são: $m=k_{1}=\gamma=1, k=\frac{1}{2}, T_{1}=1$ e $T_{N}=2$. Os pontos são obtidos analiticamente, e a reta traçada corresponde ao ajuste linear feito, cuja a inclinação é igual a $-0.61576 \pm 10^{-5}$ e o com o comprimento de correlação na origem igual a $\xi^{0}=$ $0.779796 \pm 10^{-6}$.

3.7 Correlação entre as velocidades de pares da cadeia, conforme indicado no lado direito de cada painel. Nos painéis superiores $k^{\prime}=1$ todas as correlações são nulas, e os elementos do volume encontram-se no platô de temperaturas. Quando $k^{\prime}=5 / 2$, o platô de temperaturas ressurge, bem como as correlações entre pares do volume se anulam, sendo as únicas exceções dadas por $C_{v_{1} v_{2}} \mathrm{e}$ $C_{v_{N} v_{N-1}}$. Observe que as correlações são simétricas com relação ao meio da cadeia. Aqui utilizou-se uma cadeia com $\mathrm{N}=11$ elementos.

3.8 Os painéis superiores (3.8(a) e 3.8(b)) mostram que as correlações atingem valor zero nas vizinhanças de $k_{\text {crit }_{1}}^{\prime}=1$. Nos painéis inferiores (3.8(c) e 3.8(d)) é possível notar que os resultados de correlações simultaneamente nulas em $k_{\text {crit }_{2}}^{\prime}=\frac{5}{2}$ não ocorrem na cadeia não-linear. Considerou-se uma cadeia com $\mathrm{N}=11$ elementos nesta análise.

4.1 Cadeia contendo interações harmônicas e anarmônicas entre seus elementos. 
4.2 A corrente de calor para um sistema com $N=10$ partículas. A linha azul contnua representa o resultado analítico, enquanto os círculos vermelhos fornecem os dados da simulação Toma-se os parâmetros com os seguintes valores: $\gamma=m=k^{\prime}=k_{1}=1$ e $k=\frac{1}{2}$.

4.3 O fluxo de calor em uma cadeia com $N=10$ partículas. Faz-se a seguinte escolha para os parâmetros: $T_{N}=2, T_{1}=1.99, \lambda=$ $4, \gamma=m=k^{\prime}=k_{1}=1$ and $k=\frac{1}{2}$.

F.1 Comportamento da variância, obliquidade e curtose do fluxo de calor $J$ em uma cadeia harmônica linear em função do número $N$ de elementos do sistema

F.2 As subfiguras (a), (b) e (c) mostram o comportamento do segundo, terceiro e quarto cumulantes do fluxo de calor ao longo de uma cadeia. As linhas azuis cheias mostram o resultado analítico, enquanto que os quadrados e triângulos representam o resultado da simulação. Utilizou-se $k_{1}=k^{\prime}=\gamma=m=1$. 


\section{1 \\ Introdução}

Os estudos da física de equilíbrio, representam uma área importante para a ciência, e os resultados obtidos através destes estudos trouxeram avanços para muitos problemas físicos, como por exemplo a determinação do calor específico dos materiais [1], a explicação para a radiação de corpo negro [2], as grandezas macroscópicas que definem a função de estado de um gás [3], a descrição da magnetização dos metais [4], estudo sobre transições de fase [5], entre outros exemplos. Todas estas descobertas foram feitas essencialmente empregando os princípios da física de equilíbrio e da mecânica estatística.

Apesar do êxito obtido no desenvolvimento das teorias citadas, existem outros fenômenos importantes fora do escopo da física de equilíbrio, como por exemplo a dinâmica no interior de planetas/estrelas [6, 7], a transmissão de calor [1,3], o transporte eletrônico [8], o processo de bariogênese [9] e sistemas biológicos [10]. Tais fenômenos são tratados utilizando a abordagem da física de não-equilíbrio, uma área ainda não tão bem estabelecida como sua contra-parte de equilíbrio, mas cujo desenvolvimento vem se acelerando bastante devido ao progresso computacional e ao avanço das técnicas experimentais.

Um dos interesses da física de não equilíbrio é descrever de forma precisa os fenômenos termodinâmicos com aplicações práticas tais como, estudo sobre propriedades de transporte do grafeno, medição da condutividade térmica de nanoestruturas cristalinas, medidas quânticas de condução térmica e transmissão de calor em nanotubos de carbono [11-16]. Diferentemente de um sistema termodinâmico em equilíbrio, não é possível utilizar a formulação de Boltzmann-Gibbs, que calcula o volume acessível do espaço de fases do sistema (exponencial da entropia), e sua energia, $\epsilon$, dando assim um peso estatístico a cada um dos possíveis micro-estados do sistema que é proporcional ao fator $e^{\frac{-\epsilon}{k_{B} T}}$. Caso esta descrição fosse aplicável em um sistema fora do equilíbrio, seria possível definir funções de estado como energia livre e entropia, prever diferentes fases termodinâmicas da matéria fora do equilíbrio, ou entender como o sistema responde a perturbações.

Como não é possível aplicar este formalismo, surge a necessidade de utilizar técnicas capazes de explicar os fenômenos da termodinâmica fora do equilíbrio, como por exemplo princípios relacionados à mínima dissipação de energia desenvolvidos por Lars Onsager em 1931 [17, 18] e teoremas de 
flutuação [19, 20].

No trabalho realizado por Onsager mostrou-se que em sistemas termodinâmicos fora do equilíbrio é possível estabelecer a igualdade entre determinadas razões de fluxos e forças pertinentes ao sistema. Por exemplo, considere que o sistema em questão é um fluido descrito em termos de sua temperatura, densidade e pressão. É sabido que a variação de qualquer uma dessas grandezas produz uma corrente no sistema, seja esta de massa ou de calor. As relações de Onsager em um fluido mostram que :

$$
\frac{J}{\Delta p}=\frac{\rho}{\Delta T}
$$

onde $J$ representa o fluxo de calor ao longo do fluido, $\Delta p$ a diferença de pressão entre duas regiões do fluido, $\Delta T$ a respectiva diferença de temperauras entre as regiões e $\rho$ a densidade do fluido. Embora as relações de Onsager sejam mais intuitivas de entender em um fluido, seu trabalho original foi feito para descrever de uma forma mais completa fenômenos de transporte em eletrodos e termoelétricos, tais como os efeitos Peltier e Seeback [17].

O princípio básico no qual se baseiam os teoremas de flutuação é a análise de dois processos físicos diferentes, definidos como processos diretos e reversos. Estes podem ser entendidos de forma simples ao analisar a figura 1.1, onde mostra-se que o processo direto $\left(\gamma_{D}\right)$ está associado à evolução das variáveis dinâmicas no espaço de fases entre dois pontos $x_{D}(0)$ e $x_{D}(\tau)$ para $\tau>0$, enquanto a trajetória reversa $\left(\gamma_{R}\right)$ representa a evolução do processo das variáveis conjugadas $x_{R}(\tau)$ e $x_{R}(0)$ no sentido reverso de tempo, $\tau<0$. Usando o conceito de trajetórias diretas e reversas é possível construir relações que conectam a probabilidade de observação destes eventos nos sentidos direto e reverso, o que permite por exemplo relacionar o trabalho realizado sobre o sistema fora de equilíbrio com as variações de funções termodinâmicas do mesmo, tal como a energia livre [20], e observar a violação da $2^{\text {a }}$ lei da termodinâmica por curtos intervalos de tempo [19]. Além de representar um significativo avanço teórico, os teoremas de flutuação também possuem aplicações como por exemplo a medição do trabalho realizado sobre moléculas de RNA [21]. 


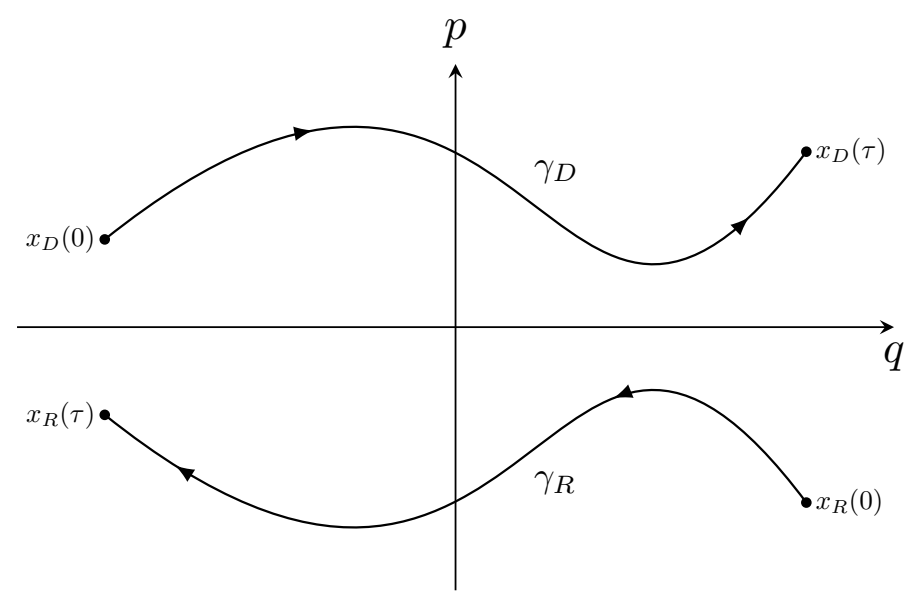

Figura 1.1: Trajetórias direta e reversa de um sistema fora de equilíbrio no espaço de fases.

Dentre as ferramentas para resolver estes problemas relacionados a sistemas fora do equilíbrio pode se destacar o uso dos processos estocásticos, uma teoria que nasceu do estudo de problemas físicos concretos, como por exemplo o movimento browniano, e que hoje possui aplicações em diversas áreas que vão desde motores moleculares até análise de tráfego na internet [22]-[23]. Uma das possíveis aplicações de processos estocásticos por exemplo é a transmissão de calor via condução fonônica, assunto que é descrito neste trabalho no contexto de cadeias unidimensionais.

Embora o transporte de calor via condução térmica seja há muito tempo conhecido, existem muitas questões associadas a este fenômeno ainda não solucionadas. A mais proeminente, provavelmente, está associada ao fato de, até o presente momento, não existir nenhum modelo teórico construído a partir de primeiros princípios, aplicado aos sólidos, que leve corretamente à observação da lei de Fourier, que correlaciona o fluxo de calor ao longo de um sistema com o gradiente de temperatura, em sistemas unidimensionais ou bidimensionais.

Muitos modelos, utilizando uma abordagem clássica, foram criados com intuito de fornecer uma descrição microscópica de tal lei e [2429], embora nenhum deles tenha atingido o principal objetivo, existem características associadas às grandezas macroscópicas destes modelos, tais como condutividade térmica e temperatura, que apresentam comportamentos um tanto contra-intuitivos. Como exemplo de trabalhos pioneiros nesta área pode se citar os modelos desenvolvidos por Nakazawa e Rieder-LebowitzLieb (RLL) [24, 27], onde pode se destacar os resultados sobre o fluxo de calor através de cadeias homogêneas e harmônicas sob influência de banhos térmicos em suas extremidades, sendo estes associados à energia que atravessa 
os sistemas.

Embora os modelos possuam uma sutil diferença entre suas hamiltonianas, ambos levam ao mesmo resultado: um fluxo de calor que independe do tamanho da cadeia. Este resultado é um tanto estranho, pois pode se imaginar que se uma cadeia possui um tamanho "infinito" a corrente de calor que o atravessa deve ser nula, visto que a resistência térmica do sistema também seria infinita. O modelo RLL além de apresentar um fluxo de calor que independe do tamanho do sistema, ainda possui um perfil de temperaturas um tanto contra-intuitivo, onde as partículas da cadeia mais próximas da fonte quente de calor apresentam temperaturas menores que as partículas que se encontram mais próximas à fonte fria de calor! Estes resultados motivam um estudo sobre o porquê destas anomalias, bem como a inserção de possíveis correções nos modelos de forma a eliminá-las, permitindo assim a descrição correta do fluxo de calor em um sólido.

As anomalias citadas são características em modelos de cadeias que possuem interações harmônicas entre primeiros vizinhos, sistemas estudados em cursos de física de estado sólido [30, 31]. A aproximação harmônica envolve basicamente a expansão do potencial elástico de um sólido em torno do seu mínimo, de forma a manter termos até segunda ordem do potencial, i.e., elementos vizinhos da rede possuem interações lineares entre si. Ao analisar um cristal harmônico através de suas coordenadas normais nota-se que suas vibrações podem ser vistas como um conjunto de fônons não interagentes [31]. Uma consequência do não espalhamento de fônons neste tipo de sistema é observada no fluxo de calor $J$ que atravessa o sistema, visto que este independe do número $N$ de partículas que compõem a cadeia [27]. O fato de $J$ ser constante está ligado ao fato do transporte balístico de calor que um sistema linear apresenta, implicando desta maneira em uma condutividade térmica $\kappa$ infinita, justificando assim o resultado encontrado nos modelos propostos por Nakazawa e RLL (ver capítulo 2 para uma discussão mais detalhada).

Tão importante quanto o tipo de interação que os elementos da cadeia possuem entre si é a forma que estes vão interagir com o meio externo, i.e., definir como a energia é injetada no sistema bem como modelar a dinâmica que este possui com os elementos que o delimitam espacialmente. O uso de reservatórios térmicos é muito comum para descrever como o sistema troca calor com o meio externo, podendo estes por exemplo possuírem natureza estocástica [24, 27] ou determinística [32, 33]. Ao longo deste texto opta-se por trabalhar com reservatórios estocásticos que obedecem a equação de Langevin, visto que fisicamente esta é uma boa ilustração dos reservatórios como uma fonte simultânea de ruído e dissipação [34]. Já a modelagem das demais 
interações da cadeia com o meio externo, substrato e bordas, pode ter diferentes naturezas como por exemplo estocástica [35, 36], não-linear [28, 37] e linear [24, 26], sendo esta última a adotada neste trabalho. Os parâmetros associados à interação entre a cadeia e o meio externo externo são denominados constantes de ancoragem (pinnings).

A maior parte dos trabalhos relacionados ao transporte de calor citados estudam propriedades de cadeias unidimensionais (lineares), que embora sejam uma idealização de uma situação física real, mostram-se muito úteis na modelagem/descrição de experimentos de objetos quasi $1 D$, tais como grandes cadeias moleculares, nanotubos e nanofios [13-16, 38-42]. Vale frisar que as anomalias apresentadas pela condutividade térmica não são decorrentes apenas do tipo de interações que o sistema possui mas também de sua dimensão. Experimentos com nanotubos de carbono de parede única indicam a existência de um regime anômalo de transmissão de calor e em nanotubos de carbono com múltiplas paredes, onde verifica-se que a condutividade segue uma lei de potência crescente em termos do comprimento da amostra [43, 44].

Ao estudar propriedades de uma cadeia harmônica linear nota-se que além de propriedades macroscópicas como o fluxo de calor e temperatura das partículas, outras grandezas úteis na descrição global do sistema são suas quantidades estatísticas associadas ao fluxo de calor [45, 46]. Estas informações estatísticas são, basicamente, construídas a partir de cumulantes associados aos banhos térmicos, que são responsáveis pela introdução da estocasticidade no sistema. Grandezas como variância, obliquidade (skewness) e curtose do fluxo de calor são pouco exploradas nos trabalhos que estudam transmissão de calor via condução, bem como as correlações entre os graus de liberdade dos elementos do sistema. Ao longo desta tese explora- se o comportamento destas grandezas frente a mudanças dos parâmetros da dinâmica do sistema, bem como uma descrição do que ocorre com cumulantes de ordem superior da energia injetada na cadeia quando sujeitos a estas mesmas mudanças.

Como será mostrado ao longo do texto, as cadeias puramente lineares representam apenas uma base para um possível modelo de condução de calor. Visando modelar o fenômeno de condução de calor a nível microscópico de forma mais rigorosa e fisicamente consistente, é imprescindível que o sistema estudado possua algum tipo de fonte de espalhamento de fônons, como por exemplo interações anarmônicas, que possibilitam tornar o problema em questão o mais próximo possível da situação real. Em um trabalho desenvolvido por $\mathrm{Hu}$ et al [47] mostrou-se que para cadeias unidimensionais cujos potenciais de ancoragem são quárticos é possível através de simulações numéricas verificar que o sistema apresenta uma condutividade térmica finita, sendo este resultado 
também verificado em modelos que utilizam métodos semi-analíticos para solucionar o problema[48, 49]. É importante frisar que a modelagem da ancoragem nos trabalhos citados, e neste a ser apresentado, supõe que o substrato que interage com a cadeia possui uma massa infinitamente grande, de forma que este não representa uma fonte de perda de energia para o sistema.

Automaticamente, ao se inserir interações não lineares no sistema, as soluções para as equações de movimento deixam de ser exatas, sendo então obtidas apenas através de simulações numéricas ou via métodos perturbativos através dos quais é possível obter uma descrição aproximada para os graus de liberdade do sistema. A partir das soluções obtidas através de teoria de perturbação é possível desenvolver expressões analíticas aproximadas para todas as grandezas macroscópicas associadas às propriedades de transporte e quantidades termoestatísticas do sistema.

No capítulo 2 são explorados os principais resultados associados a propriedades de transporte em uma cadeia harmônica linear. Na primeira parte deste capítulo apresenta-se o modelo usado como base nesta tese e introduz-se o ferramental matemático que permite calcular grande parte das quantidades de interesse do trabalho. A partir de então é possível discutir qual o comportamento do fluxo de energia que atravessa o sistema, mostrando os detalhes que determinam o regime balístico em uma cadeia harmônica.

Já no capítulo 3, faz-se o estudo sobre as temperaturas dos sítios e investiga-se o comportamento de outras grandezas estatísticas que não são usualmente analisadas, tais como funções de correlação de dois pontos e cumulantes do fluxo de calor de ordem superior a dois. Ao longo do capítulo busca-se entender como estas quantidades se comportam quando alteram-se parâmetros ligados à dinâmica do sistema, como por exemplo, a ancoragem de borda.

Um dos primeiros pontos a serem levantados no terceiro capítulo diz respeito ao perfil de temperatura da cadeia, que pode apresentar diferentes configurações da mostrada no já conhecido modelo RLL [27], sendo estas configurações dependentes da escolha da ancoragem de borda/volume. Mostra-se também que a escolha das constantes de ancoragem tem um papel importante nos cumulantes do fluxo de calor $J$, sendo possível descrever qualitativamente o comportamento de todos os cumulantes de $J$ no problema para diversos valores destes acoplamentos.

A parte final do capitulo 3 relaciona a mudança de perfis de temperatura do sistema com os modos vibracionais da cadeia, utilizando para isto o conceito de funções de correlação. Através da análise de tais funções, mostra-se que as manipulações nas constantes de ancoragem do sistema não são apenas ajustes 
mecânicos, mas sim escolhas que acabam por alterar a distribuição de fônons na cadeia.

O capítulo 4 traz um pouco mais de profundidade ao assunto ao inserir não linearidades nas interações do sistema, tornando-o desta maneira mais próximo de um modelo real para a descrição de vibrações em sólidos. A inserção de interações de natureza não-linear impede que as novas equações de movimento sejam resolvidas de forma analiticamente exata, forçando desta maneira a busca de soluções alternativas para estas. Para lidar com as não linearidades emprega-se o método da teoria de perturbação, o que permite encontrar a solução aproximada para as equações de movimento do sistema e assim obter as mesmas quantidades calculadas no caso linear.

A expansão perturbativa aplicada neste trabalho é feita até primeira ordem, e sua implementação é usada para estudar o fluxo de calor em sistemas alimentados por reservatórios gaussianos, poissonianos e um caso misto, onde ambos encontram-se presentes. A ideia em cada destes casos é verificar como o fluxo de calor se comporta para diversos tamanhos de cadeias e também checar os limites da expansão perturbativa empregada. Explora-se um pouco mais o caso em que os dois tipos de reservatórios estão presentes, investigando qual o papel das não-linearidades nesta situação e quais os efeitos do acoplamento destas com cumulantes de ordem maior que dois, sendo estes últimos ligados ao reservatório atérmico.

A presença dos cumulantes de ordem superior do ruído ligado ao reservatórios em cadeias não-lineares acaba por produzir resultados que, à primeira vista, podem parecer inconsistentes com as leis fundamentais da termodinâmica. No caso misto a ser mostrado, verifica-se que a partir de uma escolha adequada de parâmetros é possível inverter o sentido do fluxo de calor ao longo de uma cadeia anarmônica. Como será mostrado, tal resultado deve-se ao fato de os cumulantes de ordem superior representarem fontes extras de energia para o reservatório, desempenhando assim um papel importante no transporte de calor ao longo da cadeia. A existência de mais de dois cumulantes na distribuição de probabilidades dos ruídos dos reservatórios implica que estes não podem ter suas temperaturas definidas da maneira tradicional, o que faz com que estes banhos (reservatórios) sejam denominados como banhos de não equilíbrio ou atérmicos.

Finalizando, o capítulo 5 mostra as principais conclusões sobre as propriedades de transporte de calor nas cadeias lineares e não lineares estudadas. Uma breve revisão do que foi apresentado ao longo do texto é feita, de forma a dar uma visão geral sobre o enfoque do trabalho. Alguns tópicos não abordados são apresentados de forma breve e colocados em perspectivas 
futuras, como por exemplo, a reprodutibilidade dos resultados obtidos em cadeias de dimensão maior que 1 .

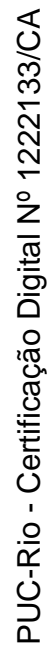




\section{2}

\section{Fluxo de calor em uma cadeia harmônica linear}

\section{1}

\section{Transmissão de calor via condução}

Um dos grandes desafios para a termodinâmica de não-equilíbrio consiste no desenvolvimento de modelos teóricos que conectem o fenômeno de transferência de calor em nível microscópico com o que é observado em níveis macroscópicos. Dentre as formas existentes de transferência de calor, a transmissão via condução representa um mecanismo bem simples de ser entendido de forma qualitativa, porém a teoria por trás deste fenômeno não é trivial. O primeiro passo para compreender o mecanismo de transmissão de calor por condução é identificar como este processo ocorre em materiais de naturezas diferentes.

A transmissão de calor nos fluidos via condução ocorre devido a colisão direta entre as moléculas, e consequentemente, a sua condutividade térmica é pequena quando comparada à dos sólidos visto que o fluido é um meio mais diluto. Por outro lado o transporte de calor nos fluidos possui uma descrição mais complexa, visto que a diferença de temperaturas entre diferentes partes do material também acarreta em uma corrente de convecção no interior do mesmo.

Nos metais a transmissão de calor apresenta uma grande eficiência pois além da energia que é propagada através das vibrações da rede, existe ainda a contribuição dos elétrons livres que transmitem energia ao se deslocarem [8]. Além de apresentarem condutividade térmica elevada, os materiais metálicos também apresentam uma alta condutividade elétrica, sendo estas quantidades correlacionadas pela lei de Wiedemann-Franz, de onde verifica-se que para uma temperatura fixa a razão entre estas grandezas apresenta o mesmo valor para diversos tipos de metais [31].

Já nos materiais não-metálicos a transferência de calor por condução ocorre sem que haja deslocamento líquido do meio no qual a energia se propaga. A transmissão de calor nesta classe de materiais pode ser vista como a propagação das vibrações da rede, onde átomos mais energéticos de uma parte do sólido transferem energia para seus vizinhos menos energéticos através das 
perturbações produzidas na rede. A transmissão destas vibrações é descrita frequentemente em termos dos fônons, que nada mais são do que os quanta associados ao modos normais de vibração do sistema. A ênfase deste trabalho e da maior parte das referências aqui citadas está relacionada a modelos de transporte de calor em materiais que se enquadram nesta última classe descrita, ou seja, onde a transferência de energia é dada apenas pelas vibrações que se propagam ao longo da rede.

\section{2}

\section{A lei de Fourier}

Quando Fourier enunciou a lei da condução térmica há mais de dois séculos atrás (1808), não se pensava que a descrição desta lei em nível microscópico seria uma tarefa tão árdua. A lei de Fourier, que é empiricamente verificada em muitos materiais, enuncia que a corrente de calor através de um sistema em contato com duas fontes de calor de temperaturas diferentes em suas extremidades é proporcional ao negativo do gradiente de temperatura ao longo do sistema. A lei de Fourier pode ser escrita como [34]

$$
J(x, t)=-\kappa \nabla T(x, t),
$$

onde $J(x, t)$ representa o fluxo de calor, $T(x, t)$ a temperatura local e $\kappa$ a condutividade térmica do sistema, uma grandeza que depende do material. Embora esta lei possua muitas aplicações no campo da engenharia, não existe nenhum modelo para sólidos que a comprove em nível microscópico, independente se o modelo em questão utiliza uma abordagem clássica ou quântica [24-28, 35, 50, 51].

Alguns modelos conseguem reproduzir a lei de Fourier em uma dimensão sob a restrição de que o volume do sistema deve estar ligado a reservatórios auto-consistentes [52-55]. Este conceito foi introduzido por Visscher et al em 1970 [56], onde propõe-se que além das fontes de calor nas extremidades da cadeia, existam fontes de calor ligadas ao volume do sistema cuja troca de energia com o mesmo vai a zero assim que o regime estacionário é atingido, ou seja, não existe fluxo de calor médio entre o volume do sistema e os novos reservatórios quando para intervalos de tempo muito grandes. Uma justificativa para introduzir os reservatórios auto-consistentes é de que estes podem ilustrar a interação do volume do sistema com o substrato no qual o sistema se encontra. Embora o resultado obtido neste tipo de modelo seja o desejado fisicamente, a introdução destes reservatórios ao longo do volume do sistema está longe de representar uma formulação mais geral para o problema, de forma que não se dará ênfase a este tipo de modelo ao longo deste trabalho. 
Neste capítulo um estudo detalhado sobre cadeias harmônicas lineares é feito, onde explora-se suas propriedades macroscópicas e estatísticas. Aborda-se também ao longo do restante do texto o papel da influência da escolha dos acoplamentos associados à dinâmica do problema nos comportamentos das grandezas supracitadas, bem como a sua influência na distribuição dos modos de vibração da cadeia.

Um ponto importante a ser frisado é que este trabalho como um todo não possui a meta de construir um modelo do qual seja possível extrair a lei de Fourier. O objetivo principal deste trabalho é estudar propriedades associadas ao transporte de calor em cadeias harmônicas e anarmônicas.

\section{3}

\section{O modelo estudado}

Para descrever a transmissão de calor em termos de modos vibracionais em um sólido no limite de baixas temperaturas, o modelo de interação harmônica representa, pelo menos em primeira análise, um bom toy model, i.e., um modelo extremamente simples utilizado para descrever um fenômeno de maneira qualitativa. É sabido que este tipo de modelo apresenta alguns tipos de propriedades que não condizem com um sistema físico real, tal como transporte de calor balístico, este por sua vez implica em uma condutividade térmica infinita $[27,30,31,34]$. Apesar de apresentar propriedades que não condizem com a de um sólido usual, o modelo harmônico é útil pois ele constitui a base para sistemas físicos um tanto mais complicados como por exemplo o estudo de transmissão de calor em sistemas quânticos [35, 50, 51].

Sistemas harmônicos são bem detalhados na literatura e neste primeiro capítulo faz-se uma discussão sobre uma de suas caracterísiticas mais marcantes: a divergência da condutividade térmica. Estes modelos também são muito importantes pois todo o seu tratamento matemático pode ser feito de forma analítica e exata. Um dos modelos precursores no estudo da transmissão de calor foi proporsto por Rieder, Lebowitz e Lieb (RLL) no ano de 1967 [27], onde mostrou-se que uma cadeia harmônica sujeita a banhos de Langevin com temperaturas diferentes e com acoplamento de ancoragem apenas em suas extremidades apresenta uma condutividade térmica que diverge quando o limite termodinâmico é tomado, além de exibir um perfil de temperaturas bastante incomum. No ano de 1970 Nakazawa fez uma variante do modelo proposto por RLL [24], considerando uma cadeia com um acoplamento de ancoragem idêntico em todos os elementos do sistema, porém os resultado obtidos foram bem parecidos com os do modelo predecessor.

O modelo trabalhado neste capítulo é baseado em uma cadeia cujas 
extremidades encontram-se em contato com dois banhos térmicos de Langevin, possuindo temperaturas $T_{1}$ e $T_{N}$, com $T_{N}>T_{1}$. Além da interação harmônica entre as partículas, cuja constante de acoplamento é denotada por $k_{1}$, o sistema possui o acoplamento de ancoragem em todos os sítios, dados por $k$ e $k^{\prime}$, que representam as ancoragens de volume e borda, respectivamente. Uma possível justificativa para a ancoragem ao longo da cadeia é a interação do sistema com o substrato, onde de maneira mais geral admite-se que o acoplamento efetivo das bordas seja diferente do acoplamento de volume. As equações de movimento que regem a dinâmica do sistema são dadas por:

$$
\left\{\begin{array}{l}
m \frac{d^{2} x_{1}}{d t^{2}}=-\gamma \frac{d x_{1}}{d t}-k^{\prime} x_{1}-k_{1}\left(x_{1}-x_{2}\right)+\eta_{1} \\
m \frac{d^{2} x_{i}}{d t^{2}}=-k x_{i}-k_{1}\left(2 x_{i}-x_{i+1}-x_{i-1}\right) \\
m \frac{d^{2} x_{N}}{d t^{2}}=-\gamma \frac{d x_{N}}{d t}-k^{\prime} x_{N}-k_{1}\left(x_{N}-x_{N-1}\right)+\eta_{N}
\end{array}\right.
$$

com $(2 \leqslant i \leqslant N-1)$, onde $\eta$ é o ruído gaussiano associado aos reservatórios térmicos com correlação temporal dada por:

$$
\left\langle\eta_{i}(t) \eta_{j}\left(t^{\prime}\right)\right\rangle=2 \gamma T_{i} \delta_{i j} \delta\left(t-t^{\prime}\right) \quad \text { com } \quad(i, j) \in\{1, N\}
$$

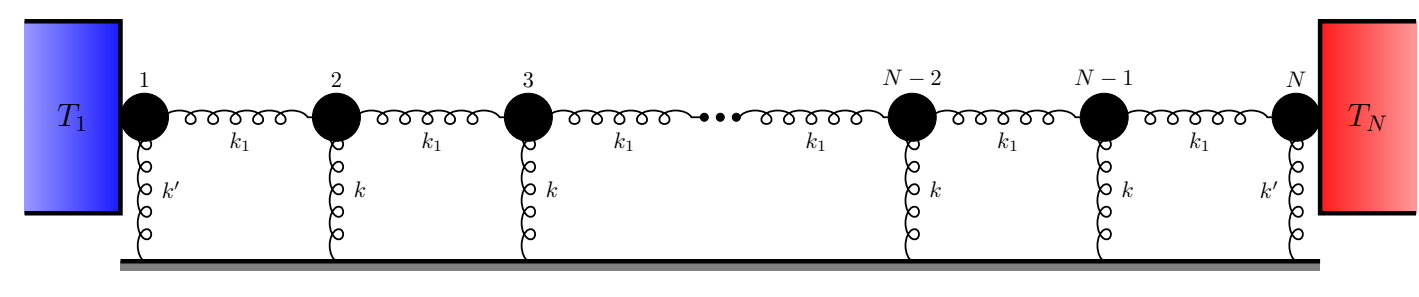

Figura 2.1: Visão pictórica da cadeia harmônica com ancoragem. Embora as molas relacionadas ao parâmetro de ancoragem estejam dispostos transversalmente na figura, eles estão associados a vibrações longitudinais.

Uma vez que as equações de movimento estão determinadas e a natureza dos banhos térmicos é bem definida, o próximo passo é estabelecer uma expressão que quantifique o balanço energético entre o sistema e os reservatórios. 


\section{4}

\section{Calculando a transmissão de energia no sistema}

\subsection{1}

\section{Método I}

Como a variação de energia que ocorre no sistema está associada apenas a sua interação com o banho térmico, a expressão do calor injetado pode ser construída ao se analisar um exemplo mais simples do que a cadeia harmônica, o caso onde existe apenas uma partícula sendo alimentada pelo reservatório. Para esta situação pode se escrever a equação de movimento da partícula como:

$$
m a=-\gamma v-k^{\prime} x+\eta
$$

Pode-se então multiplicar ambos os lados da Eq. (2-4) por $v$ de forma que tem-se

$$
\begin{gathered}
m v a=-\gamma v^{2}-k^{\prime} x v+\eta v= \\
=\frac{d}{d t}\left(\frac{m v^{2}}{2}\right)=-\gamma v^{2}-\frac{d}{d t}\left(\frac{k^{\prime} x^{2}}{2}\right)+\eta v= \\
=\frac{d}{d t}\left(\frac{m v^{2}}{2}+\frac{k^{\prime} x^{2}}{2}\right)=\frac{d E}{d t}=\eta v-\gamma v^{2}=\frac{d Q}{d t} .
\end{gathered}
$$

A Eq. (2-5) exprime a conservação de energia, mostrando que a potência que é injetada na partícula é proveniente de sua interação com o banho térmico. Ainda sobre a Eq. (2-5), observe que no regime estacionário para uma partícula $\left\langle\frac{d E}{d t}\right\rangle=0$, entretanto a corrente de calor é diferente de zero para o caso da cadeia linear por causa da interação entre as partículas das extremidades e suas primeiras vizinhas. Voltando para o caso da cadeia nota-se que o raciocínio aplicado a uma situação mais trivial, pode ser utilizado para determinar qual a corrente de calor transmitida pelos reservatórios térmicos às partículas das extremidades da cadeia. Como o interesse é determinar a expressão da corrente no regime estacionário, pode se escrever a energia injetada na partícula 1 a partir da Eq. (2-5) como:

$$
\langle J\rangle=\lim _{T \rightarrow \infty} \frac{\int_{0}^{T}\left\langle\left(\eta_{1}(t) v_{1, N}(t)-\gamma v_{1, N}^{2}(t)\right)\right\rangle d t}{T}
$$

Os índices 1 e $\mathrm{N}$ na Eq. (2-6) ilustram que a expressão pode ser calculada tanto para a primeira partícula como para a última, visto que no regime estacionário a corrente que é injetada em uma extremidade do sistema deve ser idêntica a que é transmitida pela partícula N para o outro reservatório. 


\subsection{2 \\ Método II}

Para calcular o calor transmitido entre duas partículas vizinhas da cadeia faz-se a análise novamente de um problema mais simples, verificando-se então que a solução deste pode ser aplicada ao caso da cadeia harmônica. Considere a situação onde duas partículas, 1 e 2, estão ligadas por uma haste rígida de massa desprezível e deseja-se calcular a potência fornecida pela partícula 1 à partícula 2. A maneira mais usual de calcular-se esta quantidade é utilizando a seguinte expressão:

$$
P=F_{1 \rightarrow 2} v_{2}
$$

onde $F_{1 \rightarrow 2}$ representa a força que a partícula 1 faz sobre a 2 e $v_{2}$ a velocidade desta última. Por se tratar de uma haste inderfomável a Eq. (2-7) é escrita de forma equivalente como

$$
P=\frac{F_{1 \rightarrow 2} v_{2}-F_{2 \rightarrow 1} v_{1}}{2}
$$

pois $F_{2 \rightarrow 1}=-F_{1 \rightarrow 2}$ e $v_{1}=v_{2}$. Para o caso da cadeia harmônica os termos $F_{1 \rightarrow 2}$ e $F_{2 \rightarrow 1}$ presentes na Eq. (2-8) são substituídos pela interação harmônica, de maneira que a potência (corrente) transmitida entre duas partículas quaisquer neste caso é:

$$
J=\frac{k_{1}\left(x_{i}(t)-x_{i+1}(t)\right)\left(v_{i}(t)+v_{i+1}(t)\right)}{2} .
$$

Assim, no regime estacionário o fluxo de calor também pode ser escrito como:

$$
\langle J\rangle=\frac{1}{2 T} \lim _{T \rightarrow \infty} \int_{0}^{T}\left\langle\left(x_{i}(t)-x_{i+1}(t)\right)\left(v_{i}(t)+v_{i+1}(t)\right)\right\rangle d t,
$$

onde $i=1,2, \ldots, N-1$. As expressões dadas pelas Eqs. (2-6) e (2-10) são equivalentes visto que a o fluxo de calor é igual entre todos os elementos da cadeia no regime estacionário, de forma que na seção a seguir escolhe-se desenvolver a Eq. (2-6) em perda de generalidade.

\subsection{3}

\section{Calculando o fluxo de calor}


Existem diferentes maneiras de se calcular o calor injetado, por isso aqui listam-se os métodos mais usuais utilizados além claro, de descrever a técnica utilizada neste trabalho.

Uma forma de se calcular a equação 2-6 é, por exemplo, resolver a equação de Fokker-Planck para o sistema e, através dela, extrair a função de densidade de probabilidade associada às posições e momentos lineares das partículas do sistema, podendo dessa maneira calcular os valores médios associados $[24,25,27]$.

Outra forma eficiente de executar esta tarefa é utilizando a transformada de Fourier [25, 26, 36], de onde as equações de movimento mostradas em 2-2 tornam-se bem mais simples, podendo ser diagonalizadas e determinando desta maneira a função de Green que resolve o problema. O método empregado neste trabalho utiliza o conceito da transformada de Fourier-Laplace, que mostra-se tão eficiente ou mais quanto aos outros métodos, oferecendo também uma forma sistemática de calcular outras quantidades associadas ao sistema que possuem um grau de dificuldade maior. Como as passagens matemáticas são um tanto extensas neste trecho remete-se o(a) leitor(a) ao apêndice A, mostrando-se aqui apenas os principais resultados.

A forma do fluxo de calor que será desenvolvida é dada por

$$
\begin{aligned}
\langle J\rangle & =\lim _{z \rightarrow 0} z \int_{0}^{\infty} d t e^{-z t}\left\langle\left(\eta_{1}(t) v_{1}(t)-\gamma v_{1}^{2}(t)\right)\right\rangle \\
& =\lim _{z \rightarrow 0} \lim _{\epsilon \rightarrow 0} z \int_{0}^{\infty} d t \int_{-\infty}^{\infty} \frac{d q_{1}}{2 \pi} \int_{-\infty}^{\infty} \frac{d q_{2}}{2 \pi} e^{-\left[z-\left(i q_{1}+i q_{2}+2 \epsilon\right)\right] t} \times \\
& \times\left\langle\left(\tilde{\eta}_{1}\left(i q_{1}+\epsilon\right) \tilde{v}_{1}\left(i q_{2}+\epsilon\right)-\gamma \tilde{v}_{1}\left(i q_{1}+\epsilon\right) \tilde{v}_{1}\left(i q_{2}+\epsilon\right)\right)\right\rangle \\
& =\lim _{z \rightarrow 0} \lim _{\epsilon \rightarrow 0} \int_{-\infty}^{\infty} \frac{d q_{1}}{2 \pi} \int_{-\infty}^{\infty} \frac{d q_{2}}{2 \pi} \frac{z}{z-\left(i q_{1}+i q_{2}+2 \epsilon\right)} \times \\
& \times\left\langle\left(\tilde{\eta}_{1}\left(i q_{1}+\epsilon\right) \tilde{v}_{1}\left(i q_{2}+\epsilon\right)-\gamma \tilde{v}_{1}\left(i q_{1}+\epsilon\right) \tilde{v}_{1}\left(i q_{2}+\epsilon\right)\right)\right\rangle,
\end{aligned}
$$

onde $\tilde{v}_{1}\left(i q_{i}+\epsilon\right)$ e $\tilde{\eta}_{1}\left(i q_{i}+\epsilon\right)$ representam, respectivamente, as transformada de Fourier-Laplace da velocidade da partícula 1 e do ruído. Com o intuito de resolver (2-11) em termos das posições das partículas apenas é necessário voltar à Eq. (2-2) para encontrar função que descreve a posição da partícula 1. Nota-se que a Eq. (2-2) pode ser escrita em forma matricial: 


$$
\begin{aligned}
\widetilde{\mathcal{D}}(\mathrm{i} q+\varepsilon) \tilde{\boldsymbol{x}}(\mathrm{i} q+\varepsilon) & =\tilde{\boldsymbol{\eta}}(\mathrm{i} q+\varepsilon) \\
\mathcal{A} & \equiv \mathcal{D}^{-1} \\
\tilde{\boldsymbol{x}}(\mathrm{i} q+\varepsilon) & =\tilde{\mathcal{A}}(\mathrm{i} q+\varepsilon) \tilde{\boldsymbol{\eta}}(\mathrm{i} q+\varepsilon),
\end{aligned}
$$

onde $\mathcal{D}(\mathrm{i} q+\varepsilon)$ representa um operador ${ }^{1} N \times N, \boldsymbol{x}(\mathrm{i} q+\varepsilon)$ é o vetor que fornece as transformadas de Fourier-Laplace das posições de cada um dos sítios, $\boldsymbol{x}(\mathrm{i} q+\varepsilon) \equiv\left\{x_{1}(\mathrm{i} q+\varepsilon), \ldots, x_{N}(\mathrm{i} q+\varepsilon)\right\}$ e $\boldsymbol{\eta}(\mathrm{i} q+\varepsilon) \equiv$ $\left\{\eta_{1}(\mathrm{i} q+\varepsilon), 0, \ldots, 0, \eta_{N}(\mathrm{i} q+\varepsilon)\right\}$ representa o vetor coluna que descreve as flutuações inseridas no sistema devido aos reservatórios.

Assume-se no tratamento do problema, por simplicidade e sem perda de generalidade, que $x_{i}(0)=0$ e $v_{i}(0)=0$ para todo $i$. Pode se escrever o termo de velocidade da partícula 1 em função de sua posição ao se utilizar a seguinte propriedade da transformada de Fourier-Laplace:

$$
\tilde{v}(\mathrm{i} q+\varepsilon)=(\mathrm{i} q+\varepsilon) \tilde{x}(\mathrm{i} q+\varepsilon) .
$$

Com o auxílio da Eq. (2-15) a equação (2-11) pode ser reescrita como

$$
\begin{aligned}
\langle J\rangle & =\lim _{z \rightarrow 0} \lim _{\epsilon \rightarrow 0} \int_{-\infty}^{\infty} \frac{d q_{1}}{2 \pi} \int_{-\infty}^{\infty} \frac{d q_{2}}{2 \pi} \frac{z}{z-\left(i q_{1}+i q_{2}+2 \epsilon\right)} \times \\
& \times\left(i q_{2}+\epsilon\right)\left\langle\tilde{\eta}_{1}\left(i q_{1}+\epsilon\right) \tilde{x}_{1}\left(i q_{2}+\epsilon\right)\right\rangle- \\
& -\lim _{z \rightarrow 0} \lim _{\epsilon \rightarrow 0} \int_{-\infty}^{\infty} \frac{d q_{1}}{2 \pi} \int_{-\infty}^{\infty} \frac{d q_{2}}{2 \pi} \frac{z}{z-\left(i q_{1}+i q_{2}+2 \epsilon\right)} \times \\
& \times \gamma\left(i q_{1}+\epsilon\right)\left(i q_{2}+\epsilon\right)\left\langle\tilde{x}_{1}\left(i q_{1}+\epsilon\right) \tilde{x}_{1}\left(i q_{2}+\epsilon\right)\right\rangle
\end{aligned}
$$

Da equação 2-14, a posição da partícula no espaço recíproco $i$ é

$$
\tilde{x}_{i}(\mathrm{i} q+\varepsilon)=\sum_{j=1, N} \tilde{\mathcal{A}}_{i j}(\mathrm{i} q+\varepsilon) \tilde{\eta}_{j}(\mathrm{i} q+\varepsilon)
$$

e o ruído no espaço recíproco, $\tilde{\eta}$ — que continua sendo gaussiano — é dado por

$$
\left\langle\tilde{\eta}_{i}\left(\mathrm{i} q_{1}+\varepsilon\right) \tilde{\eta}_{j}\left(\mathrm{i} q_{2}+\varepsilon\right)\right\rangle=\frac{2 \gamma T_{i}}{\mathrm{i} q_{1}+\mathrm{i} q_{2}+2 \varepsilon} \delta_{i j} .
$$

Unindo as equações 2-17 e 2-18, finalmente pode se escrever a expressão

${ }^{1}$ Um exemplo das formas de ambos operadores, $\mathcal{D}(\mathrm{i} q+\varepsilon)$ e $\mathcal{A}(\mathrm{i} q+\varepsilon)$, se encontra no apêndice B. 
para o fluxo de calor como:

$$
\begin{aligned}
& \langle J\rangle=\lim _{z \rightarrow 0} \lim _{\varepsilon \rightarrow 0} 2 \gamma T_{1} \int_{-\infty}^{\infty} \int_{-\infty}^{\infty} \frac{d q_{1} d q_{2}}{4 \pi^{2}} \frac{z}{z-\left(i q_{1}+i q_{2}+2 \varepsilon\right)} \frac{\widetilde{\mathcal{A}}_{11}\left(\mathrm{i} q_{1}+\varepsilon\right)}{\left(i q_{1}+i q_{2}+2 \varepsilon\right)}+ \\
& -\lim _{z, \varepsilon \rightarrow 0} 2 \gamma^{2} T_{1} \int_{-\infty}^{\infty} \int_{-\infty}^{\infty} \frac{d q_{1} d q_{2}}{4 \pi^{2}} \frac{z\left(i q_{1}+\varepsilon\right)\left(i q_{2}+\varepsilon\right)}{z-\left(i q_{1}+i q_{2}+2 \varepsilon\right)} \frac{\widetilde{\mathcal{A}}_{11}\left(\mathrm{i} q_{1}+\varepsilon\right) \widetilde{\mathcal{A}}_{11}\left(\mathrm{i} q_{2}+\varepsilon\right)}{\left(i q_{1}+i q_{2}+2 \varepsilon\right)}+ \\
& -\lim _{z, \varepsilon \rightarrow 0} 2 \gamma^{2} T_{N} \int_{-\infty}^{\infty} \int_{-\infty}^{\infty} \frac{d q_{1} d q_{2}}{4 \pi^{2}} \frac{z\left(i q_{1}+\varepsilon\right)\left(i q_{2}+\varepsilon\right)}{z-\left(i q_{1}+i q_{2}+2 \varepsilon\right)} \frac{\widetilde{\mathcal{A}}_{1 N}\left(\mathrm{i} q_{1}+\varepsilon\right) \tilde{\mathcal{A}}_{1 N}\left(\mathrm{i} q_{2}+\varepsilon\right)}{\left(i q_{1}+i q_{2}+2 \varepsilon\right)}
\end{aligned}
$$

Integrando em $i q_{2}$ e tomando o limite que $z \rightarrow 0$, tem-se:

$$
\begin{array}{r}
\langle J\rangle=\lim _{\varepsilon \rightarrow 0} T_{1}\left(\frac{\gamma}{\pi} \int_{-\infty}^{\infty}(i q+\varepsilon) \widetilde{\mathcal{A}}_{11}(\mathrm{i} q+\varepsilon) d q\right. \\
\left.+\frac{\gamma^{2}}{\pi} \int_{-\infty}^{\infty}(i q+\varepsilon)^{2} \widetilde{\mathcal{A}}_{11}(\mathrm{i} q+\varepsilon) \widetilde{\mathcal{A}}_{11}(-\mathrm{i} q-\varepsilon) d q\right) \\
+\frac{\gamma^{2} T_{N}}{\pi} \int_{-\infty}^{\infty}(i q+\varepsilon)^{2} \widetilde{\mathcal{A}}_{1 N}(\mathrm{i} q+\varepsilon) \widetilde{\mathcal{A}}_{1 N}(-\mathrm{i} q-\varepsilon) d q .
\end{array}
$$

Visando escrever a Eq. (2-20) de uma maneira mais compacta, avalia-se esta expressão no caso em que ambos os reservatórios possuem a mesma temperatura, i.e., $T_{1}=T_{N}$. Quando esta condição de contorno é satisfeita o fluxo de calor médio que atravessa o sistema é nulo $\langle J\rangle$, visto que o sistema encontra-se em equilíbrio. Assumindo que tal condição seja satisfeita, a equação (2-20) é escrita como

$$
\begin{gathered}
\frac{\gamma}{\pi} \int_{-\infty}^{\infty}(i q+\varepsilon) \widetilde{\mathcal{A}}_{11}(\mathrm{i} q+\varepsilon) d q+\frac{\gamma^{2}}{\pi} \int_{-\infty}^{\infty}(i q+\varepsilon)^{2} \widetilde{\mathcal{A}}_{11}(\mathrm{i} q+\varepsilon) \widetilde{\mathcal{A}}_{11}(-\mathrm{i} q-\varepsilon) d q= \\
=-\frac{\gamma^{2}}{\pi} \int_{-\infty}^{\infty}(i q+\varepsilon)^{2} \widetilde{\mathcal{A}}_{1 N}(\mathrm{i} q+\varepsilon) \widetilde{\mathcal{A}}_{1 N}(-\mathrm{i} q-\varepsilon) d q .
\end{gathered}
$$

Nota-se que o lado esquerdo da Eq. (2-21) corresponde ao termo entre parênteses na equação (2-20), o que permite representar o fluxo de calor do 
caso linear de uma forma mais condensada dada por:

$$
\langle J\rangle_{\mathcal{L}}=\lim _{\varepsilon \rightarrow 0} \gamma^{2} \frac{\Delta T}{\pi} \int_{-\infty}^{\infty}(\mathrm{i} q+\varepsilon)^{2} \tilde{\mathcal{A}}_{1 N}(\mathrm{i} q+\varepsilon) \tilde{\mathcal{A}}_{1 N}(-\mathrm{i} q-\varepsilon) d q
$$

com $\Delta T \equiv T_{N}-T_{1}$. Para tornar o entendimento da presente técnica algo mais tangível, na próxima seção será apresentado um caso particular de sua aplicação, além de analisar-se o que acontece com o comportamento do fluxo de calor em uma cadeia harmônica conforme aumenta-se seu tamanho.

\section{5}

\section{Exemplos numéricos e discussão dos resultados}

Para ter uma noção da física que está envolvida no problema e do perfil das funções a serem integradas, ilustra-se um caso bem simples onde a cadeia térmica é composta por apenas dois blocos. Neste caso a função que surge no integrando da Eq. (2-22) é escrita como:

$$
\widetilde{\mathcal{A}}_{1 N}(\mathrm{i} q+\varepsilon)=\frac{k_{1}}{-k_{1}^{2}+\left[m(\mathrm{i} q+\varepsilon)^{2}+\gamma(\mathrm{i} q+\varepsilon)+k^{\prime}+k_{1}\right]} .
$$

Uma formulação mais geral para a obtenção da função $\widetilde{\mathcal{A}}_{1 N}(\mathrm{i} q+\varepsilon)$ encontra-se no apêndice C. Em sistemas harmônicos lineares sabe-se que o fluxo de calor é proporcional à diferença de temperaturas entre os reservatórios $[24,27]$, i.e.:

$$
\langle J\rangle=\lim _{\varepsilon \rightarrow 0} \gamma^{2} \frac{\Delta T}{\pi} \int_{-\infty}^{\infty}(\mathrm{i} q+\varepsilon)^{2} \widetilde{\mathcal{A}}_{1 N}(\mathrm{i} q+\varepsilon) \widetilde{\mathcal{A}}_{1 N}(-\mathrm{i} q-\varepsilon) d q=\Lambda \Delta T,
$$

onde $\Lambda$ é a constante de proporcionalidade denominada condutância, que mede a energia que flui ao longo do sistema.

Uma vez que $\widetilde{\mathcal{A}}_{1 N}(\mathrm{i} q+\varepsilon)$ é determinada, o próximo passo é integrar esta função no plano complexo, onde escolhe-se como caminho de integração um semi-círculo na parte superior do plano complexo (cf. Fig. 2.2). Tomando-se os parâmetros da dinâmica do problema com valores iguais a 1 , exceto por $k^{\prime}$, que vale 0.5 , e $k$ que é igual a 0 , visto que o sistema não possui elementos em seu volume, o fluxo de calor no sistema é igual a:

$$
\langle J\rangle=\Lambda \Delta T=-0.2 \Delta T=-0.2\left(T_{N}-T_{1}\right) .
$$


Um ponto que vale a pena ser levantado aqui é que todas as integrações realizadas para cadeias com $N>2$ acabam se tornando bem simples de serem analisadas, pois os pólos que surgem são provenientes das raízes de um polinômio, que nada mais é que o determinante da matriz da dinâmica (cf. Eq. (C-2)).

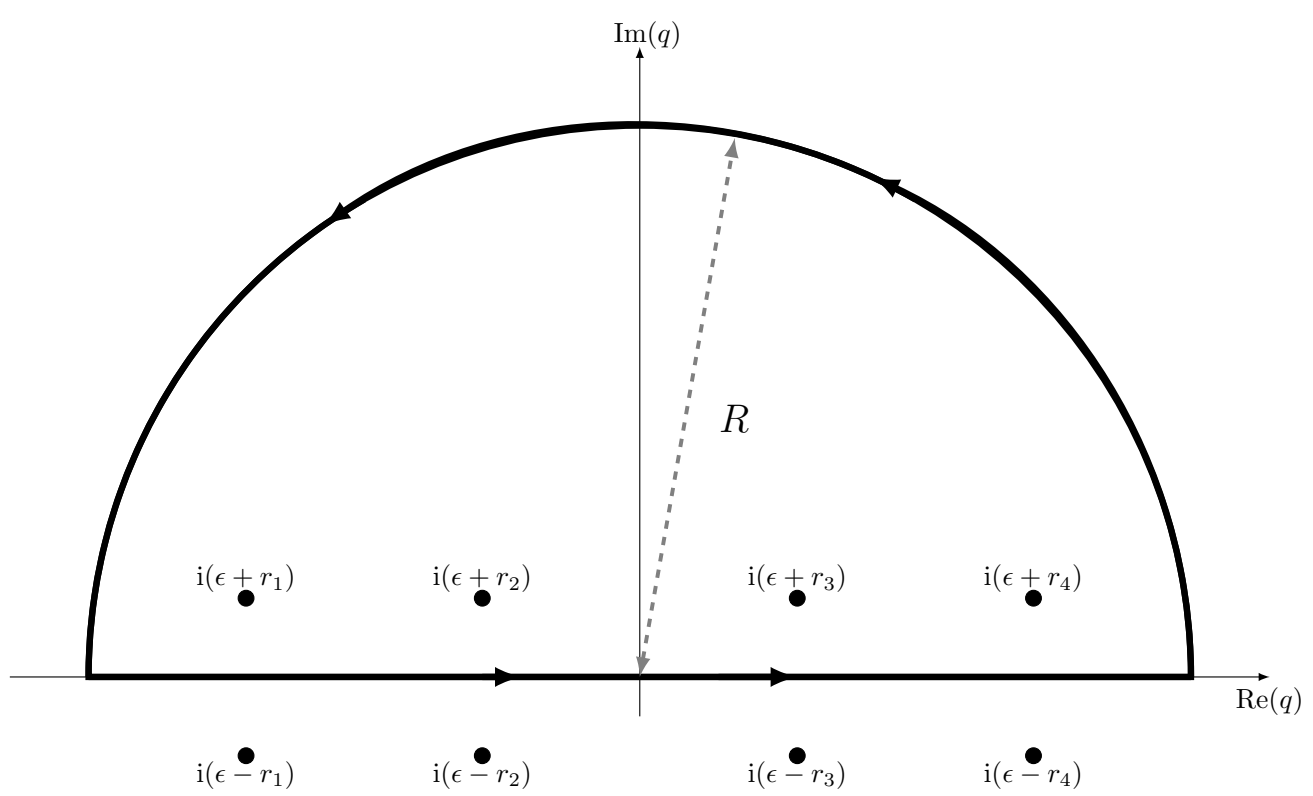

Figura 2.2: Disposição dos pólos da dinâmica do problema do exemplo em que $N=2$. Os termos $i\left(\epsilon+r_{1}\right), i\left(\epsilon+r_{2}\right), i\left(\epsilon+r_{3}\right)$ e $i\left(\epsilon+r_{4}\right)$ representam os pólos da função $\widetilde{\mathcal{A}}_{1 N}(\mathrm{i} q+\varepsilon)$, enquanto os termos $i\left(\epsilon-r_{1}\right), i\left(\epsilon-r_{2}\right), i\left(\epsilon-r_{3}\right)$ e $i\left(\epsilon-r_{4}\right)$ representam os pólos não integráveis de $\widetilde{\mathcal{A}}_{1 N}(-\mathrm{i} q-\varepsilon)$.

É razoável se imaginar que a condutância de uma cadeia seja uma grandeza que dependa de seu tamanho. Para entender esta última justificativa suponha que no sistema em questão a lei de Fourier seja válida, de forma que o fluxo de calor pode ser esrito como:

$$
\langle J\rangle=-\kappa \nabla T=-\Lambda \Delta T
$$

Considere agora que a distância típica entre os elementos da cadeia seja igual a $d$. Se a cadeia possui um tamanho igual a $L$ e possui $N$ elementos, sendo $N \gg 1$, é simples notar que: 


$$
L=N d \text {. }
$$

Assim, nota-se que a Eq. (2-26) pode ser escrita como,

$$
\langle J\rangle=-\kappa \frac{\Delta T}{d N}
$$

de onde pode se inferir que

$$
\Lambda=\kappa / N d
$$

ou seja, em um sistema em que a lei de Fourier seja válida a condutância deste deve obedecer a seguinte relação: $\Lambda \propto N^{-1} \propto L^{-1}$. Uma outra alternativa para verificar que a condutância decresce com o tamanho do sistema é dada pelo seguinte raciocínio: imagine que o limite termodinâmico seja tomado em um sistema onde a lei de Fourier é válida, de forma que a corrente de calor ao longo do sistema deve ir a zero neste limite, visto que a resistência térmica do sistema seria infinita. Como consequência, a condutância do sistema que obedece a lei de Fourier deve diminuir de forma proporcional ao aumento do tamanho do sistema.

Para verificar o comportamento da condutância em um modelo harmônico, mostra-se ao longo dessa seção o resultado do fluxo de calor para cadeias de múltiplos $N^{\prime} s$. A partir da Eq. (2-24) é possível construir a figura 2.3, onde mostra-se como a condutância se comporta conforme aumenta-se o tamanho da cadeia. 


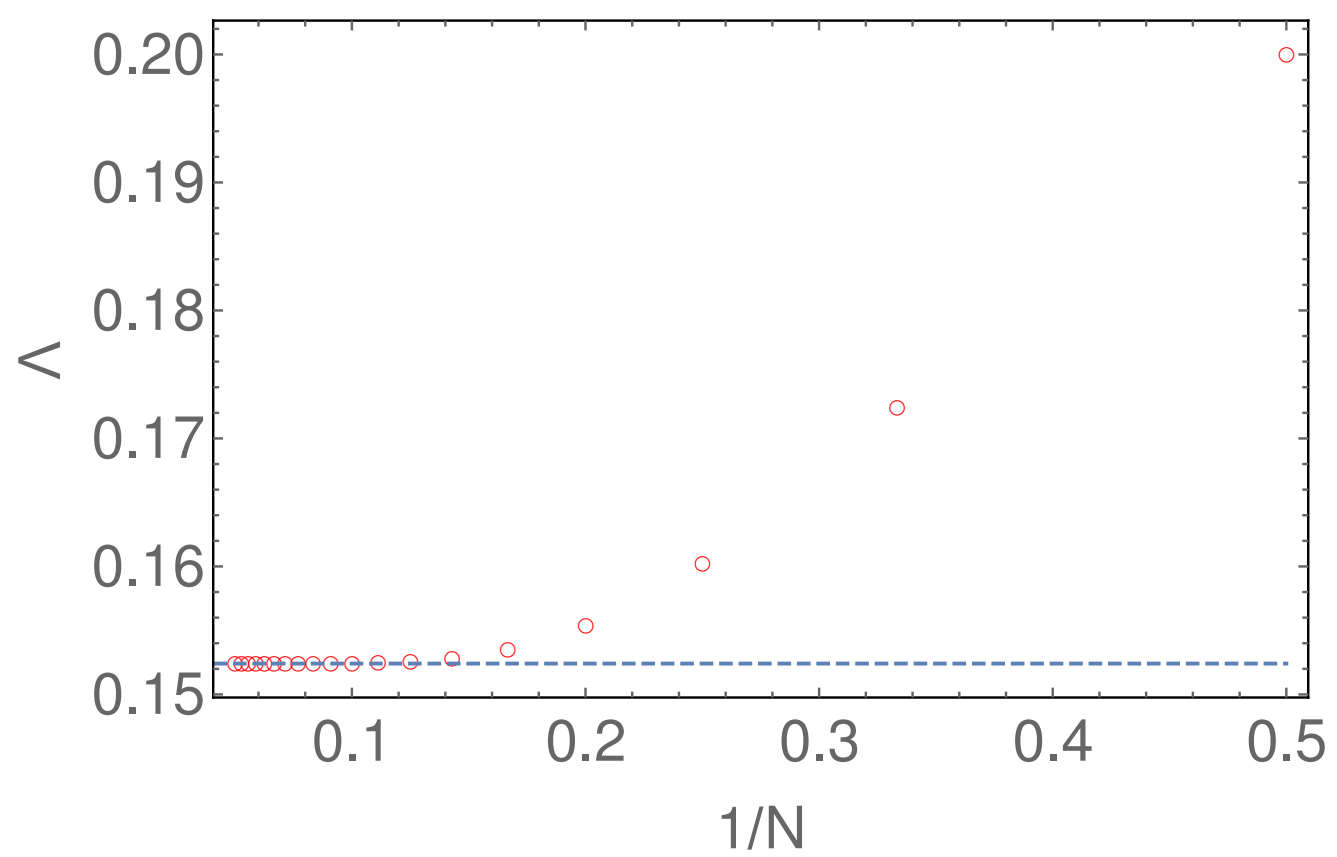

Figura 2.3: Gráfico descrevendo a condutância versus $\frac{1}{N}$.

Como pode se observar na figura 2.3, a condutância converge para um valor constante, contrariando o comportamento físico ideal. O fato de a condutância térmica assumir um valor finito é uma característica inerente aos modelos unidimensionais que possuem apenas interações harmônicas [24-27, 57], sendo então o transporte de energia que ocorre nestes modelos caracterizado como balístico.

Em sistemas que possuem apenas interações harmônicas em suas equações de movimento nota-se que quando $N \longrightarrow \infty$ a condutividade, $\kappa$, diverge linearmente com o tamanho do sistema, informação que pode ser expressa da seguinte forma:

$$
\kappa \propto N \propto L .
$$

Este resultado mostra que o gradiente de temperatura ao longo da cadeia é basicamente nulo, devido ao valor finito que o fluxo de energia assume enquanto o tamanho do sistema aumenta. Esta saturação do fluxo de calor que caracteriza o regime balístico, pode ser entendida de forma bem simples: sistemas harmônicos puros possuem modos normais que são separáveis e consequentemente os seus fônons não interagem entre si.

A simplicidade do modelo linear não é capaz de incorporar o espalhamento de fônons que ocorre em um sistema físico real. Efeitos como 
de superfície, impurezas e imperfeições no material, que atuam como centros espalhadores, devem ser inseridos no sistema de alguma maneira para que a descrição do problema se aproxime ao máximo da situação real. Um exemplo mais cotidiano da necessidade da inserção de não-linearidades na interações de um cristal é ilustrado pelo fenômeno da dilatação térmica (cf. Fig. 2.4), o qual ocorre justamente devido à interações que vão além da aproximação harmônica [31].

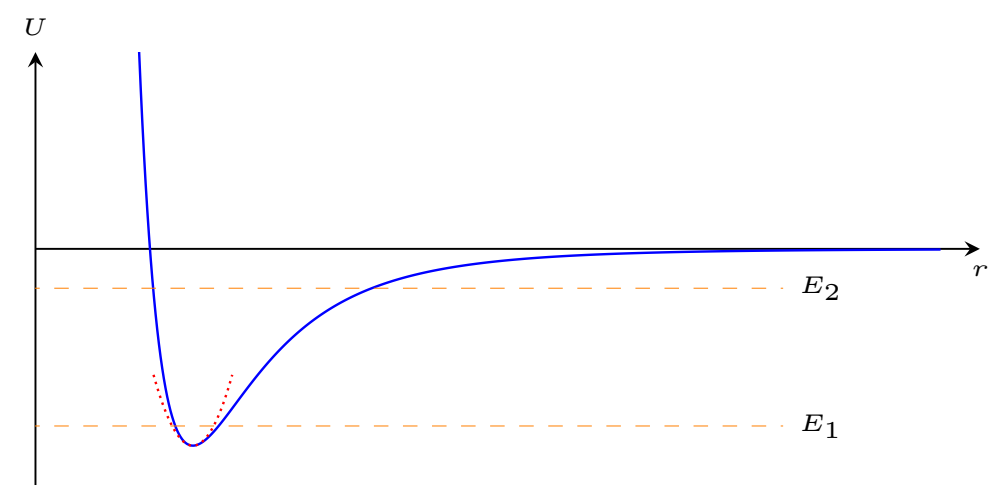

Figura 2.4: A figura ilustra qualitativamente o potencial elástico de um cristal em duas situações: i) região onde a expansão harmônica é razoável e a energia do sistema é dada por $E_{1}$; ii) região onde os efeitos das não linearidades devem ser levados em consideração com energia $E_{2}$.

Os efeitos citados podem ser incluídos em um sistema, por exemplo, através de interações anarmônicas entre os elementos da cadeia e/ou com o próprio substrato [32, 47, 49]. Outras maneiras de provocar o espalhamento dos fônons ao longo da cadeia consistem, por exemplo, em utilizar uma cadeia que possua dispersão de massa [25, 36, 29] ou uma interação de banhos térmicos com o volume do sistema [58-61]. Embora em alguns casos dentre os citados seja possível encontrar uma condutividade térmica finita $[47,58,59,61]$, os demais exemplos mostram que esta grandeza é função de $L$, mais precisamente, $\kappa \propto L^{\alpha} \propto N^{\alpha}$, com $\alpha>0$, ou seja, o problema da divergência da condutividade permanece presente apesar das alterações no modelo.

A anomalia apresentada na condução de calor deve ser refletida de alguma maneira no perfil de temperatura da cadeia. O gradiente quasi-nulo de temperatura pode ser observado quando se compara as temperaturas, estas definidas como a energia cinética média de cada partícula, ao longo do volume do sistema. Embora o comportamento das temperaturas ao longo do 
volume pareça ser bem entendido, pelo menos qualitativamente, existe um fator pouco explorado associado a este perfil: a relação entre os pârametros de acoplamento que regem a dinâmica do sistema. Qual a mudança sofrida por grandezas macroscópicas e estatísticas do sistema com a alteração destes parâmetros? A resposta para esta questão e outras vindouras serão exploradas nas próximas seções. 


\section{3}

\section{O papel das correlações entre as velocidades nos perfis de temperatura do sistema}

\section{1}

\section{Possíveis perfis de temperatura de uma cadeia harmônica linear}

O estudo da distribuição de temperaturas, ao longo da cadeia harmônica linear permite analisar, através da energia cinética média associada a cada sítio, se o modelo utilizado é capaz ou não de descrever um comportamento de condução de calor condizente com a lei de Fourier. Partindo do teorema da equipartição de energia, pode se escrever a temperatura de cada elemento da cadeia como

$$
\mathcal{T}_{i}=m\left\langle v_{i}^{2}\right\rangle
$$

onde supôs-se que a constante de Boltzmann é igual a um. Podemos reescrever a Eq. (3-1) através da técnica ilustrada no capítulo 1. Aplicando a transformada de Fourier-Laplace em (3-1) tem-se

$$
\mathcal{T}_{i}=m \lim _{z \rightarrow 0, \varepsilon \rightarrow 0} \int_{-\infty}^{+\infty} \frac{d q_{1}}{2 \pi} \frac{d q_{2}}{2 \pi} \frac{z\left\langle\tilde{v}_{i}\left(\mathrm{i} q_{1}+\varepsilon\right) \tilde{v}_{i}\left(\mathrm{i} q_{2}+\varepsilon\right)\right\rangle}{z-\left(\mathrm{i} q_{1}+\mathrm{i} q_{2}+2 \varepsilon\right)} .
$$

Com o auxílio das Eqs. (2-17) e (2-18), pode se chegar facilmente à expressão para a temperatura em cada sítio, dada por:

$$
\mathcal{T}_{i}=\frac{m \gamma}{\pi} \sum_{j=1, N} T_{j} \int_{-\infty}^{+\infty}(\mathrm{i} q+\varepsilon)(-\mathrm{i} q-\varepsilon) \tilde{\mathcal{A}}_{i j}(\mathrm{i} q+\varepsilon) \tilde{\mathcal{A}}_{i j}(-\mathrm{i} q-\varepsilon) d q
$$

A discussão sobre o comportamento das temperaturas em cadeias harmônicas homogêneas já foi introduzida previamente [25, 27], onde mostrou-se detalhadamente que o perfil de temperaturas de uma cadeia onde os elementos do volume não possuem acoplamento com o substrato $(k=0)$. Esta escolha na hamiltoniana do sistema acaba se refletindo no perfil de temperaturas, que de forma muito contra-intuitiva mostra que as temperaturas das partículas mais próximas ao reservatório com temperatura $T_{1}$ possuem uma temperatura maior que a temperatura média entre os 
Capítulo 3. O papel das correlações entre as velocidades nos perfis de temperatura do sistema

reservatórios, enquanto que as partículas vizinhas ao banho térmico com temperatura $T_{N}$ possuem uma temperatura menor do que a média das temperatura entre os reservatórios! O perfil de temperatura associado a estes modelos encontra-se ilustrado na figura 3.1(a), onde pode notar-se que as únicas partículas às quais este comportamento não se aplica são as partículas 1 e $N$.

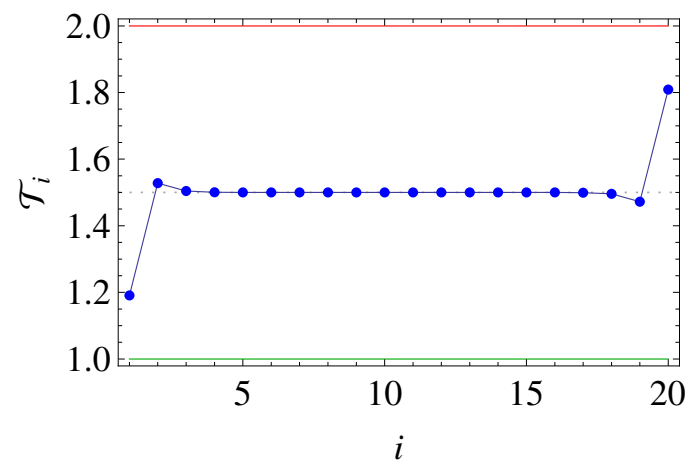

$3.1(\mathrm{a}): k=0$

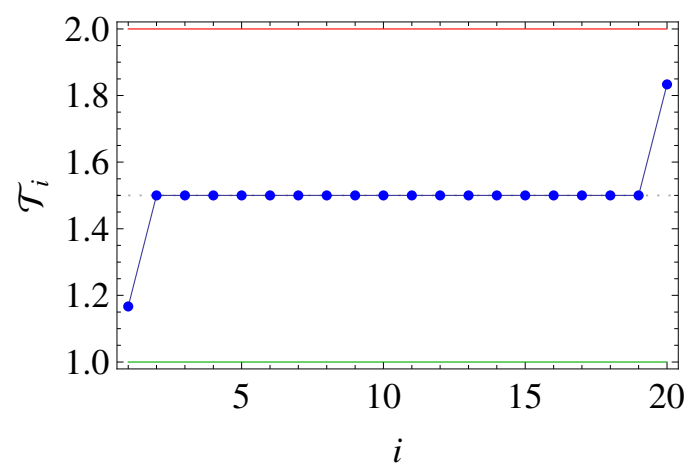

$3.1(\mathrm{~b}): k=\frac{1}{2}$

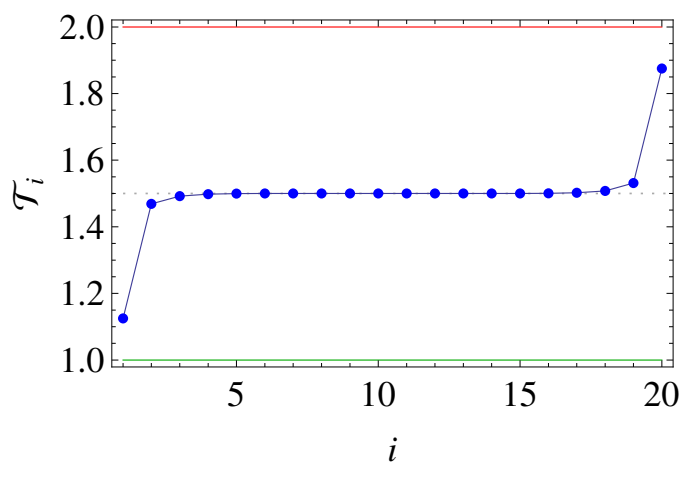

$3.1(\mathrm{c}): k=1$

Figura 3.1: As figuras (a), (b), e (c) mostram os possíveis perfis de temperatura em uma cadeia linear. Aqui tomou-se $k_{1}=k^{\prime}=\gamma=m=1$ e uma cadeia com $\mathrm{N}=20$. As temperaturas dos reservatórios térmicos correspondem as linhas verde $\left(T_{1}=1\right)$ e vermelha $\left(T_{N}=2\right)$.

Um fato é que este perfil de temperaturas não representa universalmente os modelos harmônicos de cadeia linear. Pode-se suavizar este perfil de temperatura um tanto incomum ao se introduzir o acoplamento de ancoragem no volume do sistema nas equações de movimento do sistema. Adotando esta mudança, nota-se que as cúspides próximas às extremidades vão se tornando mais suaves conforme o parâmetro de ancoragem de volume aumenta. As cúspides são eliminadas do perfil de temperaturas quando os acoplamentos atingem o limiar [62]: 


$$
k_{\text {crit }}=\frac{k^{\prime}+k_{1}}{4},\left(k^{\prime}=k_{1}\right)
$$

Quando a condição referente à Eq. (3-4), obtida de análise numérica, é imposta aos parâmetros de ancoragem da cadeia observa-se que a temperatura de todos os elementos associado ao volume do sistema é igual à $\frac{T_{1}+T_{N}}{2}$. Nota-se que o perfil do platô de temperaturas, mostrado na figura 3.1(b), dá a falsa impressão de que o volume do sistema encontra-se em equilíbrio térmico, como se as ondas formadas pela composição dos fônons da rede estivessem restritas a se propagarem apenas na região onde as temperaturas dos sítios são iguais, sem nenhuma influência das partículas das extremidades e, por conseguinte, dos reservatórios. Isto seria verdade caso a ancoragem $k^{\prime}$ das bordas fosse infinita; neste caso ter-se-ia um sistema que definimos aqui como caixa de fônons, onde o volume do sistema estaria livre de influência dos reservatórios devido ao isolamento das partículas 1 e $N$, ou caso o acoplamento de volume, $k$, fosse muito maior que os demais acoplamentos da cadeia, de forma que o volume se comportasse como sendo um sistema isolado com uma temperatura idêntica à média entre as temperaturas dos reservatórios, praticamente insensível às interações com as partículas das extremidades. A figura 3.2 mostra como a temperatura da partícula 2 varia conforme aumenta-se o valor de $k$, de onde é possível verificar que o mínimo apresentado pelas curvas é consistente com o que se espera do sistema no limite $k \rightarrow \infty$, i.e., a temperatura desta partícula só pode decrescer até um determinado ponto, tornando a aumentar posteriormente e por fim, quando o sistema encontra-se praticamente desacoplado dos reservatórios, apresentar uma temperatura que é igual a média entre as temperaturas dos reservatórios.

O surgimento desta pseudo caixa de fônons será discutido posteriormente quando une-se o estudo sobre os cumulantes do fluxo de calor ao platô de temperaturas que o sistema apresenta nesta configuração e as funções de correlação pertinentes ao estudo da cadeia harmônica linear. Por hora continua-se descrevendo como as mudanças na ancoragem alteram o perfil de temperatura do sistema.

Abandonando a restrição feita em (3-4) sobre a igualdade entre o acoplamento interpartículas e a ancoragem de borda, existem outras relações mais gerais que levem à mudança entre regimes de temperatura em uma cadeia harmônica linear? Será mostrado que, dependendo das relações existentes entre o acoplamento entre as partículas e as ancoragens do modelo, o sistema pode apresentar não apenas uma, mas outras transições de regime de temperaturas. Em vez de mudar a ancoragem do volume, qual a resposta dada pelo sistema, 


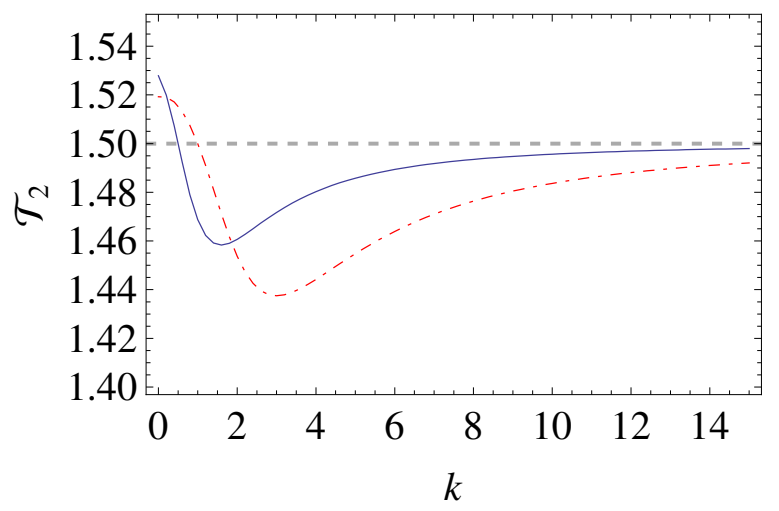

Figura 3.2: Temperatura da partícula $2, \mathcal{T}_{2}$, vs ancoragem de volume, $k$. com $m=1, \gamma=1$. A linha azul cheia corresponde aos parâmetros $k^{\prime}=k_{1}=1$ e a linha vermelha tracejada-pontilhada está associada a $k^{\prime}=k_{1}=2$. Os valores críticos de $k$ são, $k_{\text {crit }}=1 / 2$ e $k_{\text {crit }}=1$, respectivamente, de acordo com Eq. (3-4). Para valores de $\mathrm{k}$ tal que $k \rightarrow \infty$, a cadeia se transforma em um fio rígido e a temperatura do volume naturalmente tende a $3 / 2$.

em termos de perfil de temperatura, caso se varie a ancoragem de borda, $k^{\prime}$ ?

Suponha que na cadeia altere-se $k^{\prime}$ suavemente a partir de zero, admitindo que $k \neq 0$. Observa-se que para uma determinada faixa de valores de $k^{\prime}$ o sistema passa a apresentar um comportamento, no que diz respeito à distribuição de temperaturas das partículas do volume, oposto ao descrito por RLL. Os elementos da cadeia mais próximos ao reservatório frio possuem temperaturas menores que $\frac{T_{1}+T_{N}}{2}$, já os sítios mais próximos do reservatório quente possuem suas temperaturas maiores que $\frac{T_{1}+T_{N}}{2}$. O aspecto da temperatura do sistema pode ser visualizado na figura 3.1(c), onde pode notar-se que as cúspides próximas às extremidades da cadeia foram substituídas por curvas com um perfil bem mais suave. Ao aumentar-se $k^{\prime}$ continuamente, nota-se que as temperaturas das partículas 2 e $N-1$ começam a se aproximar da média de temperatura entre os reservatórios, até atingir a configuração onde o volume da cadeia atinge a temperatura $\frac{T_{1}+T_{N}}{2}$ e a ancoragem de borda é dada pela Eq. (3-5), (cf. 3.1(b)).

$$
k_{\text {crit }}^{\prime}=\frac{k}{2}+\frac{\sqrt{k^{2}+4 k k_{1}}}{2},
$$

a origem da Eq. (3-5) será justificada a posteriori juntamente com a discussão dos cumulantes do fluxo de calor. A Eq. (3-5) representa a linha vermelha na figura 3.3, delimitando as regiões em que o sistema apresenta um perfil de temperaturas suave e o perfil do modelo RLL.

Ao observar-se a figura 3.3, nota-se que para uma dada região onde 
Capítulo 3. O papel das correlações entre as velocidades nos perfis de temperatura do sistema

$k^{\prime}>k_{\text {crit }}^{\prime}$ as cúspides voltam a surgir no sistema, mostrando que a presença da ancoragem de volume é um fator necessário mas não suficiente para que o sistema não exiba tal característica em seu perfil de temperatura. O perfil semelhante ao modelo RLL é apresentado para todos os valores de $k^{\prime}$ inferiores a $[62]$

$$
k_{c r i t_{2}}^{\prime}=k+k_{1}+\frac{\gamma^{2}}{m}
$$

onde a Eq. (3-6), representada pela linha azul na figura 3.3, é obtida heuristicamente e exprime a condição para que o volume do sistema encontre-se termalizado pela segunda vez. Após o sistema atingir o segundo platô, tem-se novamente o perfil de temperaturas bem suave próximo às extremidades, marcando assim mais uma mudança de comportamento das partículas mediante à mudança de $k^{\prime}$. A partir de então, para um crescimento contínuo do acoplamento das extremidades, nota-se que tal mudança tende a isolar as partículas 1 e $N$ do restante da cadeia, de forma que o volume do sistema passa a interagir praticamente com si próprio, criando assim a caixa de fônons já descrita anteriormente. As mudanças entre os diferentes regimes de temperaturas podem ser visualizadas na figura 3.3.

Aparentemente, o fato de a cadeia apresentar ou não cúspides no

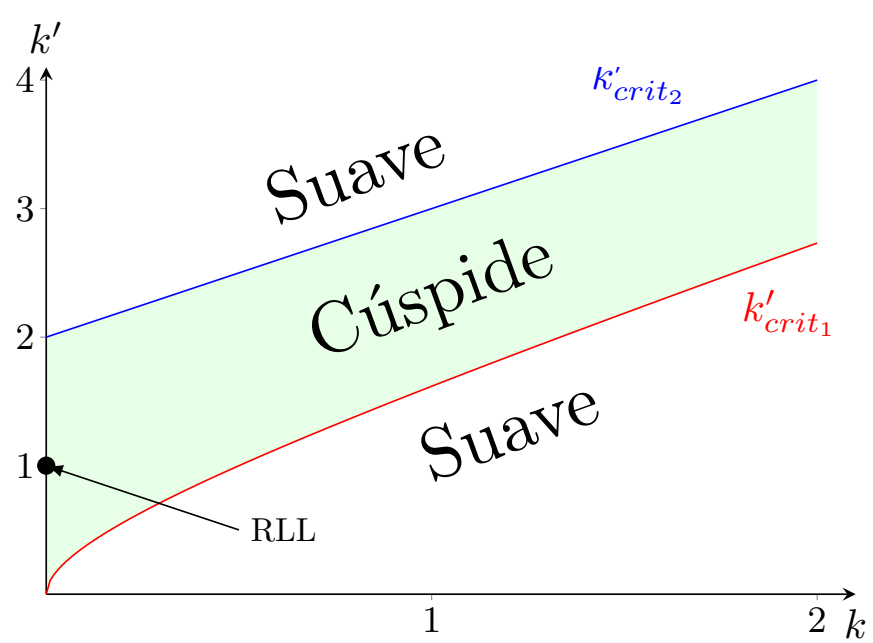

Figura 3.3: Regiões do espaço onde a cadeia apresenta perfil de temperaturas do modelo RLL (cúspides) e do modelo estudado. As linhas vermelha e azul representam, respectivamente, a $1^{\mathrm{a}}$ e $2^{\mathrm{a}}$ transição entre os regimes de temperatura. Ilustra-se também na figura um ponto correspondente ao regime RLL, onde $k^{\prime}=k_{1}=1$ e $k=0$. 
Capítulo 3. O papel das correlações entre as velocidades nos perfis de temperatura do sistema

perfil de temperatura sugere que tais respostas do sistema estão associadas a ajustes mecânicos do problema, mas as seções seguintes mostrarão a física por trás destes fenômenos. A primeira transição entre regimes aqui apresentada descreve mais do que apenas uma escolha conveniente dos parâmetros do problema. Nas próximas seções será mostrado que ela possui influência nos cumulantes da distribuição de $J$, bem como um papel importante na distribuição dos modos vibração da rede.

\section{2}

\section{Funções de correlação e modos de vibração em uma cadeia}

A mudança nos perfis de temperatura da cadeia harmônica linear devido a variações dos parâmetros associados à dinâmica do sistema sugerem que o fluxo de calor que o atravessa é afetado por tais ajustes. O ponto importante é: de que maneira um aumento contínuo da ancoragem das extremidades influencia na transferência de energia ao longo da cadeia? Para responder esta pergunta deve-se voltar à Eq. (2-22) e analisar-se o limite em que a cadeia possui um número de elementos $N \rightarrow \infty$.

Neste regime, pode se mostrar que (ver apêndice C) no limite termodinâmico (2-22) converte-se em:

$$
\langle J\rangle_{c}=\frac{\gamma k_{1}^{2}}{m \Theta^{2}}\left(\Pi-\sqrt{\Pi^{2}-\Theta^{2}}\right) \Delta T,
$$

onde,

$\Theta=\frac{2 k_{1} \gamma^{2}}{m}+2 k_{1}\left(k_{1}+k-k^{\prime}\right), \quad \Pi=\frac{\left(2 k_{1}+k\right) \gamma^{2}}{m}+\left(k_{1}+k\right)^{2}+\left(k_{1}-k^{\prime}\right)^{2}-2 k^{\prime} k$.

Pode-se extremar a Eq. (3-7), por exemplo, com respeito a constante de ancoragem das bordas, $k^{\prime}$, de forma que pode se estabelecer uma relação entre os parâmetros da dinâmica do problema e tornar o fluxo transmitido o máximo possível. Isolando $k^{\prime}$ das demais variáveis na equação associada à derivada do fluxo de calor com respeito a $k^{\prime}$, encontra-se a equação (3-5) mostrada na seção anterior.

O fato de que quando $k^{\prime}$ é dado pela Eq. (3-5) a energia transportada é maximizada ao longo do sistema é uma das maneiras de se distinguir entre a primeira e a segunda mudança de regimes de temperatura que uma cadeia harmônica linear pode sofrer. Outra conclusão que é possível tirar da análise da Eq. (3-7) e das figuras 3.4, 3.5 é que a ancoragem de borda está associada à distribuição de probabilidades do fluxo de calor do sistema, de forma que outros cumulantes do fluxo de calor devem sofrer mudanças devido a variações dos valores de $k^{\prime}$. As condições de contorno nas bordas do sistema são fundamentais 
Capítulo 3. O papel das correlações entre as velocidades nos perfis de temperatura do sistema

no que diz respeito ao transporte de calor, tendo este fato sido observado em cadeias de 1, 2 e 3 dimensões [25, 26, 63].

Para compreender o que acontece com os cumulantes da distribuição do fluxo de calor, verifica-se o que acontece com os cumulantes variância, obliquidade e curtose, que oferecem resultados suficientes para descrever o comportamento dos demais cumulantes. Todas estas grandezas foram computadas analiticamente e o tanto seu cálculo explícito quanto comparações com simulações se encontram no apêndice F.

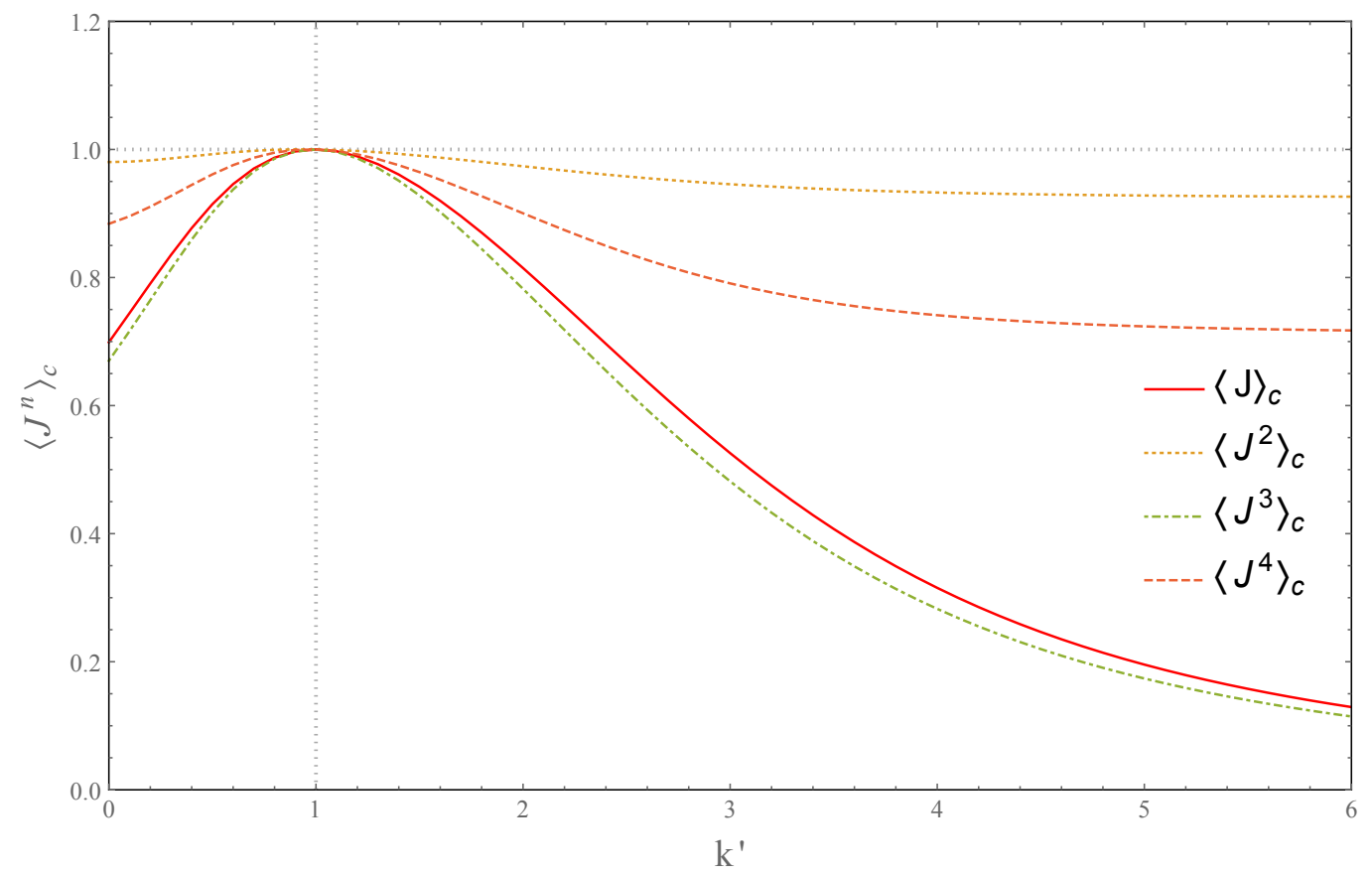

Figura 3.4: Cumulantes normalizados versus $k^{\prime}$. Nota-se que todos os cumulantes atingem o extremo justamente no ponto em que o sistema atinge a primeira mudança de regime.

Como pode se notar nas figuras 3.4 e 3.5, todos os cumulantes e momentos do fluxo de calor atingem um extremo no ponto em que ocorre a primeira mudança para o regime RLL, o que acaba por acentuar a diferença entre as duas transições de regimes de temperatura exibidas pela cadeia. A explicação para o porquê dos cumulantes ímpares da distribuição irem a zero está associada à medida matemática que estas grandezas representam para o sistema, pois estes cumulantes medem assimetrias associadas à distribuição de probabilidades do fluxo de calor, ou seja, se $\mathcal{T}_{i}=T_{N}=T_{1}$ tem-se que $\left\langle J^{2 n+1}\right\rangle_{c}=0$. Como para $k^{\prime}$ infinitamente grande tem-se o volume do sistema isolado dos reservatórios e termalizado, fica claro que qualquer cumulante ímpar associada a $J$ é zero. Já os cumulantes pares desta distribuição acabam por encontrar um valor de saturação, o que é simples de se visualizar, visto que no equilíbrio o valor médio 

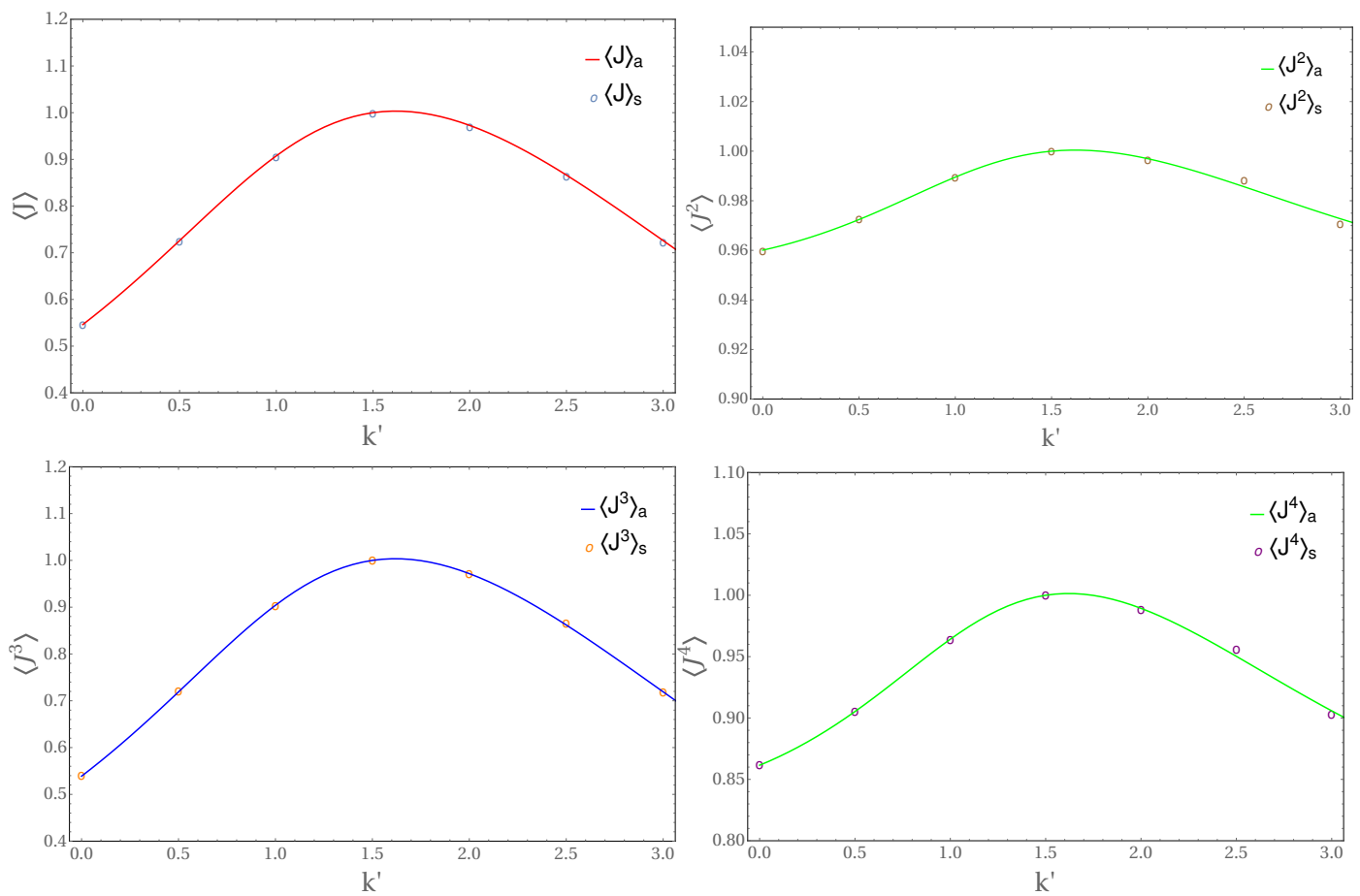

Figura 3.5: Comparação dos resultados numéricos e analíticos para os primeiros quatro momentos dos fluxo de calor. Os gráficos ilustram os momentos normalizados pelos seu respectivos valores máximos versus a ancoragem de borda, onde usou-se $m=1, \gamma=1$ e $k=1, k_{1}=1$. Os painéis superiores esquerdo e direito ilustram $\langle J\rangle$ e $\left\langle J^{2}\right\rangle$, respectivamente, enquanto os painéis inferiores e direito mostram $\left\langle J^{3}\right\rangle \mathrm{e}\left\langle J^{4}\right\rangle$. As linhas cheias representam a curva analítica e as circunferências os pontos obtidos através da simulação numérica. Para este conjunto de parâmetros os momentos atingem o seu máximo para $k_{\text {crit }}^{\prime} \approx 1.6180$.

de qualquer grandeza cinemática pode ser calculado utilizando a distribuição de Boltzmann-Gibbs.

O fato dos cumulantes atingirem um extremo no mesmo ponto remete a um possível paralelo ao estudo de transições de fase [5, 64, 65], onde a mudança de caracteríticas macroscópicas associadas a fases do sistema coincidem exatamente com o colapso dos cumulantes do fluxo de calor. Em uma transição de fase é sabido também que o comprimento de correlação entre os elementos do sistema torna-se infinito, característica que será estudada nesta seção para descobrir se, realmente, o fenômeno da mudança de regimes corresponde a uma transição de fase. Como associa-se as "fases" do sistema ao perfil de temperatura é razoável sugerir de um ponto de vista fenomenológico que a função de correlação entre as partículas seja dada por:

$$
C_{i, i+l}=\frac{\left\langle T_{i} T_{i+l}\right\rangle-\left\langle T_{i}\right\rangle\left\langle T_{i+l}\right\rangle}{\sqrt{\left\langle T_{i}^{2}\right\rangle-\left\langle T_{i}\right\rangle^{2}} \sqrt{\left\langle T_{i+l}^{2}\right\rangle-\left\langle T_{i+l}\right\rangle^{2}}},
$$


Capítulo 3. O papel das correlações entre as velocidades nos perfis de temperatura do sistema

de forma que

$$
C_{v^{2}}=A(\delta) \exp [-\delta / \xi]
$$

onde $\xi$ representa o comprimento de correlação entre as partículas, $\delta$ a distância entre os elementos vizinhos e $A(\delta)$ a amplitude de correlação.

Observa-se que a analogia da transição de fase torna-se um pouco mais clara quando analisa-se o platô de temperaturas, e interpreta-se tal configuração como sendo uma espécie de "fase ordenada" do sistema, justamente onde ocorre a mudança de regimes. Esta última afirmativa pode ser entendida com mais clareza ao supor que o platô é o estado crítico separando a configuração suave,

$$
\sum_{i=2}^{N / 2} \mathcal{T}_{i}-\mathcal{T}_{N-i+1}<0
$$

da configuração em que o sistema apresenta a cúspide,

$$
\sum_{i=2}^{N / 2} \mathcal{T}_{i}-\mathcal{T}_{N-i+1}>0 .
$$

Como a função de correlação encontra-se definida, o próximo passo é verificar se há transição de fase checando se o comprimento de correlação para diversos valores de $k^{\prime}$ é próximo à primeira mudança de comportamento, obedecendo à seguinte equação:

$$
\xi \sim\left|\Delta k^{\prime}\right|^{-\nu_{ \pm}}
$$

onde $\Delta k^{\prime}=k^{\prime}-k_{\text {crit }}^{\prime}$. Para verificar se de fato ocorre uma transição de fase no sistema, ajusta-se a equação (3-10) em uma escala log-linear (de forma que o coeficiente angular deste ajuste corresponde a $\left.\xi^{-1}\right)$. Após obter o comprimento de correlação, faz-se um gráfico deste versus $\Delta k^{\prime}$, de onde nota-se que em vez de um comportamento divergente do comprimento de correlação, observa-se uma dependência linear $\left(R^{2}=0.9999998 \ldots\right)$ em termos da variação da ancoragem das bordas, o que acaba por gerar um valor finito para $\xi$ no ponto em que $k^{\prime}=k_{\text {crit }}^{\prime}$, mostrado na figura 3.6.

Com a hipótese de uma transição de fase descartada, o que realmente acontece com o sistema ao passar ao regime de temperaturas com o perfil suave para o perfil com cúspides? A resposta para esta pergunta é encontrada na análise da função de correlação entre as velocidades de pares vizinhos, $C_{v}$, que possui, ver figura 3.7 , valor zero no ponto $k^{\prime}=k_{\text {crit }}^{\prime}$, independentemente 


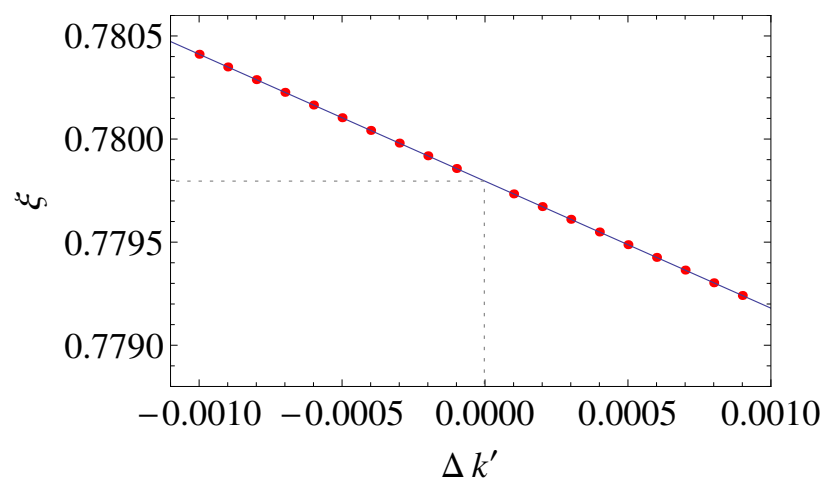

Figura 3.6: Comprimento de correlação como função de $\Delta k^{\prime}$. Os parâmetros utilizados são: $m=k_{1}=\gamma=1, k=\frac{1}{2}, T_{1}=1$ e $T_{N}=2$. Os pontos são obtidos analiticamente, e a reta traçada corresponde ao ajuste linear feito, cuja a inclinação é igual a $-0.61576 \pm 10^{-5}$ e o com o comprimento de correlação na origem igual a $\xi^{0}=0.779796 \pm 10^{-6}$.

da localização do par ao longo da cadeia.

Como pode se observar na figura 3.7, as correlações de velocidades são simétricas com respeito ao meio da cadeia, e uma análise geral sobre o comportamento destas curvas ajuda a desvendar a mecânica por trás da transição de regimes. Lembrando que as demais constantes de acoplamento foram escolhidas como $k=\frac{1}{2}$ e $k_{1}=1$, observa-se que para $k^{\prime}$ crescente, nas vizinhanças de $k_{\text {crit }_{1}}^{\prime}=1$, o valor de $C_{v_{1} v_{2}}$ passa de um valor negativo para um valor positivo e, simultaneamente, a temperatura da segunda partícula da cadeia, $\mathcal{T}_{2}$, também cresce monotonicamente (cf. Fig. 3.3).

A explicação para a mudança de comportamento das funções de correlação entre velocidades das partículas para diferentes valores de $k^{\prime}$ começa a ser construída ao notar-se o seguinte fato: a figura 3.7 mostra que o modo típico de vibração, considerando $k^{\prime}<1$, para elementos da cadeia mais próximos do reservatório frio é antissimétrico, enquanto partículas que se encontram mais perto do reservatório quente vibram no modo simétrico. O fator que define se as partículas encontram-se correlacionadas ou anti-correlacionadas é o sinal das correlações envolvendo os vizinhos da cadeia, o que permite interpretar com mais clareza a figura 3.7.

Utilizando os resultados das correlações entre velocidades em conjunto com a mudança de temperatura dos elementos da cadeia (cf. Fig. 3.3), é possível ainda inferir que os modos simétricos são mais energéticos do que os antissimétricos. Observa-se que quando aumenta-se ao valor de $k^{\prime}$ até chegar o valor no qual o sistema atravessa a primeira transição entre o regime suave e o que possui a cúspide, as temperaturas dos sítios da metade "mais fria" da cadeia acompanham tal crescimento. Por outro lado, a metade da cadeia mais 
Capítulo 3. O papel das correlações entre as velocidades nos perfis de temperatura do sistema

"quente" comporta-se de forma oposta, apresentando uma diminuição em suas temperaturas.

A mudança de comportamento das temperaturas de todas as partículas se mantém até o ponto em que $k^{\prime}=k_{\text {crit }}^{\prime}$, configuração na qual o volume do sistema atinge um platô de temperaturas e todos os vizinhos encontram-se descorrelacionados entre si. Como o sistema se encontra em um pseudo equilíbrio, é razoável encontrarmos que a correlação entre as velocidades seja nula ${ }^{1}$. Ao continuar aumentando o valor de $k^{\prime}$ após atingido o platô de temperaturas, nota-se que a metade da cadeia próxima ao reservatório frio passa a ter correlação de velocidades entre primeiros vizinhos positiva, enquanto a metade da cadeia mais próxima ao reservatório quente apresenta correlações negativas. Com isso a primeira transição de regimes de temperatura apresentada na cadeia se resume a uma inversão dos modos vibracionais das duas metades do sistema, onde verifica-se que a energia é transferida dos modos simétricos para os antissimétricos.

\footnotetext{
${ }^{1}$ Podemos chegar a essa conclusão olhando o caráter par da distruição de BoltzmannGibbs no sistema.
} 
Capítulo 3. O papel das correlações entre as velocidades nos perfis de temperatura do sistema
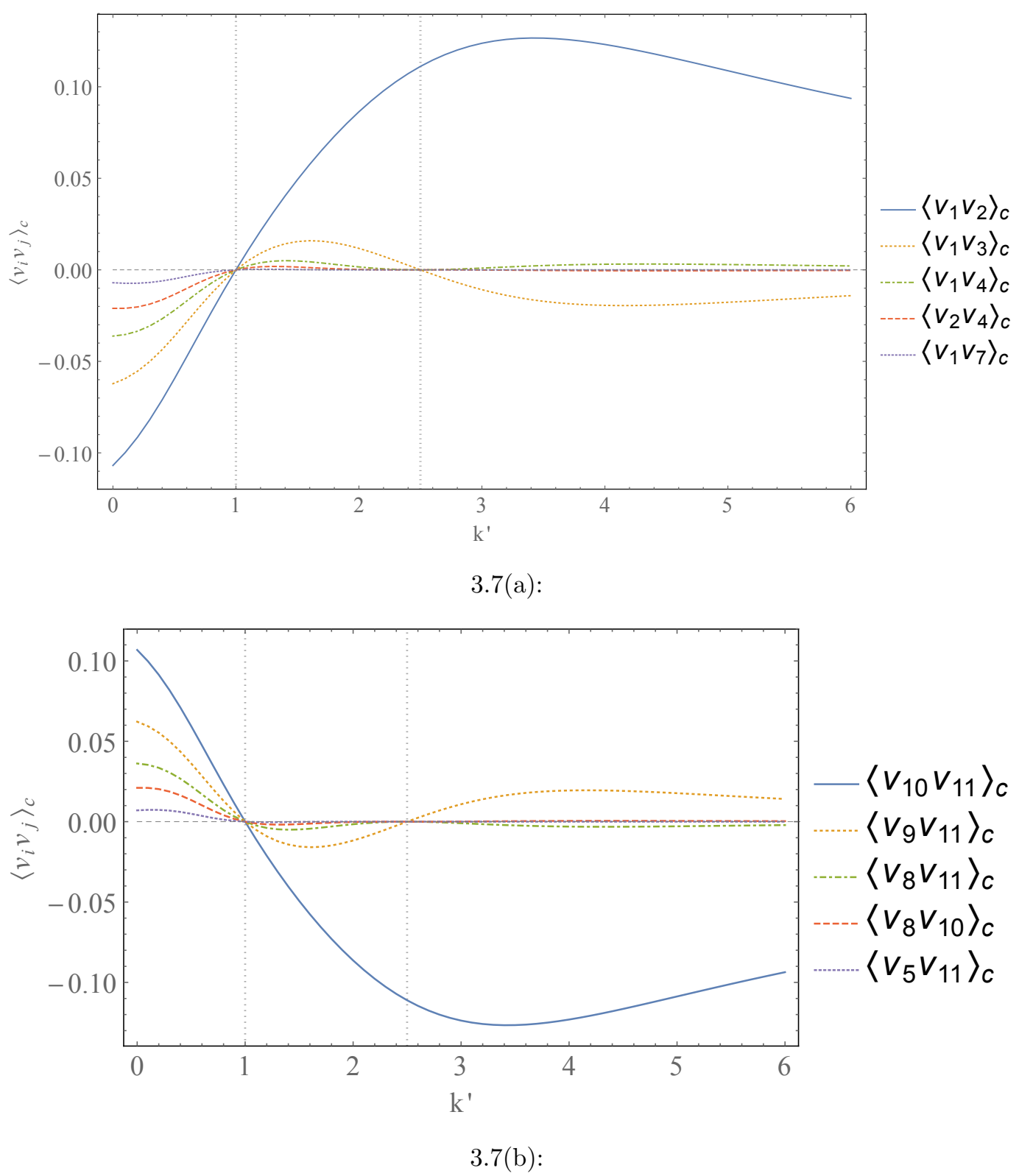

Figura 3.7: Correlação entre as velocidades de pares da cadeia, conforme indicado no lado direito de cada painel. Nos painéis superiores $k^{\prime}=1$ todas as correlações são nulas, e os elementos do volume encontram-se no platô de temperaturas. Quando $k^{\prime}=5 / 2$, o platô de temperaturas ressurge, bem como as correlações entre pares do volume se anulam, sendo as únicas exceções dadas por $C_{v_{1} v_{2}}$ e $C_{v_{N} v_{N-1}}$. Observe que as correlações são simétricas com relação ao meio da cadeia. Aqui utilizou-se uma cadeia com $\mathrm{N}=11$ elementos.

É importante frisar que toda esta discussão é válida em um sistema regido unicamentente por interações lineares. Quando termos de interação cúbica e/ou quártica encontram-se presentes na dinâmica do sistema, o transporte balístico de energia é modificado [31] , levando ao surgimento de gradientes de temperatura ao longo da cadeia. A discussão sobre algumas propriedades do 
fluxo de calor em cadeias não lineares possui uma vasta literatura associada a resultados obtidos via simulação numérica [25, 26, 66-68], enquanto o tratamento analítico para este tipo de modelo é abordado por poucos autores [69-71].

Para ilustrar um dos impactos das não linearidades sobre o sistema original, mostra-se na figura 3.8 o que ocorre com as correlações entre as velocidades quando introduz-se um termo cúbico de interação entre as partículas. Quando o termo $-k_{3} \sum_{j}\left(x_{i}-x_{j}\right)^{3}$ está presente nas equações de movimento, nota-se que as correlações não se anulam simultaneamente e a simetria que estas funções possuem com relação ao meio da cadeia é sutilmente quebrada. A técnica que conduz aos novos resultados das correlações é apresentada no capítulo 4 e apêndice E, porém pode se discutir de antemão qualitativamente os resultados obtidos.

O fato de todas as correlações não se tornarem nulas para qualquer par da cadeia e um dado $k^{\prime}$ em um modelo não-linear é simples de ser verificada. Uma das condições que definem a correlação zero é o pseudo equilíbrio do volume, por outro lado, como o sistema agora possui interações não-lineares, estas automaticamente inserem um gradiente de temperatura ao longo da cadeia, impossibilitando assim a formação de um platô de temperaturas para um determinado conjunto de parâmetros do problema.

Pode-se notar também que o termo cúbico favorece os modos antissimétricos da cadeia, visto que a maioria das correlações interpares $C_{v_{i} v_{j}}$ cruza o eixo $k^{\prime}$ após o valor $k_{\text {crit }}^{\prime}=1$. A discussão sobre os modos de vibração no caso não-linear para múltiplos valores de $k^{\prime}$ torna-se agora bem mais complicada, visto que a forma a qual eles interagem torna-se consideravelmente mais complexa devido às anarmonicidades do sistema. 
Capítulo 3. O papel das correlações entre as velocidades nos perfis de temperatura do sistema

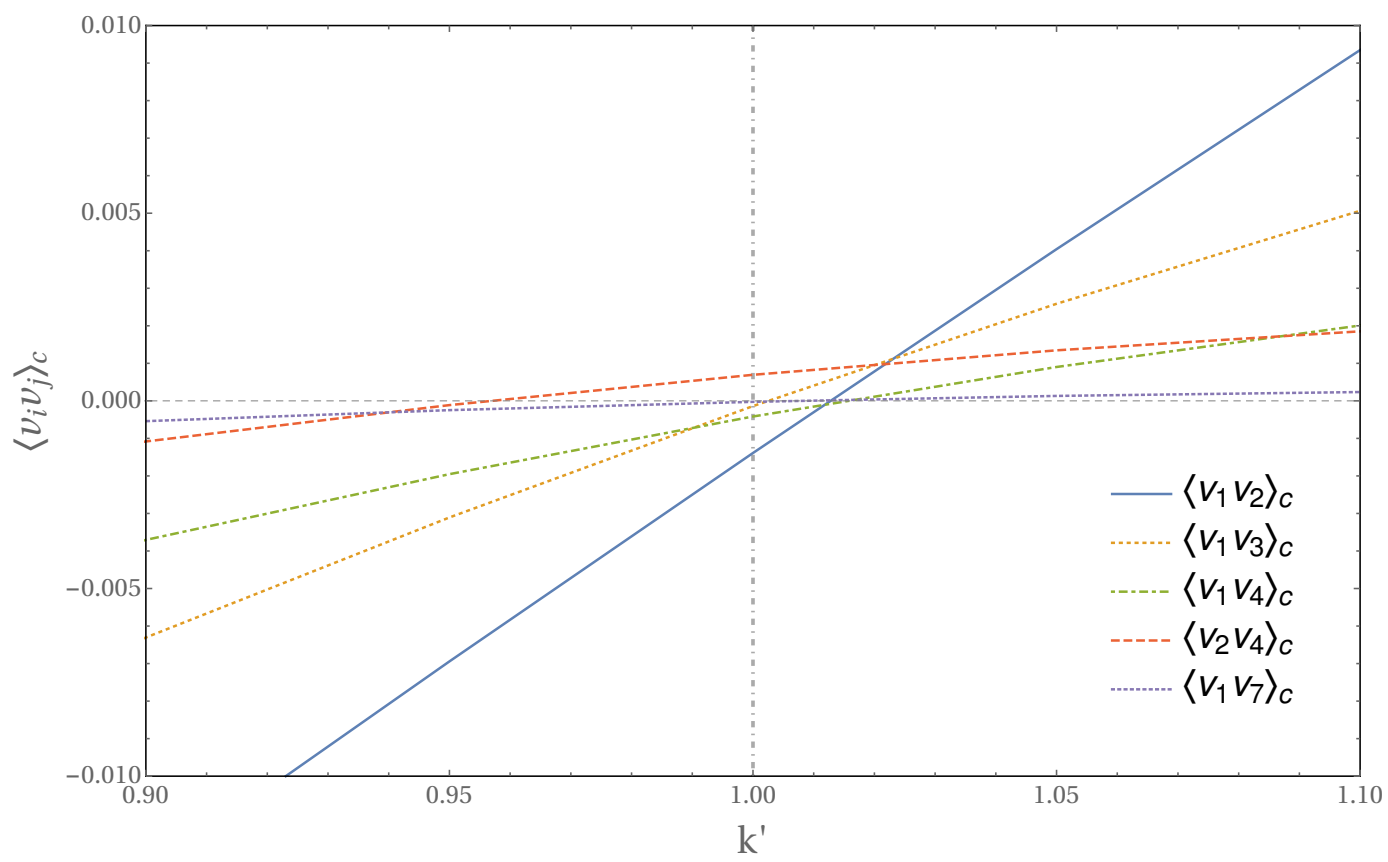

3.8(a):

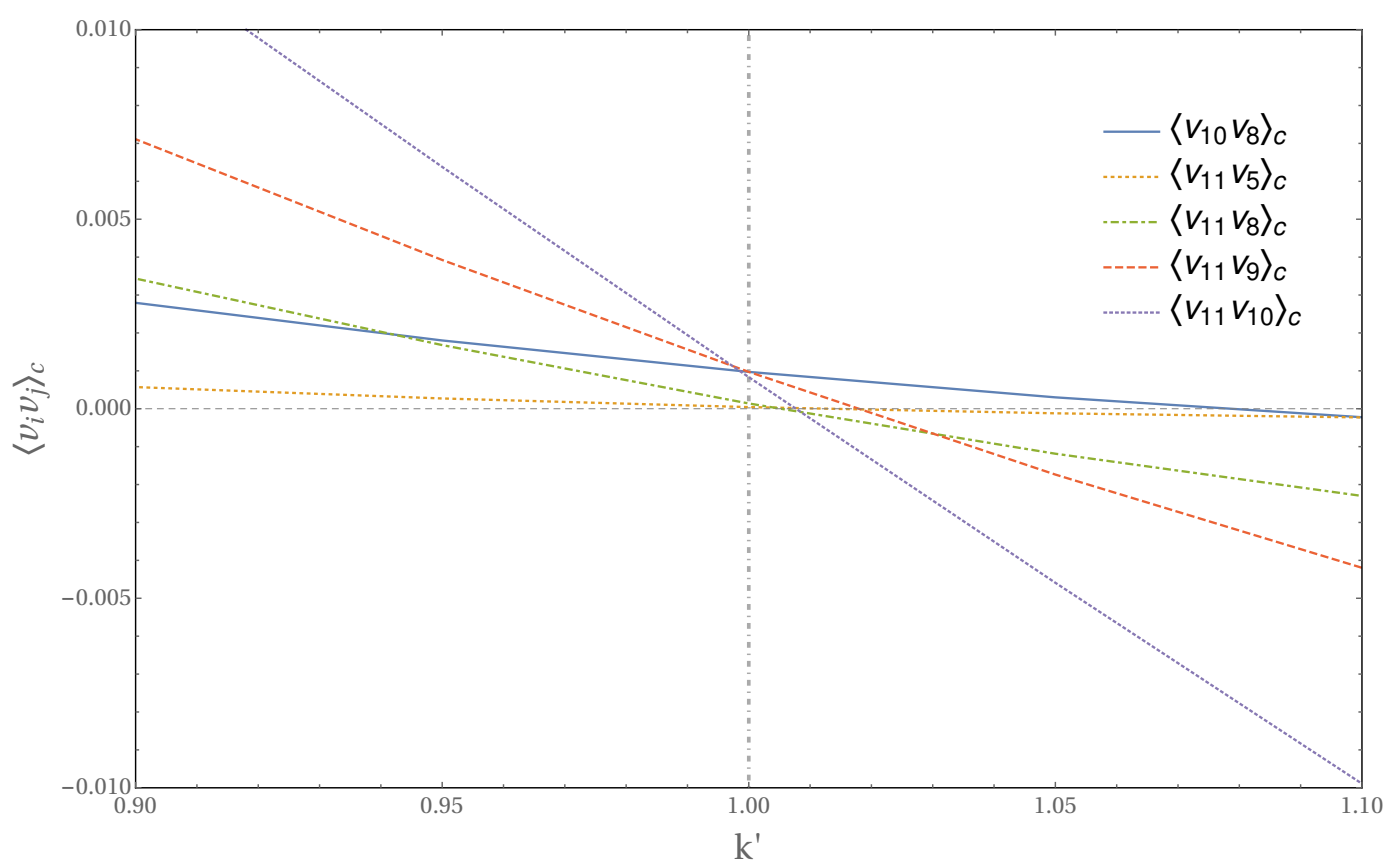

3.8(b): 
Capítulo 3. O papel das correlações entre as velocidades nos perfis de temperatura do sistema

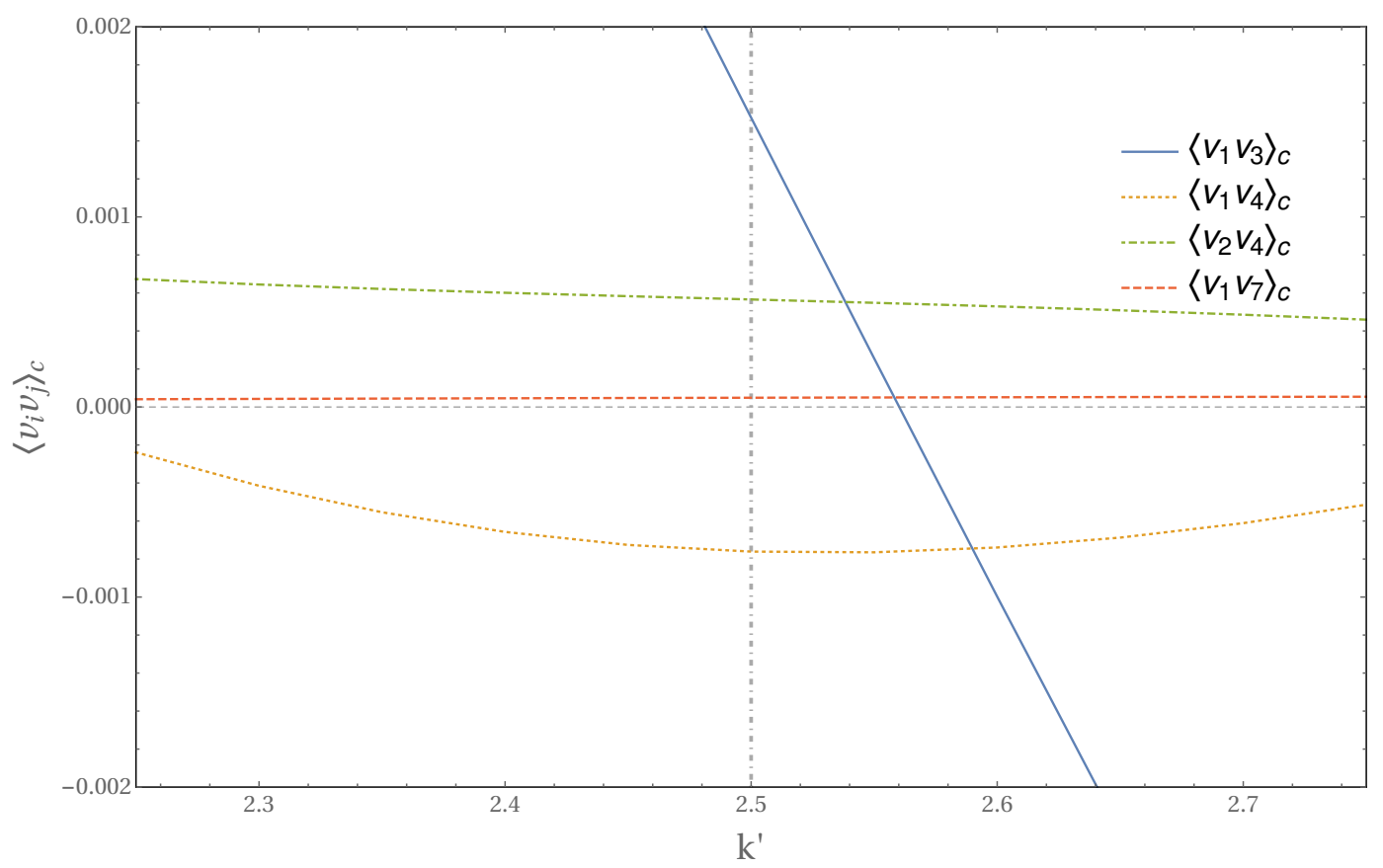

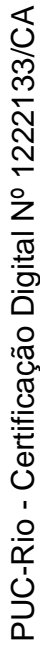

$3.8(\mathrm{c})$ :

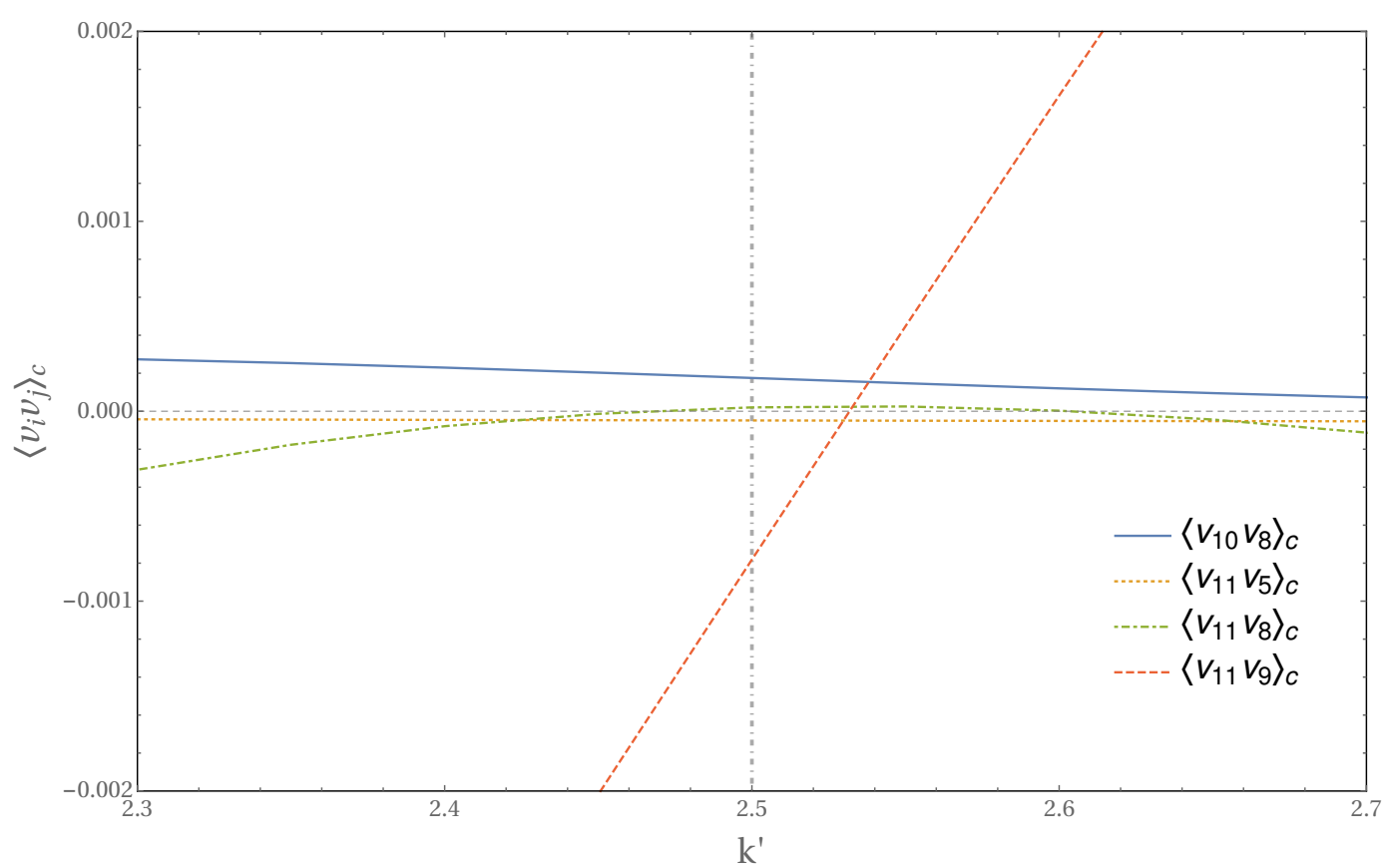

$3.8(\mathrm{~d}):$

Figura 3.8: Os painéis superiores (3.8(a) e 3.8(b)) mostram que as correlações atingem valor zero nas vizinhanças de $k_{\text {crit }}^{\prime}=1$. Nos painéis inferiores $(3.8(\mathrm{c}) \mathrm{e}$ $3.8(\mathrm{~d}))$ é possível notar que os resultados de correlações simultaneamente nulas em $k_{c r i t}^{\prime}=\frac{5}{2}$ não ocorrem na cadeia não-linear. Considerou-se uma cadeia com $\mathrm{N}=11$ elementos nesta análise. 


\section{O papel das anarmonicidades no fluxo de calor em cadeias alimentadas por reservatórios térmicos e atérmicos}

Como pôde ser verificado no último capítulo, modelos regidos apenas por interações harmônicas apresentam propriedades indesejadas e inconsistentes com o que se é observado em um sistema físico real. Para resolver tais falhas, é necessário considerar que as interações entre as partículas do sistema vão além da ordem harmônica, tornando então o sistema bem mais complexo de ser analisado.

Como alguns exemplos de modelos que consideram novos elementos na dinâmica do sistema, pode se destacar sistemas de rotores com interações de curto e longo alcance [72, 73], modelos que utilizam dispersão de massa [29, 60, 74], apresentam reservatórios auto-consistentes ligados aos elementos do volume [56, 75, 76], possuem interações não lineares entre os elementos da cadeia $[25,32,33,66,77]$ e ainda existem aqueles que misturam alguns destes novos elementos da dinâmica em um único modelo [59, 61, 71]. Dentre as possíveis mudanças que um sistema pode apresentar em suas propriedades de transporte quando sujeito a uma dinâmica não linear, pode se destacar uma que é comum em todos os modelos modificados citados: o espalhamento dos fônons, que é um fator primordial para satisfazer o caráter difusivo da lei de Fourier. A introdução de não-linearidades acaba tornando a análise destes modelos via simulação uma tarefa muito mais tratável e simples, sendo que as soluções analíticas para estes problemas desenvolvidas são obtidas poucas vezes [59, 61, 75].

A abordagem adotada ao longo desta seção é baseada em um modelo semelhante ao Fermi-Pasta-Ulam $\alpha-\beta$ (FPU- $\alpha-\beta)^{1}$, um problema sem soluções analíticas, que foi proposto no ano de 1955 por Enrico Fermi, John Pasta, Stan Ulam e Mary Tsingou, onde buscava-se verificar quão rápido um sistema não-linear de muitas partículas atinge o equilíbrio a partir de um determinado conjunto de condições iniciais. O estudo deste problema representou um grande avanço para as simulações numéricas[77], principalmente no campo da dinâmica molecular [25]. A hamiltoniana que define o modelo FPU pode ser escrita como:

\footnotetext{
${ }^{1}$ Embora o modelo seja creditado apenas a Fermi, Pasta e Ulam, a responsável pela escrita do código e pela realização das simulações é a cientista americana Mary Tsingou, que não teve o devido reconhecimento por este trabalho.
} 


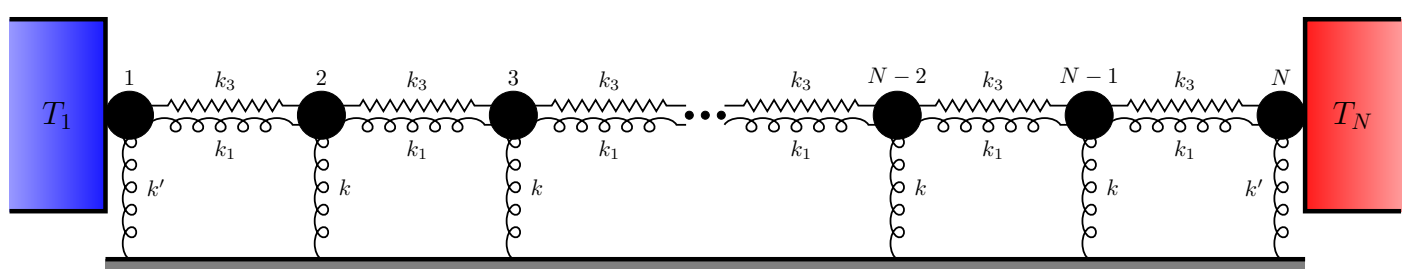

Figura 4.1: Cadeia contendo interações harmônicas e anarmônicas entre seus elementos.

$$
H=\sum_{i=1}^{N} \frac{p_{i}^{2}}{2 m}+\sum_{i=1}^{N-1} \frac{k\left(x_{i}-x_{i+1}\right)^{2}}{2}+\frac{\alpha\left(x_{i}-x_{i+1}\right)^{3}}{3}+\frac{\beta\left(x_{i}-x_{i+1}\right)^{4}}{4}
$$

Ao longo deste capítulo estuda-se o fluxo de calor um sistema onde somente as interações associadas aos potenciais quadráticos e quárticos estão presentes, o que representa uma variante do modelo FPU- $\beta$, i.e., não existem interações associadas a potenciais cúbicos. Um dos efeitos desta não linearidade foi mostrado brevemente ao final do capítulo 3 , onde mostrou-se que a presença desta acaba por tornar inatingível o platô de temperaturas e, consequentemente, o zero simultâneo entre as correlações de pares da cadeia. A dinâmica do sistema que fornece estes resultados é dada por

$$
\left\{\begin{array}{l}
m \frac{d^{2} x_{1}}{d t^{2}}=-\gamma \frac{d x_{1}}{d t}-k^{\prime} x_{1}-k_{1}\left(x_{1}-x_{2}\right)-k_{3}\left(x_{1}-x_{2}\right)^{3}+\eta_{1} \\
m \frac{d^{2} x_{i}}{d t^{2}}=-k x_{i}-k_{1}\left(2 x_{i}-x_{i+1}-x_{i-1}\right)-k_{3}\left(x_{i}-x_{i-1}\right)^{3}-k_{3}\left(x_{i}-x_{i+1}\right)^{3} \\
m \frac{d^{2} x_{N}}{d t^{2}}=-\gamma \frac{d x_{N}}{d t}-k^{\prime} x_{N}-k_{1}\left(x_{N}-x_{N-1}\right)-k_{3}\left(x_{N}-x_{N-1}\right)^{3}+\eta_{N}
\end{array}\right.
$$

Como não é possível resolver de forma direta este tipo de conjunto de equações, utiliza-se a técnica de teoria de perturbação com o intuito de obter a forma aproximada das soluções para as posições e velocidades das partículas, possibilitando assim o cálculo de grandezas tais como o fluxo de calor que atravessa a cadeia, temperaturas dos sítios, correlações entre pares, entre outras quantidades. A escolha para o parâmetro da expansão perturbativa resume-se a uma análise de duas das escalas típicas do problema, as energias associadas 
às interações linear e não linear, que podem ser escritas como:

$$
\begin{gathered}
U_{h}=\frac{k_{1} x^{2}}{2} \\
U_{n l}=\frac{k_{3} x^{4}}{4} .
\end{gathered}
$$

Intuitivamente deve se esperar que os resultados do regime não perturbado $\left(k_{3}=0\right)$ sejam recuperados quando o parâmetro perturbativo, daqui para frente designado por $\delta$, seja nulo. O parâmetro $\delta$ surge naturalmente na análise do problema quando impõe-se que, para a expansão perturbativa ser válida, é necessário que a escala de energia da interação harmônica seja muito menor do que a da interação cúbica, de forma que

$$
\frac{U_{n l}}{U_{h}} \ll 1 \rightarrow \frac{\frac{k_{3} x^{4}}{4}}{\frac{k_{1} x^{2}}{2}}=\frac{k_{3} x^{2}}{k_{1}} \sim \frac{k_{3} T}{k_{1}^{2}} \equiv \delta \ll 1,
$$

onde no penúltimo passo da equação (4-4), fez-se uso do teorema da equipartição de energia $\left(T \sim k_{1} x^{2}\right)$.

Após definir qual é o parâmetro de perturbação do sistema, é natural supor que o vetor que contêm a informação sobre a posição de todas as partículas pode ser escrito como

$$
\mathbf{x}_{i}=\mathbf{x}_{i}^{(0)}+\delta \mathbf{x}_{i}^{(1)}+\delta^{2} \mathbf{x}_{i}^{(2)}+\ldots
$$

Como o interesse é determinar a solução do problema em primeira ordem em $\delta$, todos os termos de ordem superior serão desconsiderados. Por se tratar de um desenvolvimento extenso, os seus detalhes encontram-se reportados no apêndice E. Ao longo do texto principal são mostrados apenas os resultados do método aplicado. Dentre as possíveis quantidades que podem ser estudadas utilizando as soluções das equações de movimento, neste capítulo enfatiza-se a análise do fluxo de calor através da cadeia quando esta encontra-se sob influência de reservatórios gaussianos e poissonianos.

Devido à presença da interação cúbica no sistema, o fluxo de calor não é mais dado por pela Eq. (2-6), mas deve ser calculado de acordo com[78]:

$$
\langle J\rangle_{i \rightarrow j}=\left\langle\left\{\left[-k_{1}\left(x_{i}-x_{j}\right)-k_{3}\left(x_{i}-x_{j}\right)^{3}\right] \frac{v_{i}+v_{j}}{2}\right\}\right\rangle,
$$


O desenvolvimento da Eq. (4-6) para os casos onde faz-se uso de (4-5) até primeira ordem é bastante trabalhoso, e, por este motivo, torna-se mais conveniente mostrar o cálculo do fluxo de calor para os ruídos gaussianos e poissonianos ao longo do apêndice E. Por hora, mostra-se apenas os resultados finais para a corrente de calor nos casos citados acima.

Os resultados serão ilustrados nas seções a seguir, que encontram-se divididas da seguinte forma: na primeira parte mostra-se qual é o efeito das não-linearidades em sistemas alimentados por reservatórios gaussianos; na segunda seção discute-se qual é a resposta da condutividade térmica quando o sistema tem energia injetada no seu interior por banhos poissonianos; na terceira e última parte, analisa-se propriedades da cadeia quando cada uma de suas extermidades encontra-se simultaneamente em contato com banhos térmicos de classes diferentes, o gaussiano e o de poissoniano.

\section{1}

\section{Reservatórios gaussianos (térmicos)}

Para determinar o fluxo no caso não-linear para o ruído gaussiano, continua-se a empregar a Eq. (2-18) para avaliar os cumulantes associados aos ruídos dos reservatórios, porém agora a presença destes termos não resultam apenas de médias quadráticas envolvendo os ruídos, mas também de fatores envolvendo médias quárticas dos mesmos, que são provenientes dos termos ligados ao parâmetro perturbativo $\delta$.

Sendo estes termos as únicas novidades que aparecem no cálculo, pode se trabalhar a Eq. (4-6) utilizando o teorema de Wick e manipulações algébricas, (cf. apêndices E e F), de maneira que o fluxo de calor pode ser escrito como:

$$
\begin{aligned}
& \langle J\rangle_{\mathcal{N} \mathcal{L} \mathcal{G}}= \\
& =\langle J\rangle_{\mathcal{L}}+6 \gamma^{2} k_{1} k_{3}\left\{\sum _ { j = 2 } ^ { N } \left[T_{1}^{2} \mathcal{I}_{4}^{[j]}\left(\mathcal{I}_{3}^{[j, j-1]}+\mathcal{I}_{7}^{[j, j-1]}\right)+T_{N}^{2} \mathcal{I}_{6}^{[j]}\left(\mathcal{I}_{5}^{[j, j-1]}+\mathcal{I}_{8}^{[j, j-1]}\right)+\right.\right. \\
& \left.+T_{1} T_{N}\left(\mathcal{I}_{3}^{[j, j-1]} \mathcal{I}_{6}^{[j]}+\mathcal{I}_{4}^{[j]} \mathcal{I}_{5}^{[j, j-1]}+\mathcal{I}_{6}^{[j]} \mathcal{I}_{7}^{[j, j-1]}+\mathcal{I}_{4}^{[j]} \mathcal{I}_{8}^{[j, j-1]}\right)\right]+ \\
& -\sum_{j=1}^{N-1}\left[T_{1}^{2} \mathcal{I}_{4}^{[j]}\left(\mathcal{I}_{3}^{[j, j]}+\mathcal{I}_{7}^{[j, j]}\right)+T_{1} T_{N}\left(\mathcal{I}_{3}^{[j, j]} \mathcal{I}_{6}^{[j]}+\mathcal{I}_{4}^{[j]} \mathcal{I}_{5}^{[j, j]}+\mathcal{I}_{6}^{[j]} \mathcal{I}_{7}^{[j, j]}+\mathcal{I}_{4}^{[j]} \mathcal{I}_{8}^{[j, j]}\right)+\right. \\
& \left.\left.+T_{N}^{2} \mathcal{I}_{6}^{[j]}\left(\mathcal{I}_{5}^{[j, j]}+\mathcal{I}_{8}^{[j, j]}\right)\right]\right\}+6 \gamma^{2} k_{3}\left[T_{1}^{2} \mathcal{I}_{4}^{[1]} \mathcal{I}_{1}+T_{1} T_{N}\left(\mathcal{I}_{4}^{[1]} \mathcal{I}_{2}+\mathcal{I}_{6}^{[1]} \mathcal{I}_{1}\right)+T_{N}^{2} \mathcal{I}_{6}^{[1]} \mathcal{I}_{2}\right] .
\end{aligned}
$$

Como pode se observar na Eq. (4-7) as integrais $\mathcal{I}_{n}^{[r]}, \mathcal{I}_{n}^{[j, l]}, \mathcal{I}_{1}$ e $\mathcal{I}_{2}$, cujas definições encontram-se no apêndice E, são provenientes da interação não linear 
que existe na cadeia visto que elas são proporcionais ao fator $k_{3}$. Para checar a validade do método da expansão perturbativa, pode se comparar os resultados analíticos com os obtidos via simulação numérica. De acordo com a figura 4.2 é possível notar que para uma cadeia com $N=10$ partículas, os dois métodos fornecem resultados que diferem por cerca de $0.2 \%$, mostrando que a eficiência do método já constatada em pequenos sistemas permanece válida [79]. Nas simulações utilizou-se o método trapeizóidal para determinar as soluções das equações diferenciais [80].

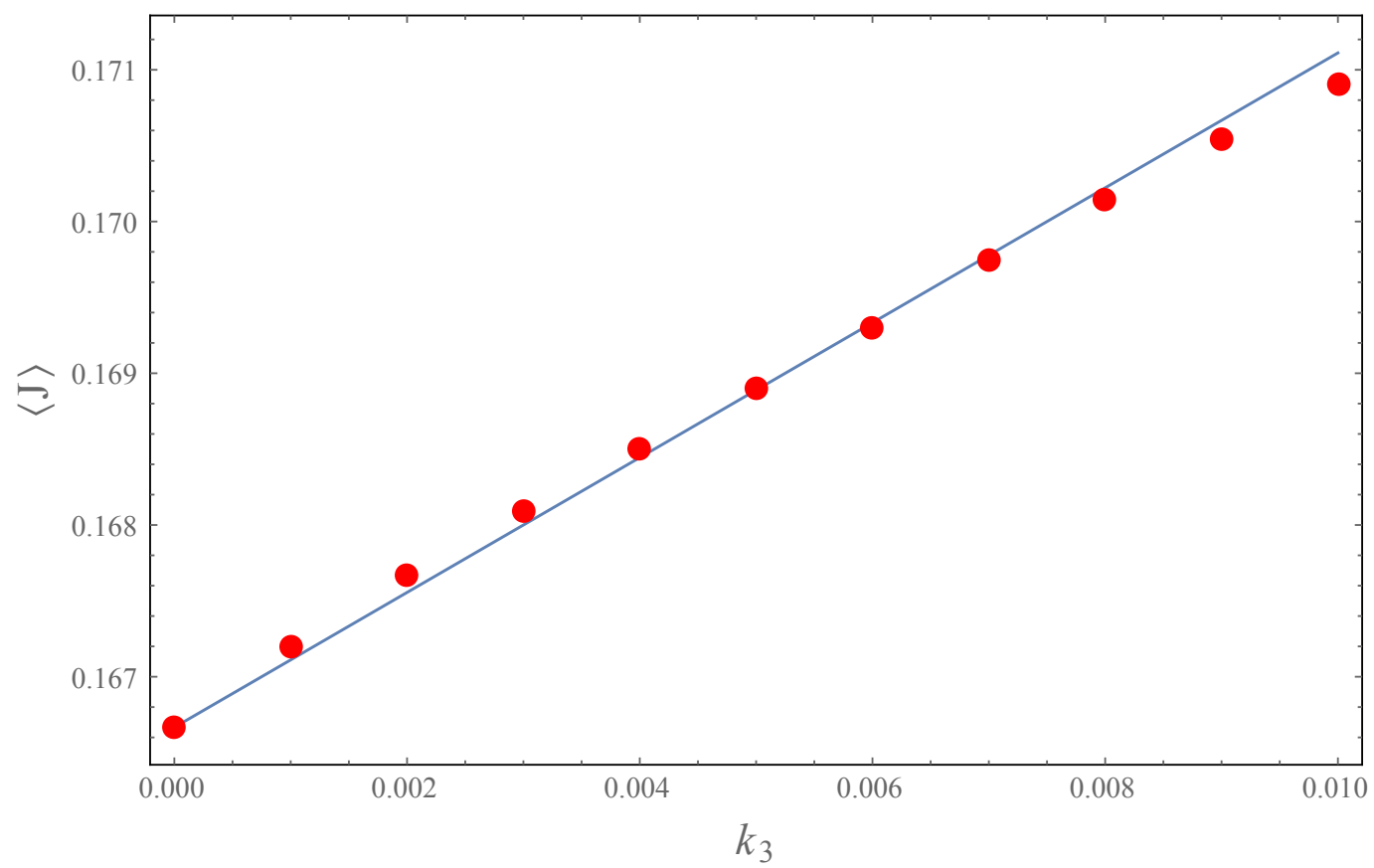

Figura 4.2: A corrente de calor para um sistema com $N=10$ partículas. A linha azul contnua representa o resultado analítico, enquanto os círculos vermelhos fornecem os dados da simulação Toma-se os parâmetros com os seguintes valores: $\gamma=m=k^{\prime}=k_{1}=1$ e $k=\frac{1}{2}$.

Como pode se observar na Fig. 4.2 o fluxo de calor vai aumentando linearmente com o parâmetro $k_{3}$, mostrando que as anarmonicidades aumentam a condutividade térmica do sistema. Este efeito pode ser entendido notando-se que como as partículas vizinhas encontram-se mais fortemente acopladas a transmissão de calor através das vibrações torna-se mais otimizada, o que aumenta a eficiência do transporte de energia ao longo da cadeia. 


\section{2}

\section{Reservatórios poissonianos (atérmicos)}

Recentemente, ruídos poissonianos vem atraindo grande atenção devido a sua aplicabilidade em um grande conjunto de fenômenos, tais como (i) problemas de estado sólido onde o ruído encontra-se correlacionado com a quantização da carga [81]; (ii) circuitos RLC cuja taxa de injeção de energia se assemelha a de bombas de calor [82]; (iii) motores biológicos onde o ruído mimetiza a hidrólise estocástica de não-equilíbrio da adenosina trisfosfato [83-85]; (iv) dinâmica molecular sujeita ao termostato de Andersen [86]; (v) gases granulares de baixa densidade empregados em sistemas de catracas [87-89]; (vi) a utilização de detectores à base de junções de Josephson, de modo a verificar cumulantes de ordem mais elevada em correntes flutuantes [90, 91];(vii) e estudo sobre flutuações de membranas das células vermelhas do sangue [92].

Motivado pelo vasto número de aplicações do ruído de Poisson, ao longo desta seção faz-se o estudo sobre fluxo de calor em cadeias alimentadas por este tipo de ruído, que pode ser definido como

$$
\eta(t)=\sum_{l} \Phi(t) \delta\left(t-t_{l}\right)
$$

Em um processo de Poisson é necessário definir as grandezas $\lambda(t)$, que define o número médio de eventos por unidade de tempo (no problema analisado estes eventos podem ser interpretados como "pancadas" que são dadas pelos reservatórios nas partículas que se encontram nas extremidades da cadeia) e $\Phi$, que corresponde a respectiva magnitudes de cada um dos eventos. No caso estudado considera- se que o processo seja homogêneo, ou seja, $\lambda(t)=\lambda$. Na Eq. (4-8) a grandeza $t_{l}$ representa os instantes de tempo em que ocorrem os eventos ("pancadas"). Embora seja possível usar um grande número de funções de distribuição para descrever $\Phi$, aqui restringe-se a análise da distribuição de probabilidades exponencial, que é dada por:

$$
P(\Phi)=\bar{\Phi}^{-1} \exp \left[-\frac{\Phi}{\bar{\Phi}}\right]
$$

com o momento de enésima ordem de $\Phi$ sendo calculado por $\overline{\Phi^{n}}=n ! \bar{\Phi}^{n}$. A última propriedade que define o processo de Poisson a ser utilizado diz 
respeito a sua correlação temporal

$$
\left\langle\eta\left(t_{1}\right) \ldots \eta\left(t_{n}\right)\right\rangle_{c} \equiv \lambda\left\langle\bar{\Phi}^{n}\right\rangle \prod_{i=1}^{n-1} \delta\left(t_{i+1}-t_{i}\right)
$$

No contexto de ruídos não-gaussianos a transformada de Fourier-Laplace continua sendo uma ferramenta de grande valia, de forma que ela continuará a ser utilizada nos cálculos subsequentes. Pode-se então escrever a equação (4-10) no espaço recíproco, onde esta assume a forma [93]:

$$
\left\langle\eta\left(\mathrm{i} q_{1}+\varepsilon\right) \ldots \eta\left(\mathrm{i} q_{n}+\varepsilon\right)\right\rangle_{c} \equiv \frac{\lambda\left\langle\bar{\Phi}^{n}\right\rangle}{\sum_{j=1}^{n}\left(\mathrm{i} q_{j}+\varepsilon\right)} \quad \forall_{n \in \mathbb{N}}
$$

A Eq. (4-11) é chave para entender a diferença entre os resultados fornecidos pelos casos poissoniano e gaussiano, visto que ela exprime o fato de o primeiro caso levar em consideração os seus infinitos cumulantes, enquanto o segundo é descrito apenas pelos seus dois primeiros e únicos cumulantes. A presença de novos cumulantes acaba por introduzir termos adicionais na Eq. (4-7), como por exemplo, os cumulantes quárticos, que surgem na teoria de perturbação em primeira ordem usada neste texto. Além de contribuírem na condução de calor do sistema [87], os novos cumulantes também modificam quantidades termoestatísticas associadas aos seus graus de liberdade [93].

Visando calcular o fluxo de calor, admite-se que a taxa de eventos, $\lambda$, de ambos os reservatórios seja idêntica, de forma que o fluxo de calor para um sistema que tem como fonte de energia reservatórios de Poisson e um número $N$ qualquer de partículas, é dado por:

$$
\begin{aligned}
& \langle J\rangle_{\mathcal{P}}=\langle J\rangle_{\mathcal{N} \mathcal{L} \mathcal{G}}+\left\{\sum_{j=2}^{N}\left[T_{1}^{2}\left(\mathcal{I}_{11}^{[j, j-1]}+\mathcal{I}_{13}^{[j, j-1]}\right)+T_{N}^{2}\left(\mathcal{I}_{12}^{[j, j-1]}+\mathcal{I}_{14}^{[j, j-1]}\right)\right]+\right. \\
& \left.-\sum_{j=1}^{N-1}\left[T_{1}^{2}\left(\mathcal{I}_{11}^{[j, j]}+\mathcal{I}_{13}^{[j, j]}\right)+T_{N}^{2}\left(\mathcal{I}_{12}^{[j, j]}+\mathcal{I}_{14}^{[j, j]}\right)\right]\right\} \frac{12 \gamma^{2} k_{1} k_{3}}{\lambda}+ \\
& +\frac{12 \gamma^{2} k_{3}}{\lambda}\left(T_{1}^{2} \mathcal{I}_{9}^{[1]}+T_{N}^{2} \mathcal{I}_{10}^{[1]}\right) .
\end{aligned}
$$

Nota-se da Eq. (4-12) que, mesmo avaliando-a no limite termodinâmico, não é possível eliminar a dependência no $\lambda$ associado ao reservatório de Poisson. A consistência da Eq. (4-12) pode ser verificada ao tomar-se o limite em que $\lambda \rightarrow \infty$, ou seja, quando a taxa de colisões se torna contínua, nota-se que 
o terceiro e quarto termos do lado direito vão a zero, recuperando assim o resultado obtido na Eq. (4-7).

\section{3}

\section{Reservatórios gaussianos e poissonianos}

As descrições do fluxo de calor e de outras grandezas termostatísticas em sistemas governados por reservatórios gaussianos e não-gaussianos, foram realizadas através de tratamento analítico em casos onde $N=1$ e $N=2$, donde pode observar-se alguns importantes resultados decorrentes da natureza dos reservatórios atérmicos $[79,87,93]$. Nestes trabalhos foi verificado que sistemas regidos por reservatórios deste tipo possuem características interessantes, tais como inaplicabilidade da lei zero da termodinâmica, dependência do fluxo de calor em outras propriedades dos banhos térmicos além da já existente com relação ao seu segundo cumulante e, por último, a introdução do conceito de temperaturas de ordem superior. A manifestação de todas estas características ocorre devido à combinação das não-linearidades presentes no sistema com a natureza dos reservatórios, permitindo assim que cumulantes de ordem superior a dois desempenhem um papel importante na dinâmica do sistema.

Um ponto intrigante a ser estudado em sistemas alimentados por reservatórios de naturezas distintas é a validade da $2^{\text {a }}$ lei da termodinâmica. Dentre as diferentes e equivalentes maneiras de enunciar tal lei, aqui coloca-se em xeque a formulação que foi proposta por Clausius [1, 3]: é possível uma fonte fria transferir calor para uma fonte quente sem que haja a realização de trabalho por um agente externo? A primeira investigação sobre esta questão foi feita por Kanazawa et al [87], onde mostrou-se que com ajustes adequados ao acoplamento cúbico, $k_{3}$, e as temperaturas canônicas dos reservatórios é possível observar a inversão do fluxo de calor no sistema. Este comportamento foi verificado inicialmente em um sistema composto por duas partículas e sob a hipótese de que as equações de movimento respeitassem o regime supercrítico ${ }^{2}$. Além de responder a questão sobre a violação da $2^{\text {a }}$ lei da termodinâmica, será mostrado ao longo desta seção que tais restrições não são necessárias para a ocorrência do fenômeno da inversão de calor, de maneira que o mesmo permanece válido para uma cadeia de tamanho arbitrário e que obedece às equações de movimento em sua forma mais geral possível.

Os passos matemáticos ao longo desta seção são omitidos, visto que o caso em que tem-se um reservatório gaussiano e um de Poisson representa uma situação particular da seção anterior. É simples se convencer desta afirmação

\footnotetext{
${ }^{2}$ Esta consideração supõe que o termo acompanhado razão $\frac{m}{\gamma}$ é desprezível nas eqs. de movimento do problema em relação aos demais.
} 
ao notar que o caso estudado pode ser obtido ao supor que a taxa de eventos de um dos reservatórios, $\lambda$, vai a infinito, o que transforma efetivamente um reservatório atérmico em um reservatório Gaussiano.

Para verificar o comportamento do fluxo de calor em sistema sob a ação de reservatórios de naturezas diferentes e para diferentes valores de $k_{3}$, toma-se o reservatório de não-equilíbrio como $R_{1}$, cuja temperatura canônica é $T_{1}=1.99$, enquanto o reservatório térmico, $R_{N}$, tem sua temperatura igual a $T_{N}=2.00$.

Em primeira análise a descrição do fluxo de calor mostrada na figura 4.3 parece fisicamente incorreta, pois aparentemente ocorrem violações da lei zero e da segunda lei da termodinâmica para determinados valores de $k_{3}$. Esta conclusão baseia-se na definição padrão de temperatura para um reservatório térmico, onde esta quantidade depende apenas do segundo cumulante do ruído. Na verdade não ocorre violação das leis da termodinâmica: a explicação do que ocorre advém da natureza do reservatório atérmico.

Quando considera-se sistemas alimentados por reservatórios atérmicos, deve-se atentar que a definição usual de temperatura não é mais aplicável. Isto deve-se ao fato de que banhos desta natureza possuem um número infinito de cumulantes. Consequentemente, no interior destes reservatórios tem-se produção contínua de entropia, o que impossibilita o equilíbrio térmico. Estas propriedades definem um reservatório de não-equilíbrio, cujos cumulantes de ordem superior acabam por desempenhar um importante papel na transferência de energia ao longo da cadeia.

Relembrando resultados obtidos no estudo de sistemas não lineares de uma partícula [79, 93], é possível verificar que os cumulantes de ordem superior atuam como fontes extras de energia, devido ao fato de que as contribuições para o fluxo de calor que são proporcionais a $\frac{k_{3}}{k_{1}^{2}} \lambda\left\langle\Phi^{n}\right\rangle=\frac{k_{3}}{k_{1}^{2}}\left\langle\eta^{n}\right\rangle_{c} \propto \mathcal{T}_{n}$, onde $\mathcal{T}_{n}$ é definido como temperatura de ordem $n$ sendo $\mathcal{T}_{2}$ coincidente com a temperatura canônica padrão $T$. O conceito de temperatura de ordem $n$ é construído através de uma analogia feita com o cumulante de segunda ordem da velocidade, bem como o da posição no caso linear, com a temperatura canônica de uma partícula. É possível mostrar que as temperaturas de ordem $n$ podem ser escritas como funções dos cumulantes das posições das partículas, que por sua vez podem ser escritas em termos dos cumulantes do ruído [93]. Vale notar que a definição destas temperaturas de ordem superior só faz sentido quando o reservatório que alimenta o sistema é de não-equilíbrio. 


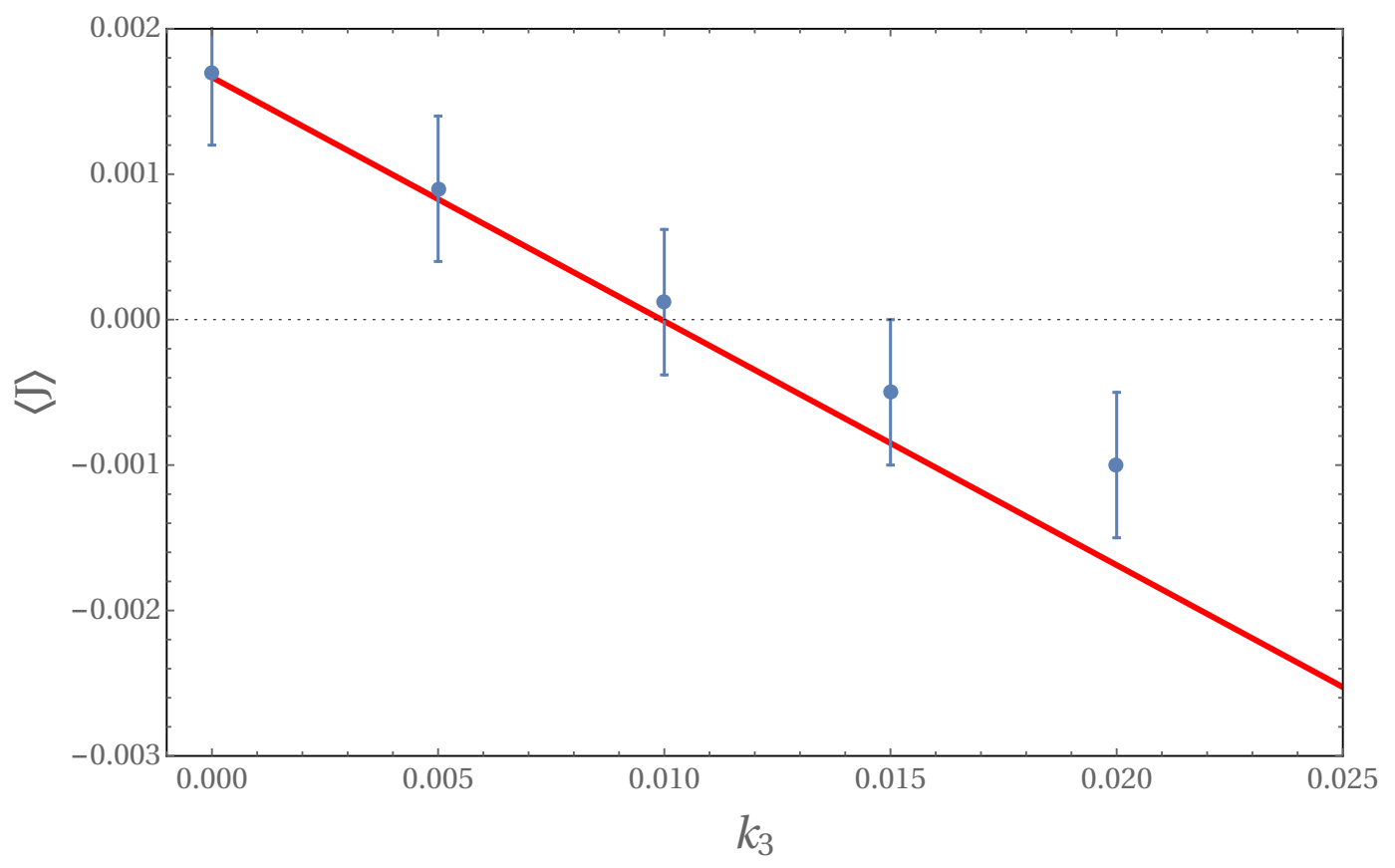

Figura 4.3: O fluxo de calor em uma cadeia com $N=10$ partículas. Faz-se a seguinte escolha para os parâmetros: $T_{N}=2, T_{1}=1.99, \lambda=4, \gamma=m=k^{\prime}=$ $k_{1}=1$ and $k=\frac{1}{2}$.

Além da motivação para um novo conceito de temperaturas, os cumulantes de ordem superior, em conjunto com as não-linearidades, também possuem influência sobre as funções resposta de um sistema, tal como o calor específico das partículas que também pode ter sua definição ampliada quando sistema em encontra-se alimentado por banhos atérmicos [93]. Para ilustrar um dos possíveis impactos destes cumulantes de ordem superior nas propriedades de transporte de uma cadeia mostra-se na figura 4.3 como a intensidade e o sentido do fluxo de calor estão relacionados com o grau de não-linearidade do sistema.

A física por trás do que acontece no sistema é desvendada quando responde-se à seguinte pergunta: qual a relação existente entre os cumulantes e as não linearidades? Pode-se responder esta pergunta ao se notar que os termos ligados a cumulantes de ordem superior encontram-se sempre acoplados com potências de $k_{3}$ [93], i. e., o efeito das não-linearidades e dos cumulantes de ordem superior influenciam de forma conjunta o fluxo de calor do sistema (ver apêndice E para mais detalhes). A afirmação anterior torna-se mais clara quando se verifica a contribuição dos cumulantes de quarta ordem na Eq. (4-12), que são representados pelos termos extras somados à corrente do caso gaussiano. 
Matematicamente, o efeito dos cumulantes de ordem superior no fluxo de calor em uma cadeia sob influência de um reservatório com ruído poissoniano simétrico e taxa de eventos $\lambda$ pode ser computado da seguinte forma:

$$
\begin{aligned}
\langle J\rangle_{\mathcal{P}} & =\sum_{n=0}^{\infty} k_{3}^{n}\left[\left\langle\Phi_{1}^{2 n+2}\right\rangle_{c}-\left\langle\Phi_{N}^{2 n+2}\right\rangle_{c}\right] \int C_{n}\left(\mathbf{q}, k_{1}, k, m, \gamma, \lambda\right) d \mathbf{q} \\
& =-\Lambda_{\operatorname{lin}} \Delta T+\sum_{n=1}^{\infty} \Lambda_{n}^{\prime}(\lambda) \Delta\left\langle\Phi^{2 n+2}\right\rangle_{c}
\end{aligned}
$$

onde $C_{n}\left(\mathbf{q}, k_{1}, k, m, \gamma, \lambda\right)$ são funções construídas a partir de combinações de elementos da matriz inversa da dinâmica do problema (ver apêndice E). Dado que momentos de enésima ordem estão relacionados com cumulantes de mesma ordem, e, como é possível escrevê-los últimos em função da temperatura de ordem $n$ [93], a Eq. (4-13) pode também ser expressa como

$$
\langle J\rangle_{\mathcal{P}}=-\Lambda_{\text {lin }} \Delta T+\sum_{n=1}^{\infty} \sum_{\mathrm{P}} \Lambda_{n}^{(\mathrm{P})}(\lambda) . \Delta \prod_{\{l\} \in \mathrm{P}} \mathcal{T}_{l},
$$

onde $\mathrm{P}$ corresponde ao número de partições possíveis de um bloco de tamanho $n,\{l\} \in \mathrm{P}$ fornece informação sobre os expoentes da partição e $\mathcal{T}_{l}$ a temperatura de ordem $n$.

As Eqs. (4-13) e (4-14) mostram que o efeito dos cumulantes depende da ordem da expansão perturbativa e do valor de $k_{3}$, o que limita a influência de termos gaussianos no problema, visto que estes se tornam menos relevantes para valores crescentes das não linearidades e cumulantes. É possível também ver que a inversão no fluxo de calor é um fenômeno que independe do tamanho da cadeia, uma vez que a Eq. (4-12) é desenvolvida para um sistema com um número qualquer de elementos.

A análise deste sistema também permite corroborar um resultado obtido em um caso particular do mostrado aqui [87]: a inversão do fluxo de calor depende apenas da natureza dos reservatórios envolvidos e do grau de não-linearidade presente nas interações da cadeia. Ainda com relação às Eqs. (4-13) e (4-14) pode se notar que, fisicamente, os cumulantes de uma distribuição ainda podem ser interpretados como fontes de energia internas aos reservatórios responsáveis pelo transporte de calor ao longo da cadeia, ou seja, esta conclusão independe do tamanho de sistema estudado. 


\section{5}

\section{Conclusões e perspectivas futuras}

No capítulo 2 propôs-se um modelo para descrever a transmissão de calor via condução, onde o sistema físico em questão é composto por partículas que interagem harmônicamente e dois reservatórios térmicos, de temperaturas $T_{1}$ e $T_{N}$, responsáveis pela injeção de energia na cadeia. O fato do sistema possuir apenas interações lineares permite que seja possível encontrar o comportamento de suas propriedades de transporte, como por exemplo o fluxo de calor. Supondo que a lei de Fourier seja válida para este sistema calcula-se a condutância térmica para cadeias de diferentes tamanhos, de onde pode se notar que o fluxo de calor tende a um valor de saturação enquanto aumenta-se o tamanho do sistema, o que implica em uma condutividade térmica infinita para o modelo ilustrado. Isto se deve ao fato de os fônons não sofrerem difusão, resultando assim em uma propagação sem espalhamento. A divergência da condutividade térmica indica que o sistema necessita possuir interações que vão além da harmônica, mostrando que um modelo demasiadamente simplório não é suficiente para descrever o fenômeno da condução de calor nos sólidos.

$\mathrm{Na}$ análise feita ao longo do capítulo 3 , que foi motivada pelo clássico modelo de condução de calor proposto por Rieder, Lebowitz e Lieb [27], ilustrou-se quantitativamente as relações existentes entre o tripleto composto pelas características mecânicas, o comportamento do fluxo de calor e a função de correlação entre as velocidades, com o intuito de descrever os possíveis perfis de temperatura presentes em cadeias harmônicas. Além do transporte de calor não difusivo, inerente a sistemas lineares, o modelo RLL tem outra característica marcante: o perfil de temperaturas se assemelha a um platô e apresenta uma cúspide (anti- cúspide) próxima ao reservatório frio (quente). Este perfil de temperatura é considerado contra-intuitivo, visto que a metade da cadeia que está mais próxima do reservatório frio encontra-se com uma temperatura superior que a da outra metade, que está próxima do reservatório quente. Foi mostrado então que no modelo RLL a razão para o surgimento das cúspide e anti-cúspide deve-se à falta de interação entre o volume do sistema e o substrato. Uma análise subsequente mostrou que a condição citada não é suficiente para gerar o perfil de temperatura do modelo RLL pois ao variar-se o valor da ancoragem de borda, $k^{\prime}$, constatou-se que é possível transicionar entre o regime monotônico para o regime cuspidal, e então voltar ao monotônico. Consequentemente, o aumento ou diminuição dos valores de ancoragem no 
espaço dos parâmetros é possível gerar um platô de temperaturas no volume do sistema, onde a temperatura de cada sítio é dada por $\mathcal{T}_{i}=\frac{T_{1}+T_{N}}{2}$ (para todo $2 \leqslant i \leqslant N-1$ ).

$\mathrm{Na}$ análise seguinte do capítulo 3, envolvendo as quantidades termoestatísticas da cadeia, descobriu-se uma notável propriedade do sistema quando $k^{\prime}=k_{\text {crit }}^{\prime}$, situação para o qual o volume se encontra no platô. Quando esta escolha de parâmetros é feita, a correlação entre as velocidades $v_{i} v_{j}$ para todos os pares da cadeia é zero. Efetivamente, o que ocorre é uma total descorrelação das partículas entre si e com os reservatórios. Ainda nesta configuração, observou-se que todos os cumulantes do fluxo de calor atingem seus extremos simultaneamente. Para um outro conjunto de parâmetros, com o seu respectivo $k^{\prime}=k_{\text {crit }}^{\prime}$, o platô de temperaturas surge novamente, bem como as correlações entre as velocidades vai a zero; particulamente, quando $k^{\prime} \rightarrow \infty$, os cumulantes ímpares tendem a zero, enquanto os pares atingem um valor de saturação, o que corresponde a um sistema isolado com temperatura $\frac{T_{1}+T_{N}}{2}$. Pode-se interpretar este resultado como se o sistema funcionasse como uma caixa de fônons, onde a função de correlação de velocidades é zero devido ao caráter par da distribuição de Boltzmann-Gibbs.

A coincidência de todos os cumulantes do fluxo de calor atingirem seus extremos para $k^{\prime}=k_{\text {crit }_{1}}^{\prime}$, em conjunto com uma mudança no perfil de temperatura do sistema, motivou a análise das funções de correlação entre as velocidades em regiões próximas à primeira transição de regimes, visando verificar uma possível transição de fase. Estudos iniciais envolvendo expoentes de Lyapunov em modelos de condutividade térmica [94], como por exemplo, os modelos de Toda [37] e ding-a-ling [28], indicaram comportamentos típicos de transição de fase associados a efeitos de tamanho finito. No modelo aqui apresentado, testou-se as relações cŕiticas em sistemas de diferentes tamanho, e o comportamento apresentado por eles foi idêntico em todos os casos. A evidência de que não existe transição de fase no sistema é dada pelo comportamento contínuo do comprimento de correlação relacionado ao quadrado das velocidades nas vizinhanças de $k_{\text {crit }}^{\prime}$, onde verficou-se um comportamento linear para este. Como não há transição de fase em $k^{\prime}=k_{\text {crit }_{1}}^{\prime}$, deve existir alguma explicação física para justificar a mudança no regime de temperaturas. A característica chave que permite explicar tal mudança se encontra na função de correlação entre as velocidades dos pares na cadeia, cujo sinal é trocado quando esta passa do regime RLL [27] para o perfil suave. A mudança no sinal da correlação entre as velocidades dá a informação sobre a redistribuição de modos da rede, de onde pode se notar que a metade da cadeia mais próxima do reservatório mais frio tem seus modos de vibração alterados 
de antissimétricos para simétricos, enquanto a outra metade se comporta de maneira oposta.

Ao final da seção 3.2 antecipou-se quais são os impactos das não-linearidades sobre os resultados obtidos ao longo do capítulo, de onde nota-se que esta modificação impede que a correlação das velocidades dos pares da cadeia se anule simultaneamente em um único ponto. Este efeito é causadopelo gradiente de temperaturas que surge no sistema devido à presença de interações não-lineares, impossibilitando assim a surgimento do platô, que é uma das consequências da total descorrelação entre as partículas da cadeia.

É importante frisar que, para um caso perturbativo em primeira ordem como o mostrado é possível recuperar a transição entre os regimes de temperaturas, bastando apenas que o parâmetro de acoplamento não linear do problema seja desprezível frente ao os acoplamentos lineares. No caso em que se resolve este problema através de simulação numérica e para valores quaisquer dos acoplamentos, fica claro que o platô não é mais atingível, visto que o programa leva em consideração todas ordens da expansão perturbativa.

Para que um modelo de condução de calor possua um caráter difusivo, é necessário que existam mecanismos de interação entre os fônons, como por exemplo, impurezas do material, efeitos de borda, espalhamento por elétrons e interações não lineares $[25,33,38,68]$. É conhecido que este último mecanismo citado é essencial para a formulação de quantidades associadas ao transporte de calor ao longo de um sólido [30,31], tais como a expansão térmica, o calor específico e a condutividade térmica. Buscando uma abordagem mais geral, no capítulo 4 inseriu-se tais interações não lineares entre os sítios da rede visando verificar a resposta do fluxo de calor que atravessa a cadeia. Através da teoria de perturbação desenvolveu-se uma expressão para o fluxo de calor válida para uma cadeia de tamanho genérico e sujeita a banhos de qualquer natureza.

Como uma extensão natural dos demais capítulos, o primeiro caso no qual foi feita a análise do fluxo de calor levou em consideração uma cadeia alimentada por reservatórios gaussianos, de onde verificou-se que a presença das novas interações produzem um aumento no fluxo de calor. Este efeito pode ser explicado devido à influência dos termos não-lineares ligados às médias quárticas que surgem na expressão para a energia injetada 4-7. Ao checar a expansão perturbativa apresentada em 4-5 em conjunto com a Eq. (E-1), pode se notar que caso a expansão fosse feita até segunda ordem em $k_{3}$, o fluxo de calor seria afetado também por médias de sexta ordem no ruído, ou seja, o fluxo de calor é proporcional às integrais ligadas a cumulantes de ordem par dos ruídos dos reservatórios.

Ao longo do quarto capítulo discutiu-se também o conceito de 
reservatórios de não-equilíbrio ou atérmicos, como exemplo o banho de Poisson. A definição de um reservatório atérmico pode ser feita em termos dos cumulantes associados ao ruído, visto que uma distribuição de probabilidades pode possuir apenas dois cumulantes, como no caso gaussiano, ou infinitos [95]. Os efeitos dos cumulantes de ordem superior a dois no fluxo de calor, presentes apenas em problemas onde existem interações não-lineares [79], afetam as propriedades termoestatísticas associadas ao sistema [93] e como foi ilustrado, podem produzir resultados que em uma primeira análise, aparentam ser fisicamente inconsistentes, como por exemplo a inversão do fluxo de calor. Mostrou-se que a ocorrência deste fenômeno não viola as leis fundamentais da termodinâmica, pois quando trabalha-se com reservatórios atérmicos o conceito usual de temperatura não se aplica, sendo então necessário entender o papel dos demais cumulantes da distribuição e também a interação física que estes possuem.

O real papel dos cumulantes de ordem superior pode ser entendido quando verificou-se a forma que estas quantidades acoplam-se com o parâmetro de não linearidade de rede, $k_{3}$. A Eq. (4-13) permite concluir que, embora o fluxo de calor dependa da parcela ligada aos cumulantes de ordem dois dos reservatórios, i.e. $\Delta T$, a contribuição dos cumulantes de ordem superior pode surpassar tal parcela dependendo da ordem da expansão perturbativa e do valor de $k_{3}$. Os cumulantes de ordem superior trazem consigo também o conceito de fontes de energia de ordem superior, este último fora introduzido em um trabalho para pequeno sistemas [93] e reapresentado ao longo do texto para cadeias de tamanho arbitrário.

Matematicamente, pode se entender o porquê da inversão do corrente de calor (cf. Eqs. 4-13 e 4-14), mas quando lembra-se do conceito da entropia associada ao sentido do fluxo de calor, pergunta-se onde é compensada a diminuição de entropia que ocorre quando o calor flui espontaneamente do reservatório mais "frio" para o mais "quente"? Embora não haja uma demonstração rigorosa, pode-se afirmar heuristicamente que o fator que explica a validade da segunda lei da termodinâmica neste caso encontra-se no interior do banho atérmico, que produz entropia continuamente, de forma que quando considera-se o sistema completo, a desordem deste é maior que zero.

Em suma o estudo em cadeias unidimensionais, lineares e não-lineares, representa um campo que ainda possui muitas oportunidades de pesquisa e também aplicações práticas, tais como experimentos que medem a condutividade térmica de nanofios $[13,14,41]$, nanotubos $[15,16,42]$ e até mesmo retificadores térmicos [96-98]. Uma perspectiva futura que permite uma abordagem mais ampla é verificar a extensão dos resultados obtidos em duas 
e três dimensões.

No caso de sistemas bidimensionais, de acordo com evidências númericas, o fluxo de calor em modelos anarmônicos apresenta uma condutividade térmica que diverge logaritmicamente com o tamanho da rede estudada [25, 38, 99], o que consequentemente faz com que a lei de Fourier não seja válida. Embora a princípio tais propriedades pareçam um ponto contra as redes bidimensionais, pode se utilizar estas para modelar sistemas físicos que possuem aplicação, tal como o grafeno, que é um material cuja condutividade térmica é muitíssimo elevada $[11,12]$.

Embora várias abordagens possam ser feitas, o enfoque a ser dado no estudo em sistemas de $2 \mathrm{D}$ simétricos seria em torno de o que acontece com a rede quando $k^{\prime}=k_{\text {crit }_{1}}^{\prime}$. É possível obter um platô de temperaturas nesta situação? Como se comporta a correlação de velocidades entre vizinhos? No caso de uma rede assimétrica, seria interessante descrever se é possível recuperar os resultados obtidos neste trabalho em $1 D$ ao fazer o número de elementos ao longo do eixo associado a transmissão de calor, por exemplo $\mathcal{N}_{x}$ ser muito maior que o número de elementos no eixo transverso, $\left(\mathcal{N}_{y}\right)$. É possível verificar que quando $\frac{\mathcal{N}_{y}}{\mathcal{N}_{x}} \rightarrow 0$ [100], o sistema deixa de apresentar uma condutividade térmica que diverge logaritmicamente, para uma divergência tipo lei de potências, o que é esperado no caso unidimensional. O ponto a ser investigado é verificar se existe alguma mudança na condição da razão $\frac{\mathcal{N}_{y}}{\mathcal{N}_{x}}$ quando o problema tiver seus parâmetros ajustados para a primeira transição de regimes, bem como checar qual as mudanças que ocorrem nas funções de correlação e nas propriedades de transporte do sistema.

O caso de transmissão de calor em modelos $3 D$ representa a situação mais interessante a ser investigada, por refletir todas as dimensões de um objeto físico real, porém mais complexo devido a modelagem do sistema a ser estudado e da matemática envolvida no processo. Embora a tarefa de estudar cadeias tridimensionais seja mais árdua, é possível obter resultados analíticos interessantes em modelos que envolvem apenas interações harmônicas entre primeiros vizinhos, tais como a função geratriz de cumulantes [46] associada e uma condutividade térmica que respeita a lei de Fourier, em um sistema onde se considera dispersão de massa ao longo da cadeia [63].

Em modelos não-lineares tridimensionais, verifica-se através de simulações numéricas que o transporte de calor obedece o previsto pela lei de Fourier, apresentando uma condutividade que depende do tamanho do sistema e o perfil linear de temperaturas. Além disso, é possível verificar a dependência da temperatura na condutividade térmica [66]. Vale frisar que este importante resultado é obtido considerando que as anarmonicidades em 
questão são da mesma ordem de grandeza que as interações lineares e um regime de baixas temperaturas, condições que propiciam uma investigação sob uma possível transição de fase entre o regime regular de calor e o anômalo. A existência de uma possível transição de fase entra nas perspectivas futuras de nosso trabalho e baseia-se no fato de que, se o parâmetro não-linear de interação for pequeno com relação à constante harmônica em um modelo $3 D$ [101], a condutividade térmica do sistema diverge, o que indica que deve haver uma relação entre $k_{3}$ e $k_{1}$ que delimite o transporte normal de calor e o transporte balístico.

Os problemas em aberto citados acima representam apenas uma pequena fração do que pode ser investigado na transmissão de calor via condução, o que torna esta área bastante atrativa no campo da física de não-equilíbrio. 


\section{A \\ A transformada de Fourier-Laplace e sua aplicação no cálculo do fluxo de calor}

Escolhe-se então a partícula 1 para aplicar a Eq.(2-6) sem nenhuma perda de generalidade. Antes de prosseguir introduz-se uma ferramenta matemática que fornece uma maneira alternativa para calcular-se a média temporal de uma função. Supondo que a distribuição $f(t)$ seja convergente é possível calcular a média temporal fazendo uso do teorema do valor final de forma que $[102,78]$ :

$$
\bar{f}=\lim _{z \rightarrow 0} z \tilde{f}(z)=\lim _{z \rightarrow 0} z \int_{0}^{+\infty} \exp [-z t] f(t) d t .
$$

Com o auxílio de (A-1) a Eq. (2-6) pode ser reescrita como

$$
\langle J\rangle=\lim _{z \rightarrow 0} z \int_{0}^{\infty} d t e^{-z t}\left(\left\langle\eta_{1}(t) v_{1}(t)-\gamma v_{1}^{2}(t)\right\rangle\right)
$$

Já a transformada de Fourier-Laplace de uma função g(t) é definida como:

$$
\widetilde{g}(i q+\epsilon) \equiv \int_{-\infty}^{0} g(t) e^{-(i q+\epsilon)} d t .
$$

A aplicação da Eq. (A-3) ficará mais simples de entender para o(a) leitor(a) ao longo desta seção. Analisando o termo de injeção de energia na Eq. (A-2) note que:

$$
\begin{gathered}
\left\langle\eta_{1}(t) v_{1}(t)\right\rangle=\int_{-\infty}^{\infty} \delta\left(t^{\prime}-t\right) d t^{\prime} \int_{-\infty}^{\infty} \delta\left(t^{\prime \prime}-t\right) d t^{\prime \prime}\left\langle\eta_{1}\left(t^{\prime}\right) v_{1}\left(t^{\prime \prime}\right)\right\rangle= \\
=\int_{0}^{\infty} \delta\left(t^{\prime}-t\right) d t^{\prime} \int_{0}^{\infty} \delta\left(t^{\prime \prime}-t\right) d t^{\prime \prime}\left\langle\eta_{1}\left(t^{\prime}\right) v_{1}\left(t^{\prime \prime}\right)\right\rangle,
\end{gathered}
$$

onde foi desconsiderado o intervalo de integração de menos infinito até zero visto que tanto a velocidade quanto o ruído são nulos para estes instantes de tempo. Observa-se que a Eq. (A-2) pode ser escrita de forma equivalente ao introduzir-se a delta de Dirac no integrando e aplicando a manipulação realizada na Eq. (A-4) tanto no termo de injeção de energia quanto no termo de dissipação: 
Apêndice A. A transformada de Fourier-Laplace e sua aplicação no cálculo do

fluxo de calor

$$
\begin{aligned}
\langle J\rangle= & \lim _{z \rightarrow 0} z \int_{0}^{\infty} d t e^{-z t} \int_{0}^{\infty} \delta\left(t^{\prime}-t\right) d t^{\prime} \int_{0}^{\infty} \delta\left(t^{\prime \prime}-t\right) d t^{\prime \prime}\left\langle\eta_{1}\left(t^{\prime}\right) v_{1}\left(t^{\prime \prime}\right)\right\rangle+ \\
& -\lim _{z \rightarrow 0} z \int_{0}^{\infty} d t e^{-z t} \int_{0}^{\infty} \delta\left(t^{\prime}-t\right) d t^{\prime} \int_{0}^{\infty} \delta\left(t^{\prime \prime}-t\right) d t^{\prime \prime}\left\langle v_{1}\left(t^{\prime}\right) v_{1}\left(t^{\prime \prime}\right)\right\rangle .
\end{aligned}
$$

Expressando as deltas de Dirac no integrando em termos de sua definição em termos da função exponencial, $\delta(t)=\int_{-\infty}^{\infty} \frac{d q}{2 \pi} e^{i q t}$, obtém-se

$$
\begin{array}{r}
\langle J\rangle=\lim _{z, \epsilon \rightarrow 0} z \int_{0}^{\infty} d t e^{-z t} \int_{0}^{\infty} \int_{-\infty}^{\infty} \frac{d q_{1}}{2 \pi} e^{\left(i q_{1}+\epsilon\right)\left(t^{\prime \prime}-t\right)} \frac{d q_{2}}{2 \pi} e^{\left(i q_{2}+\epsilon\right)\left(t^{\prime}-t\right)}\left\langle\eta\left(t^{\prime}\right) v_{1}\left(t^{\prime \prime}\right)\right\rangle d t^{\prime} d t^{\prime \prime}+ \\
\quad-z \int_{0}^{\infty} d t e^{-z t} \int_{0}^{\infty} \int_{-\infty}^{\infty} \frac{d q_{1}}{2 \pi} e^{\left(i q_{1}+\epsilon\right)\left(t^{\prime \prime}-t\right)} \frac{d q_{2}}{2 \pi} e^{\left(i q_{2}+\epsilon\right)\left(t^{\prime}-t\right)}\left\langle v_{1}\left(t^{\prime}\right) v_{1}\left(t^{\prime \prime}\right)\right\rangle d t^{\prime} d t^{\prime \prime} . \quad \text { (A-6) }
\end{array}
$$

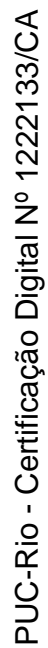

Na Eq. (A-6) suprimiu-se dois símbolos de integração em cada uma das parcelas com intuito de manter o texto mais esteticamente apresentável. Nota-se em (A-6) que as funções que se encontram entre $\langle\ldots\rangle$ ao serem integrados em suas respectivas variáveis de integração no limite de zero a infinito correspondem a transformada de Fourier-Laplace destas funções, o que permite reescrever (A-6) como:

$$
\langle J\rangle=\lim _{z, \epsilon \rightarrow 0} z \int_{0}^{\infty} d t e^{-z t} \int_{-\infty}^{\infty} \frac{d q_{1}}{2 \pi} \int_{-\infty}^{\infty} \frac{d q_{2}}{2 \pi} e^{\left(i q_{1}+i q_{2}+2 \epsilon\right) t}\left\langle\tilde{\eta}_{1}\left(i q_{2}+\epsilon\right) \tilde{v}_{1}\left(i q_{1}+\epsilon\right)\right\rangle-
$$

$$
-z \int_{0}^{\infty} d t e^{-z t} \int_{-\infty}^{\infty} \frac{d q_{1}}{2 \pi} \int_{-\infty}^{\infty} \frac{d q_{2}}{2 \pi} e^{\left(i q_{1}+i q_{2}+2 \epsilon\right) t}\left\langle\tilde{v}_{1}\left(i q_{2}+\epsilon\right) \tilde{v}_{1}\left(i q_{1}+\epsilon\right)\right\rangle
$$

Finalmente, integrando (A-7) no tempo encontra-se que o fluxo de calor é:

$$
\langle J\rangle=\lim _{z, \epsilon \rightarrow 0} \int_{-\infty}^{\infty} \frac{d q_{1}}{2 \pi} \int_{-\infty}^{\infty} \frac{d q_{2}}{2 \pi} \frac{z\left\langle\tilde{\eta}_{1}\left(i q_{2}+\epsilon\right) \tilde{v}_{1}\left(i q_{1}+\epsilon\right)-\tilde{v}_{1}\left(i q_{2}+\epsilon\right) \tilde{v}_{1}\left(i q_{1}+\epsilon\right)\right\rangle}{z-\left(i q_{1}+i q_{2}+2 \epsilon\right)} .
$$


B

Os operadores $\mathcal{D}(s)$ e $\mathcal{A}(s)$

A matriz que fornece a dinâmica da cadeia harmônica linear, $\mathcal{D}(s)$, no espaço de Laplace pode ser escrita como:

$$
\mathcal{D}(s)=
$$

$$
=\left(\begin{array}{cccccc}
m s^{2}+\gamma s+k_{1}+k^{\prime} & -k_{1} & \ldots & \cdots & \ldots & 0 \\
-k_{1} & m s^{2}+2 k_{1}+k & -k_{1} & \cdots & \ldots & 0 \\
0 & -k_{1} & \cdots & \cdots & \cdots & 0 \\
\vdots & \vdots & \ddots & -k_{1} & \ldots & 0 \\
\vdots & \vdots & \vdots & \ddots & \ldots & 0 \\
\vdots & \vdots & \vdots & \ddots & \ldots & 0 \\
0 & \cdots & 0 & -k_{1} & m s^{2}+2 k_{1}+k & -k 1 \\
0 & \cdots & 0 & 0 & -k_{1} & m s^{2}+\gamma s+k_{1}+k^{\prime}
\end{array}\right) \text {, }
$$

com sua inversa dada por,

$$
\begin{gathered}
\multicolumn{1}{c}{\mathcal{A}(s)=} \\
\left(\begin{array}{cccccc}
A_{11}(s) & A_{12}(s) & A_{13}(s) & \cdots & \cdots & A_{1 N}(s) \\
A_{21}(s) & A_{22}(s) & A_{32}(s) & \cdots & \cdots & A_{2 N}(s) \\
\vdots & \vdots & \ddots & \cdots & \cdots & \vdots \\
\vdots & \vdots & \vdots & \ddots & \cdots & \vdots \\
\vdots & \cdots & \cdots & \cdots & \ddots & \vdots \\
A_{N 1}(s) & \cdots & \cdots & \cdots & \cdots & A_{N N}(s)
\end{array}\right) .
\end{gathered}
$$

Os elementos $\mathcal{A}(s)_{i j}$ tem sua estrutura dada pela seguinte forma:

$$
\mathcal{A}(s)_{i j}=\frac{a_{0}+a_{1} s+a_{2} s^{2}+\ldots+a_{2 n-3} s^{2 n-3}+a_{2 n-2} s^{2 n-2}}{\operatorname{Det}[\mathcal{D}(s)]}
$$

Onde $n$ representa a dimensão do sistema, e $a_{k}$ são fatores constantes, diferentes para cada $\mathcal{A}(s)_{i j}$.

Com fins de ilustração, toma-se como exemplo o caso em que $N=4$, com os parâmetros $k^{\prime}=k=k_{1}=\gamma=m=1$. Tem se que $\mathcal{D}(s)$ e $\mathcal{A}(s)$ são escritos, 
respectivamente, como:

$$
\begin{gathered}
\mathcal{D}(s)=\left(\begin{array}{cccc}
s^{2}+s+2 & -1 & 0 & 0 \\
-1 & s^{2}+3 & -1 & 0 \\
0 & -1 & s^{2}+3 & -1 \\
0 & 0 & -1 & s^{2}+s+2
\end{array}\right) \\
\mathcal{A}(s)=\frac{1}{\operatorname{Det}[\mathcal{D}(s)]} \times\left(\begin{array}{cccc}
\mathcal{A}(s)_{11} & \mathcal{A}(s)_{12} & \mathcal{A}(s)_{13} & \mathcal{A}(s)_{14} \\
\mathcal{A}(s)_{21} & \mathcal{A}(s)_{22} & \mathcal{A}(s)_{23} & \mathcal{A}(s)_{24} \\
\mathcal{A}(s)_{31} & \mathcal{A}(s)_{32} & \mathcal{A}(s)_{33} & \mathcal{A}(s)_{34} \\
\mathcal{A}(s)_{41} & \mathcal{A}(s)_{42} & \mathcal{A}(s)_{43} & \mathcal{A}(s)_{44}
\end{array}\right)
\end{gathered}
$$

Onde $\operatorname{Det}[\mathcal{D}(s)]$ é:

$$
\operatorname{Det}[\mathcal{D}(s)]=s^{8}+2 s^{7}+11 s^{6}+16 s^{5}+40 s^{4}+38 s^{3}+54 s^{2}+26 s+21
$$

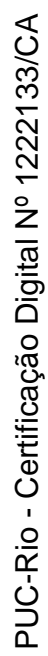

As entradas da matriz $\mathcal{A}(s)$, neste caso são escritas como

$$
\begin{aligned}
& \mathcal{A}(s)_{11}=\mathcal{A}(s)_{44}=s^{6}+s^{5}+8 s^{4}+6 s^{3}+19 s^{2}+8 s+13 \\
& \mathcal{A}(s)_{12}=\mathcal{A}(s)_{43}=s^{4}+s^{3}+5 s^{2}+3 s+5 \\
& \mathcal{A}(s)_{13}=\mathcal{A}(s)_{42}=2+s+s^{2} \\
& \mathcal{A}(s)_{14}=\mathcal{A}(s)_{41}=1 \\
& \mathcal{A}(s)_{21}=\mathcal{A}(s)_{34}=s^{4}+s^{3}+5 s^{2}+3 s+5 \\
& \mathcal{A}(s)_{22}=\mathcal{A}(s)_{33}=s^{6}+s^{5}+8 s^{4}+6 s^{3}+19 s^{2}+8 s+13 \\
& \mathcal{A}(s)_{23}=\mathcal{A}(s)_{32}=s^{4}+2 s^{3}+5 s^{2}+4 s+4 \\
& \mathcal{A}(s)_{24}=\mathcal{A}(s)_{31}=2+s+s^{2}
\end{aligned}
$$




\section{C \\ Fluxo de calor no limite termodinâmico}

Visando descrever o fluxo de calor em uma situação mais geral, mostra-se ao longo desta seção qual procedimento matemático deve ser seguido quando analisase uma cadeia no limite termodinâmico, i.e., $N \rightarrow \infty$. Ao invés de utilizar-se a transformada de Fourier-Laplace das equações de movimento do sistema, toma-se a transformada de Fourier por motivos que se tornarão claros ao longo da seção. A matriz B-1 pode ser escrita no espaço de Fourier como,

$$
\mathcal{D}(\omega)=
$$

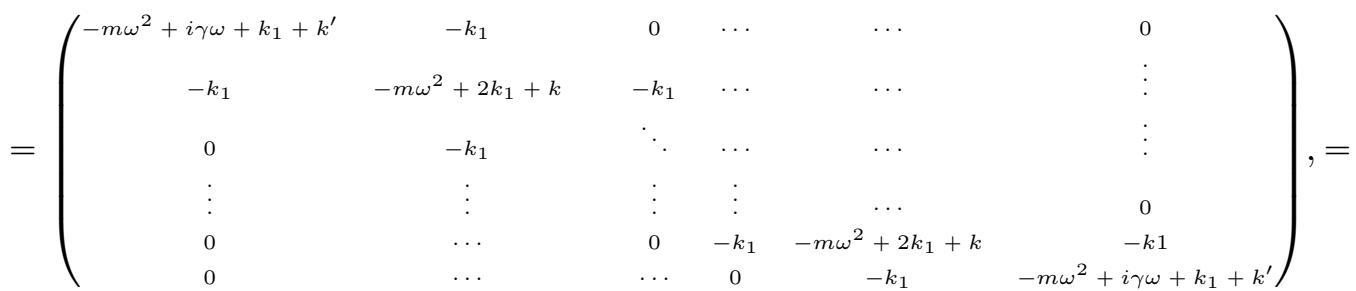

$$
=k_{1}\left(\begin{array}{cccccc}
\frac{-m \omega^{2}+i \gamma \omega+k^{\prime}}{k_{1}}+1 & -1 & 0 & \ldots & \ldots & 0 \\
-1 & \frac{-m \omega^{2}+k}{k_{1}}+2 & -1 & \ldots & \ldots & \vdots \\
0 & -1 & \ddots & \ldots & \ldots & \vdots \\
\vdots & \vdots & \vdots & \ddots & \ldots & 0 \\
0 & \cdots & 0 & -1 & \frac{-m \omega^{2}+k}{k_{1}}+2 & -1 \\
0 & \ldots & \ldots & 0 & -1 & \frac{-m \omega^{2}+i \gamma \omega+k^{\prime}}{k_{1}}+1
\end{array}\right)=
$$

$$
=k_{1}\left(\begin{array}{cccccc}
b-a & -1 & 0 & \cdots & \cdots & 0 \\
-1 & b & -1 & \cdots & \cdots & \vdots \\
0 & -1 & \ddots & \cdots & \cdots & \vdots \\
\vdots & \vdots & \vdots & \ddots & \cdots & 0 \\
0 & \cdots & 0 & -1 & b & -1 \\
0 & \cdots & \cdots & 0 & -1 & b-a
\end{array}\right) \equiv k_{1} \mathcal{Z}(\omega),
$$

onde $b=\frac{-m \omega^{2}+k}{k_{1}}+2, a=1+\frac{k-k^{\prime}-i \gamma \omega}{k_{1}}$. Como pode se inferir da equação (2-22), o fluxo de calor depende essencialmente do termo $\mathcal{A}(\omega)_{1 N}$, onde $\mathcal{A}(\omega)_{i j}$ é a matriz inversa de $\mathcal{D}(\omega)_{1 N}$ no espaço de Fourier. Este termo pode ser escrito como, [103]:

$$
\mathcal{A}(\omega)_{1 N}=\frac{1}{k_{1}}\left(\mathcal{Z}_{1 N}\right)^{-1}=\frac{1}{k_{1}} \frac{1}{\Delta_{N}},
$$


sendo $\Delta_{N}=\operatorname{Det}[\mathcal{Z}(\omega)]$. Com o intuito de encontrar uma forma fechada para $\Delta_{N}$, define-se o determinante $\Delta_{l, m}$ de uma submatriz de $\mathcal{Z}$, começando com a l-ésima linha e terminando na $m$-ésima coluna [45], como pode ser visto abaixo.

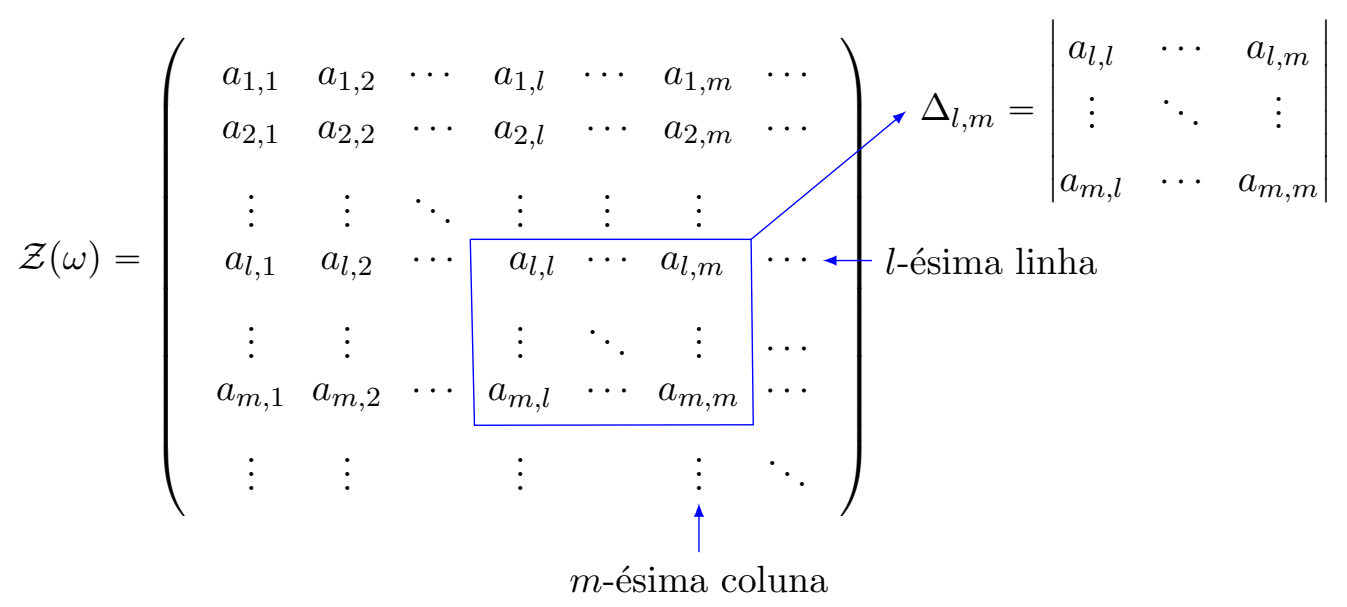

Seja agora $\mathcal{Z}_{0}$ uma submatriz de $\mathcal{Z}$ com dimensão $N-1 \times N-1$, construída removendo as primeiras e enésimas linhas/colunas da matriz $\mathcal{Z}$, sendo então esta submatriz formada por elementos na diagonal principal desta matriz iguais a $b$ e os elementos fora da diagonal iguais a -1 . De forma análoga ao que foi feito com $\Delta_{l, m}$ e $\mathcal{Z}$, define-se agora $\mathcal{R}_{l, m}$ como sendo o o determinante de uma submatriz de $\mathcal{Z}_{0}$ começando com a $l$-ésima linha e terminando na $m$-ésima coluna. Para encontrar uma expressão mais condensada para $\Delta_{N}$, utiliza-se as definições de $\Delta_{l, m}$ e $\mathcal{R}_{l, m}$, para construir-se as seguintes relações de recursão:

$$
\left\{\begin{array}{l}
\Delta_{1, N}=(b-a) \Delta_{2, N}-\Delta_{3, N} \\
\Delta_{2, N}=(b-a) \mathcal{R}_{2, N-1}-\mathcal{R}_{2, N-2} \\
\Delta_{3, N}=(b-a) \mathcal{R}_{3, N-1}-\mathcal{R}_{3, N-2} \\
\mathcal{R}_{1, N}=b \mathcal{R}_{2, N}-\mathcal{R}_{3, N} \\
\mathcal{R}_{2, N}=b \mathcal{R}_{2, N-1}-\mathcal{R}_{2, N-2}
\end{array},\right.
$$

onde $\Delta_{1, N} \equiv \Delta_{N}$. Assim após um pouco de manipulação algébrica, tem-se que o determinante da matriz inversa da dinâmica do problema é escrito como:

$$
\Delta_{N}=\mathcal{R}_{1, N}-a\left(\mathcal{R}_{2, N}+\mathcal{R}_{1, N-1}\right)+a^{2} \mathcal{R}_{2, N-1} .
$$

Observe que os determinantes da forma $\mathcal{R}_{i, j}$ presentes na Eq. (C-5) podem ser calculados analiticamente, visto que os elementos da diagonal principal destas matrizes são idênticos e constantes, bem como os termos que se encontram fora da diagonal principal. Para calcular o determinante da matriz $\mathcal{R}_{1, N}$ pode se utilizar 
a quarta Eq. mostrada em (C-4), de onde nota-se que [103, 104]:

$$
\mathcal{R}_{1, N}=C \lambda^{N}
$$

onde $C$ é uma constante. Substituindo em na quarta Eq. de (C-4) encontra-se a equação característica a seguir,

$$
\lambda^{2}-b \lambda+1=0
$$

com raízes:

$$
\lambda_{ \pm}=\frac{b \pm \sqrt{b^{2}-4}}{2}
$$

Por simplicidade faz-se

$$
\frac{-m \omega^{2}+k}{k_{1}}+2=b=2 \cos \theta
$$

assegurando que as raízes da equação sejam escritas de uma maneira mais simples, como $\lambda_{ \pm}=e^{ \pm \theta}$. Logo a solução mais geral para o determinante de $\mathcal{R}_{1, N}$ é escrita como:

$$
\mathcal{R}_{1, N}=C_{1} \lambda_{+}^{N}+C_{2} \lambda_{-}^{N}=C_{1} e^{N i \theta}+C_{2} e^{-N i \theta}
$$

É simples ver que $\mathcal{R}_{1,1}=b$ e $\mathcal{R}_{1,2}=b^{2}-1$, o que permite ajustar as condições de contorno associadas à $C_{1}$ e $C_{2}$, que agora podem ser representados da seguinte forma:

$$
C_{1}=\frac{e^{i \theta}}{e^{i \theta}-e^{-i \theta}}, C_{2}=-\frac{e^{-i \theta}}{e^{i \theta}-e^{-i \theta}}
$$

Substituindo (C-11) em (C-10), tem-se:

$$
\mathcal{R}_{1, N}=\frac{\sin [(N+1) \theta]}{\sin \theta} .
$$

Com a ajuda da Eq. (C-12) é possível escrever (C-5) em um formato mais elegante, dado por:

$$
\Delta_{N}=\frac{r(\theta) \cos N \theta+u(\theta) \sin N \theta}{\sin \theta}
$$

onde

$$
\begin{gathered}
r(\theta)=\left(1-a^{2}\right) \sin \theta, \\
u(\theta)=\left(1+a^{2}\right) \cos \theta-2 a .
\end{gathered}
$$

Agora a Eq. (2-22) no espaço de Fourier assume a seguinte forma:

$$
\langle J\rangle_{\mathcal{L}}=\frac{\gamma^{2} \Delta T}{k_{1}^{2} \pi} \int_{-\infty}^{\infty} \frac{\omega^{2} d \omega}{\left|\Delta_{N}\right|^{2}} .
$$

Observe que a Eq. (C-9) não se resume apenas a uma simples mudança de variável, ela também restringe os valores possíveis que as frequências do sistema podem assumir dentro do conjunto dos reais, isto é, para que $\omega$ seja real, a desigualdade $\frac{k}{m}<\omega^{2}<\frac{k+4 k_{1}}{m}$ deve ser válida, implicando assim que $\theta \in(0, \pi)$. A restrição para autofrequências reais torna-se mais clara e pertinente quando avalia-se na equação 
(C-15) no limite termodinâmico, onde verifica-se que para qualquer $\theta$ imaginário, o integrando da equação citada decai exponencialmente. Fazendo uma mudança de variável na equação (C-15), tem-se:

$$
\langle J\rangle_{\mathcal{L}}=\frac{\gamma^{2} \Delta T}{k_{1}^{2} \pi} \int_{-\infty}^{\infty} \frac{\omega^{2} d \omega}{\left|\Delta_{N}\right|^{2}}=\frac{\gamma^{2} \Delta T}{k_{1}^{2} \pi} \int_{0}^{\pi}\left|\frac{d \omega}{d \theta}\right| \frac{\omega^{2}}{\left|\Delta_{N}\right|^{2}} d \theta .
$$

Como o interesse é determinar uma expressão para a energia injetada quando $N \rightarrow \infty$, faz-se o uso da identidade a seguir [45],

$$
\lim _{N \rightarrow \infty} \int_{0}^{\pi} \frac{h(\theta)}{1+g(\theta) \sin N \theta} d \theta=\int_{0}^{\pi} \frac{h(\theta)}{\left[1-g^{2}(\theta)\right]^{\frac{1}{2}}} d \theta
$$

onde $h(\theta)$ e $g(\theta)$ são funções suaves. No caso estudado pode se concluir que as funções envolvidas satisfazem estas condições, visto que tanto o numerador quanto o denominador são compostos por combinações de funções seno e cosseno. O lado direito da Eq. (C-17), pode ser verificado notando que o seu integrando pode ser escrito como uma série geométrica, dada por:

$$
h(\theta)\left[1-g(\theta) \sin N \theta+g^{2}(\theta) \sin ^{2} N \theta+\ldots\right] d \theta .
$$

Agora nota-se que as potências da função seno que surgem na série são dadas por,

$$
\left\{\begin{array}{l}
n \text { ímpar } \rightarrow \sin ^{n} N \theta=\frac{2}{2^{n}} \sum_{l=0}^{\frac{n-1}{2}}(-1)^{\frac{n-1}{2}-l}\left(\begin{array}{l}
n \\
l
\end{array}\right) \sin [(n-2 l) N \theta] \\
n \text { par } \rightarrow \sin ^{n} N \theta=\frac{1}{2^{n}}\left(\begin{array}{c}
n \\
\frac{n}{2}
\end{array}\right)+\frac{2}{2^{n}} \sum_{l=0}^{\frac{n-1}{2}}(-1)^{\frac{n-1}{2}-l}\left(\begin{array}{l}
n \\
l
\end{array}\right) \cos [(n-2 l) N \theta]
\end{array}\right.
$$

Verifica-se em (C-19) que as contribuições que contêm potências ímpares da função seno são nulas, devido ao intervalo de integração, o que restringe o cálculo apenas aos termos ligados às potências pares. Assim fazendo uso de (C-19) e relações de arco dobro, tem-se que:

$$
h(\theta)\left[1+\frac{g^{2}(\theta)}{2}+\frac{3 g^{4}(\theta)}{8}+\ldots\right]=\frac{h(\theta)}{\left[1-g^{2}(\theta)\right]^{\frac{1}{2}}},
$$

concordando assim com a Eq. (C-17). Fazendo algumas manipulações e simplificações algébricas, pode se escrever:

$$
\left|\Delta_{N}\right|^{2}=\left(|r|^{2}+|u|^{2}\right) \frac{1+d \sin (2 N \theta+\phi)}{2 \sin ^{2} \theta},
$$

onde $d \cos \phi=\frac{r^{*} u+r u^{*}}{|r|^{2}+|u|^{2}}$ e $d \sin \phi=\frac{|r|^{2}-|u|^{2}}{|r|^{2}+|u|^{2}}$. Observe que C-21 possui a mesma estrutura do lado esquerdo da equação C-17, o que permite aplicar esta equação para desenvolver a integral em questão. Com isso, pode se escrever o fluxo de 
calor como:

$$
\langle J\rangle_{\mathcal{L}}=\frac{\gamma k_{1}^{2} \Delta T}{\pi m} \int_{0}^{\pi} \frac{\sin ^{2} \theta}{\Pi-\Theta \cos \theta} d \theta
$$

Integrando a Eq. (C-22), obtém-se o resultado apresentado no capítulo 2 através da equação (3-7). 


\section{D}

\section{Correlações de velocidades no limite termodinâmico}

Para encontrar a correlação entre as velocidades de duas partículas quaisquer pode se fazer uma analogia com a Eq. (3-3), de onde pode se concluir que a quantidade de interesse é dada por:

$$
\left\langle v_{i} v_{l}\right\rangle=\frac{\gamma}{\pi} \sum_{j=1, N} T_{j} \int_{-\infty}^{+\infty}(\mathrm{i} q+\varepsilon)(-\mathrm{i} q-\varepsilon) \widetilde{\mathcal{A}}_{i j}(\mathrm{i} q+\varepsilon) \widetilde{\mathcal{A}}_{l j}(-\mathrm{i} q-\varepsilon) d q .
$$

Em vez de desenvolver a Eq. (D-1), reescreve-se esta equação no espaço de Fourier onde a determinação desta expressão no limite termodinâmico é realizada de maneira mais simples. É simples notar que a expressão que define a correlação entre velocidades de duas partículas no espaço de Fourier é escrita como:

$$
\left\langle v_{i} v_{l}\right\rangle=\frac{\gamma}{\pi} \sum_{j=1, N} T_{j} \int_{-\infty}^{+\infty} \omega^{2} \widetilde{\mathcal{A}}_{i j}(\omega) \widetilde{\mathcal{A}}_{l j}(-\omega) d \omega .
$$

Recorrendo a notação determinada no apêndice C é simples verificar que [36]:

$$
\begin{aligned}
\widetilde{\mathcal{A}}_{i 1}(\omega) & =\frac{\Delta_{i+1, N}(\omega)}{k_{1} \Delta_{N}} \\
\widetilde{\mathcal{A}}_{i N}(\omega) & =\frac{\Delta_{1, i-1}(\omega)}{k_{1} \Delta_{N}},
\end{aligned}
$$

onde $\Delta_{1,0}=\Delta_{N+1, N}=1$. Assim a Eq. (D-2) assume a seguinte forma

$$
\left\langle v_{i} v_{l}\right\rangle=\frac{\gamma}{k_{1}^{2} \pi} \sum_{j=1, N} T_{j} \int_{-\infty}^{+\infty} \frac{\omega^{2}}{\left|\Delta_{N}\right|^{2}} d \omega \widetilde{\mathcal{A}}_{i j}(\omega) \widetilde{\mathcal{A}}_{l j}(-\omega) .
$$

Note que o termo destacado no integrando da Eq. (D-4) corresponde justamente ao que fora integrado na Eq. (C-16), o que permite uma grande simplificação no problema. Com o auxílio das relações construídas em (C-4) verifica-se que os subdeterminantes presentes na Eq. (D-3) são escritos como 


$$
\begin{aligned}
\Delta_{1, i-1} & =\delta_{i, 1}+\left[(b-a) \mathcal{R}_{2, i-1}-\mathcal{R}_{3, i-1}\right]\left(1-\delta_{i, 1}\right) \\
\Delta_{i+1, N} & =\delta_{i, N}+\left[(b-a) \mathcal{R}_{i+1, N-1}-\mathcal{R}_{i+1, N-2}\right]\left(1-\delta_{i, N}\right)
\end{aligned}
$$

onde $b$ e $a$ foram definidos no apêndice C. Fazendo uso agora da Eq. (C-13) pode se escrever a equação (D-5) da seguinte forma:

$$
\begin{aligned}
\Delta_{1, i-1} & =\delta_{i, 1}+\{(b-a) \sin [(i-1) \theta]-\sin [(i-2) \theta]\} \frac{\left(1-\delta_{i, 1}\right)}{\sin \theta} \\
\Delta_{i+1, N} & =\delta_{i, N}+\{(b-a) \sin [(N-i-1) \theta]-\sin [(N-i-2) \theta]\} \frac{\left(1-\delta_{i, N}\right)}{\sin \theta} .
\end{aligned}
$$

O fator que aparece no integrando da Eq. (D-4) multiplicando o termo que se encontra destacado nesta equação é obtido ao substituir as Eqs. (D-5) e (D-6) em (D-4), sendo este fator dado por

$$
\begin{aligned}
& \delta_{i, 1} \delta_{l, 1}{ }^{(\mathrm{i})}+\frac{\delta_{i, 1}\left(1-\delta_{l, 1}\right)}{\sin \theta}\left\{\left(b-a^{*}\right) \sin [(l-1) \theta]-\sin [(l-2) \theta]\right\}+ \\
& +\frac{\delta_{l, 1}\left(1-\delta_{i, 1}\right)}{\sin \theta}\{(b-a) \sin [(i-1) \theta]-\sin [(i-2) \theta]\}^{(i i i)}+ \\
& +\begin{array}{c}
\frac{\left(1-\delta_{i, 1}\right)\left(1-\delta_{l, 1}\right)}{\sin ^{2} \theta}\{(b-a) \sin [(i-1) \theta]-\sin [(i-2) \theta]\} \times \\
\times\left\{\left(b-a^{*}\right) \sin [(l-1) \theta]-\sin [(l-2) \theta]\right\}+
\end{array}+\delta_{i, N} \delta_{l, N}(\mathrm{v}) \\
& \left.+\frac{\delta_{i, N}\left(1-\delta_{l, N}\right)}{\sin \theta}\left\{\left(b-a^{*}\right) \sin [(N-l-1) \theta]-\sin [(N-l-2) \theta]\right\}\right\}^{(\mathrm{vi})}+ \\
& \left.+\frac{\delta_{l, N}\left(1-\delta_{i, N}\right)}{\sin \theta}\{(b-a) \sin [(N-i-1) \theta]-\sin [(N-i-2) \theta]\}\right\}^{\text {(vii) }}+
\end{aligned}
$$

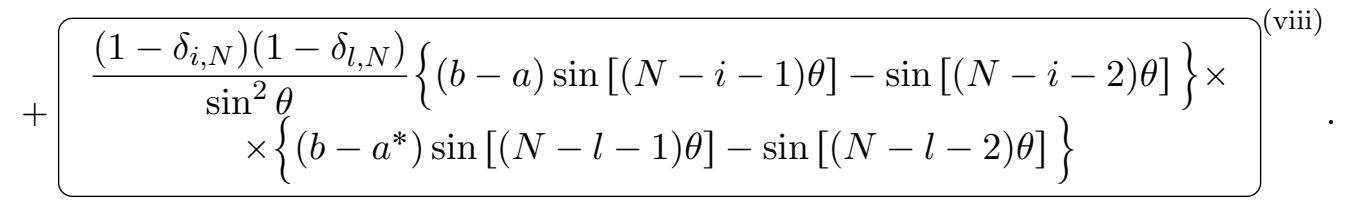

Agora faz-se a análise de cada um dos termos da Eq.(D-7) separadamente, visando computar a contribuição de cada um nas correlações entre partículas. Substituindo estes termos em (D-4) e fazendo uso do resultado encontrado em 
(C-22) obtém-se:

$$
\begin{aligned}
& \text { Contribuição do termo (i) }=\frac{k_{1}^{2}}{\pi m} \sum_{j=1, N} T_{j} \int_{0}^{\pi} \frac{\delta_{i, 1} \delta_{l, 1} \sin ^{2} \theta}{\Pi-\Theta \cos \theta} d \theta= \\
& =\sum_{j=1, N} T_{j} \frac{\delta_{i, 1} \delta_{l, 1} k_{1}^{2}}{m \Theta^{2}}\left(\Pi-\sqrt{\Pi^{2}-\Theta^{2}}\right) .
\end{aligned}
$$

Contribuição do termo (ii) $=$

$$
=\sum_{j=1, N} T_{j} \delta_{i, 1}\left(1-\delta_{l, 1}\right) \frac{k_{1}^{2}}{\pi m} \int_{0}^{\pi} \frac{\sin \theta\left\{\left(b-a^{*}\right) \sin [(l-1) \theta]-\sin [(l-2) \theta]\right\}}{\Pi-\Theta \cos \theta} d \theta .
$$

$$
\begin{aligned}
& \text { Contribuição do termo (iii) }= \\
& =\sum_{j=1, N} T_{j} \delta_{l, 1}\left(1-\delta_{i, 1}\right) \frac{k_{1}^{2}}{\pi m} \int_{0}^{\pi} \frac{\sin \theta\{(b-a) \sin [(i-1) \theta]-\sin [(i-2) \theta]\}}{\Pi-\Theta \cos \theta} d \theta .
\end{aligned}
$$

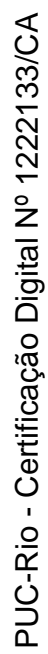

Contribuição do termo (iv) $=$

$$
\begin{aligned}
& =\sum_{j=1, N} T_{j} \frac{k_{1}^{2}}{\pi m} \int_{0}^{\pi} \frac{\left(1-\delta_{i, 1}\right)\left(1-\delta_{l, 1}\right)}{\Pi-\Theta \cos \theta}\{(b-a) \sin [(i-1) \theta]-\sin [(i-2) \theta]\} \times \\
& \times\left\{\left(b-a^{*}\right) \sin [(l-1) \theta]-\sin [(l-2) \theta]\right\} d \theta .
\end{aligned}
$$

$$
\begin{aligned}
& \text { Contribuição do termo }(\mathrm{v})=\frac{k_{1}^{2}}{\pi m} \sum_{j=1, N} T_{j} \int_{0}^{\pi} \frac{\delta_{i, N} \delta_{l, N} \sin ^{2} \theta}{\Pi-\Theta \cos \theta} d \theta= \\
& =\sum_{j=1, N} T_{j} \frac{\delta_{i, N} \delta_{l, N} k_{1}^{2}}{m \Theta^{2}}\left(\Pi-\sqrt{\Pi^{2}-\Theta^{2}}\right) .
\end{aligned}
$$

Contribuição do termo (vi) =

$=\sum_{j=1, N} T_{j} \delta_{l, N}\left(1-\delta_{i, N}\right) \frac{k_{1}^{2}}{\pi m} \int_{0}^{\pi} \frac{\sin \theta\left\{\left(b-a^{*}\right) \sin [(N-l-1) \theta]-\sin [(N-l-2) \theta]\right\}}{\Pi-\Theta \cos \theta} d \theta$.

Contribuição do termo (vii) $=$

$=\sum_{j=1, N} T_{j} \delta_{i, N}\left(1-\delta_{l, N}\right) \frac{k_{1}^{2}}{\pi m} \int_{0}^{\pi} \frac{\sin \theta\{(b-a) \sin [(N-i-1) \theta]-\sin [(N-i-2) \theta]\}}{\Pi-\Theta \cos \theta} d \theta$. 


$$
\begin{aligned}
& \text { Contribuição do termo (viii) }= \\
& =\sum_{j=1, N} T_{j} \frac{k_{1}^{2}}{\pi m} \int_{0}^{\pi} \frac{\left(1-\delta_{i, N}\right)\left(1-\delta_{l, N}\right)}{\Pi-\Theta \cos \theta}\{(b-a) \sin [(N-i-1) \theta]-\sin [(N-i-2) \theta]\} \times \\
& \times\left\{\left(b-a^{*}\right) \sin [(N-l-1) \theta]-\sin [(N-l-2) \theta]\right\} d \theta .
\end{aligned}
$$

Por conveniência mostra-se novamente as definições dos parâmetros que se encontram no integrando dados por:

$$
\begin{gathered}
b=2 \cos \theta \\
a=1+\frac{k-k^{\prime}-i \gamma \omega}{k_{1}}, \text { onde } \\
\omega=\sqrt{\frac{k+2 k_{1}(1-\cos \theta)}{m}} \\
\Theta=\frac{2 k_{1} \gamma^{2}}{m}+2 k_{1}\left(k_{1}+k-k^{\prime}\right) \\
\Pi=\frac{\left(2 k_{1}+k\right) \gamma^{2}}{m}+\left(k_{1}+k\right)^{2}+\left(k_{1}-k^{\prime}\right)^{2}-2 k^{\prime} k .
\end{gathered}
$$




\section{E \\ Desenvolvimento perturbativo do caso não linear}

A equação (4-2), pode ser escrita em notação indicial da seguinte forma:

$$
m \frac{d^{2} \mathbf{x}_{\mathbf{i}}}{d t^{2}}=\mathcal{D}_{i j} \mathbf{x}_{j}+\boldsymbol{\eta}+k_{3} f_{i}\left(\mathbf{x}_{\mathbf{p}}\right)
$$

onde o último termo da Eq. (E-1) representa uma função associada a interação cúbica entre as partículas.

Utilizando a informação de que a solução aproximada do problema pode ser obtida de forma perturbativa, faz-se uso das equações (E-1) e (4-5), de maneira que as equações de movimento em primeira ordem no parâmetro $\delta$ são escritas como:

$$
m \frac{d^{2} \mathbf{x}_{\mathbf{i}}{ }^{(0)}}{d t^{2}}+m \delta \frac{d^{2} \mathbf{x}_{\mathbf{i}}^{(1)}}{d t^{2}}=\mathcal{D}_{i j} \mathbf{x}_{j}^{(0)}+\delta \mathcal{D}_{i j} \mathbf{x}_{j}^{(1)}+\boldsymbol{\eta}+\delta \frac{k_{1}^{2}}{T_{N}} f_{i}\left(\mathbf{x}_{\mathbf{p}}{ }^{(0)}\right)
$$

identificando ordem a ordem em $\delta$ tem-se,

$$
\begin{gathered}
\mathcal{O}\left(\delta^{(0)}\right) \rightarrow m \frac{d^{2} \mathbf{x}_{\mathbf{i}}^{(0)}}{d t^{2}}=\mathcal{D}_{i j} \mathbf{x}_{j}^{(0)}+\boldsymbol{\eta}, \\
\mathcal{O}\left(\delta^{(1)}\right) \rightarrow m \frac{d^{2} \mathbf{x}_{\mathbf{i}}^{(1)}}{d t^{2}}=\mathcal{D}_{i j} \mathbf{x}_{j}^{(1)}+\frac{k_{1}^{2}}{T_{N}} f_{i}\left(\mathbf{x}_{\mathbf{l}}^{(0)}\right) .
\end{gathered}
$$

e

Escrevendo a transformada de Laplace da equação E-4 obtém-se:

$$
\begin{gathered}
\delta_{i j} m s^{2} \widetilde{\mathbf{x}}_{\mathbf{j}}^{(1)}=\mathcal{D}_{i j}(s) \widetilde{\mathbf{x}}_{j}^{(1)}+\frac{k_{1}^{2}}{T_{N}} \tilde{f}_{i}\left(\mathbf{x}_{\mathbf{p}}{ }^{(0)}\right) \rightarrow \\
\rightarrow\left(\delta_{i j} m s^{2}-\mathcal{D}_{i j}\right) \widetilde{\mathbf{x}}_{\mathbf{j}}^{(1)}=\frac{k_{1}^{2}}{T_{N}} \tilde{f}_{i}\left(\mathbf{x}_{\mathbf{p}}{ }^{(0)}\right)
\end{gathered}
$$

Lembrando que $\widetilde{\mathcal{A}}_{i j}$ é a matriz que diagonaliza as equações de movimento do caso linear, as posições das partículas em primeira ordem em $\delta$ são escritas como:

$$
\widetilde{\mathbf{x}}_{\mathbf{i}}^{(1)}=\frac{k_{1}^{2}}{T_{N}} \widetilde{\mathcal{A}}_{i j} \tilde{f}_{j}\left(\mathbf{x}_{\mathbf{p}}{ }^{(0)}\right)
$$

Antes de prosseguir, é conveniente definir aqui a forma da transformada de Laplace do termo cúbico presente no fluxo de calor, que é escrito da seguinte maneira, 


$$
\mathcal{L}\left[\left(x_{j}-x_{j+1}\right)^{3}\right](s)=\lim _{\alpha \rightarrow 0} \int_{\text {all space }} \prod_{m=3}^{5}\left\{\frac{d q_{m}}{(2 \pi)^{3}} \frac{\left[\widetilde{x_{j}}\left(i q_{m}+\alpha\right)-\widetilde{x}_{j+1}\left(i q_{m}+\alpha\right)\right]}{s-\left(i q_{3}+i q_{4}+i q_{5}+3 \alpha\right)}\right\}
$$

Para evitar uma notação indicial densa, analisa-se o fluxo de energia que flui da partícula 1 para 2, de forma que a equação (4-6) no espaço de Fourier-Laplace é escrita como,

$$
\begin{aligned}
& \langle J\rangle_{1 \rightarrow 2}=\lim _{z, \epsilon \rightarrow 0} \int_{\text {all space }} \frac{d q_{1}}{2 \pi} \frac{d q_{2}}{2 \pi} \frac{z\left(i q_{2}+\epsilon\right)}{z-\left(i q_{1}+i q_{2}+2 \epsilon\right)} \times \\
& \left\{\begin{array}{c}
\frac{k_{1}}{2} \underbrace{\left\langle\left[\widetilde{x}_{1}\left(i q_{1}+\epsilon\right)-\widetilde{x}_{2}\left(i q_{1}+\epsilon\right)\right]\left[\widetilde{x_{1}}\left(i q_{2}+\epsilon\right)+\widetilde{x}_{2}\left(i q_{2}+\epsilon\right)\right]\right\rangle}_{I}\}+
\end{array}\right.
\end{aligned}
$$

$$
\begin{aligned}
& +\lim _{z, \epsilon \rightarrow 0} \int_{\text {all space }} \frac{d q_{1}}{2 \pi} \frac{d q_{2}}{2 \pi} \frac{d q_{3}}{2 \pi} \frac{d q_{4}}{2 \pi} \frac{z\left(i q_{4}+\epsilon\right)}{z-\left(i q_{1}+i q_{2}+i q_{3}+i q_{4}+4 \epsilon\right)} \times \\
& \times\{\frac{k_{3}}{2} \lim _{\alpha \rightarrow 0} \underbrace{\int_{\text {all space }} \prod_{m=1}^{3} \frac{d q_{m}}{(2 \pi)^{3}}\left\langle\left[\widetilde{x_{1}}\left(i q_{m}+\alpha\right)-\widetilde{x}_{2}\left(i q_{m}+\alpha\right)\right]\left[\widetilde{x_{1}}\left(i q_{4}+\epsilon\right)+\widetilde{x}_{2}\left(i q_{4}+\epsilon\right)\right]\right\rangle}\}
\end{aligned}
$$

Note que E-8 é exata no âmbito da teoria de perturbação e independente da natureza dos banhos térmicos envolvidos. A partir desta parte faz-se o desenvolvimento dos termos $I$ e $I I$ separadamente, pois estes são extremamente extensos.

\section{E.1}

\section{Desenvolvimento do termo $I I$}

Como o interesse é desenvolver uma expansão pertubartiva em primeira ordem no parâmetro $\delta$, observe que na expressão $I I$ é necessário apenas levar em consideração no integrando a solução de ordem zero do problema, de maneira que,

$$
\widetilde{\mathbf{x}}_{i}\left(i q_{m}+\epsilon\right)=\widetilde{\mathbf{x}}_{i}^{(0)}\left(i q_{m}+\epsilon\right),
$$

onde os termos $\widetilde{\mathbf{x}}_{i}^{(0)}$ são escritos como:

$$
\widetilde{\mathbf{x}}_{i}^{(0)}\left(i q_{m}+\epsilon\right)=\widetilde{\mathcal{A}}_{\{i, 1\}}\left(i q_{m}+\epsilon\right) \widetilde{\eta}_{1}\left(i q_{m}+\epsilon\right)+\widetilde{\mathcal{A}}_{\{i, N\}}\left(i q_{m}+\epsilon\right) \widetilde{\eta}_{N}\left(i q_{m}+\epsilon\right) .
$$


Com o intuito de simplificar os cálculos a seguir, introduz-se as seguintes funções auxiliares:

$$
\begin{gathered}
\phi_{\{1,2\}}^{ \pm}\left(i q_{m}+\alpha\right)=\left[\widetilde{\mathcal{A}}_{\{1,1\}}\left(i q_{m}+\alpha\right) \pm \widetilde{\mathcal{A}}_{\{2,1\}}\left(i q_{m}+\alpha\right)\right] \widetilde{\eta}_{1}\left(i q_{m}+\alpha\right) \\
\omega_{\{1,2\}}^{ \pm}\left(i q_{m}+\alpha\right)=\left[\widetilde{\mathcal{A}}_{\{1, N\}}\left(i q_{m}+\alpha\right) \pm \widetilde{\mathcal{A}}_{\{2, N\}}\left(i q_{m}+\alpha\right)\right] \widetilde{\eta}_{N}\left(i q_{m}+\alpha\right) \\
\chi_{\{1,2\}}^{ \pm}\left(i q_{m}+\alpha\right)=\widetilde{\mathcal{A}}_{\{1,1\}}\left(i q_{m}+\alpha\right) \pm \widetilde{\mathcal{A}}_{\{2,1\}}\left(i q_{m}+\alpha\right) \\
\Omega_{\{1,2\}}^{ \pm}\left(i q_{m}+\alpha\right)=\widetilde{\mathcal{A}}_{\{1, N\}}\left(i q_{m}+\alpha\right) \pm \widetilde{\mathcal{A}}_{\{2, N\}}\left(i q_{m}+\alpha\right) \\
\phi_{\{1,2\}}^{ \pm}\left(i q_{m}+\alpha\right)=\chi_{\{1,2\}}^{ \pm}\left(i q_{m}+\alpha\right) \widetilde{\eta}_{1}\left(i q_{m}+\alpha\right) \\
\omega_{\{1,2\}}^{ \pm}\left(i q_{m}+\alpha\right)=\Omega_{\{1,2\}}^{ \pm}\left(i q_{m}+\alpha\right) \widetilde{\eta}_{N}\left(i q_{m}+\alpha\right)
\end{gathered}
$$

Expandindo o núcleo do numerador do termo $I I$ encontra-se,

$$
\begin{aligned}
& \left\langle\left\{\prod_{m=3}^{5}\left[\phi_{\{1,2\}}^{-}\left(i q_{m}+\alpha\right)\right]+\phi_{\{1,2\}}^{-}\left(i q_{3}+\alpha\right) \phi_{\{1,2\}}^{-}\left(i q_{5}+\alpha\right) \omega_{\{1,2\}}^{-}\left(i q_{4}+\alpha\right)+\right.\right. \\
& +\phi_{\{1,2\}}^{-}\left(i q_{4}+\alpha\right) \phi_{\{1,2\}}^{-}\left(i q_{5}+\alpha\right) \omega_{\{1,2\}}^{-}\left(i q_{3}+\alpha\right)+\phi_{\{1,2\}}^{-}\left(i q_{5}+\alpha\right) \omega_{\{1,2\}}^{-}\left(i q_{3}+\alpha\right) \omega_{\{1,2\}}^{-}\left(i q_{4}+\alpha\right)+ \\
& +\phi_{\{1,2\}}^{-}\left(i q_{3}+\alpha\right) \phi_{\{1,2\}}^{-}\left(i q_{4}+\alpha\right) \omega_{\{1,2\}}^{-}\left(i q_{5}+\alpha\right)+\phi_{\{1,2\}}^{-}\left(i q_{3}+\alpha\right) \omega_{\{1,2\}}^{-}\left(i q_{4}+\alpha\right) \omega_{\{1,2\}}^{-}\left(i q_{5}+\alpha\right)+ \\
& \left.+\phi_{\{1,2\}}^{-}\left(i q_{4}+\alpha\right) \omega_{\{1,2\}}^{-}\left(i q_{3}+\alpha\right) \omega_{\{1,2\}}^{-}\left(i q_{5}+\alpha\right)+\prod_{m=3}^{5}\left[\omega_{\{1,2\}}^{-}\left(i q_{m}+\alpha\right)\right]\right\} \times \\
& \left.\times\left[\phi_{\{1,2\}}^{+}\left(i q_{2}+\epsilon\right)+\omega_{\{1,2\}}^{+}\left(i q_{2}+\epsilon\right)\right]\left(i q_{2}+\epsilon\right)\right\rangle
\end{aligned}
$$

Ao analisar a Eq. (E-14) é fácil de se notar que quando esta é multiplicada por $\left[\phi_{\{1,2\}}^{-}\left(i q_{2}+\epsilon\right)+\omega_{\{1,2\}}^{-}\left(i q_{2}+\epsilon\right)\right]$, os termos gerados que possuem um número ímpar de $\omega$ ou $\phi$, não contribuem devido ao ruído associado aos reservatórios ser do tipo gaussiano branco, o que leva a (E-14) em: 


$$
\begin{aligned}
& {\left[\left\langle\prod_{m=3}^{5} \phi_{\{1,2\}}^{-}\left(i q_{m}+\alpha\right) \phi_{\{1,2\}}^{+}\left(i q_{2}+\epsilon\right)+\right.\right.} \\
& +\phi_{\{1,2\}}^{+}\left(i q_{2}+\epsilon\right) \phi_{\{1,2\}}^{-}\left(i q_{5}+\alpha\right) \omega_{\{1,2\}}^{-}\left(i q_{3}+\alpha\right) \omega_{\{1,2\}}^{-}\left(i q_{4}+\alpha\right)+ \\
& +\phi_{\{1,2\}}^{+}\left(i q_{2}+\epsilon\right) \phi_{\{1,2\}}^{-}\left(i q_{3}+\alpha\right) \omega_{\{1,2\}}^{-}\left(i q_{4}+\alpha\right) \omega_{\{1,2\}}^{-}\left(i q_{5}+\alpha\right)+ \\
& \left.\left.+\phi_{\{1,2\}}^{+}\left(i q_{2}+\epsilon\right) \phi_{\{1,2\}}^{-}\left(i q_{4}+\alpha\right) \omega_{\{1,2\}}^{-}\left(i q_{3}+\alpha\right) \omega_{\{1,2\}}^{-}\left(i q_{5}+\alpha\right)\right\rangle\right]\left(i q_{2}+\epsilon\right)+ \\
& +\left[\left\langle\prod_{m=3}^{5} \omega_{\{1,2\}}^{-}\left(i q_{m}+\alpha\right) \omega_{\{1,2\}}^{+}\left(i q_{2}+\epsilon\right)+\right.\right. \\
& \quad \phi_{\{1,2\}}^{-}\left(i q_{3}+\alpha\right) \phi_{\{1,2\}}^{-}\left(i q_{5}+\alpha\right) \omega_{\{1,2\}}^{+}\left(i q_{2}+\epsilon\right) \omega_{\{1,2\}}^{-}\left(i q_{4}+\alpha\right) \\
& +\phi_{\{1,2\}}^{-}\left(i q_{4}+\alpha\right) \phi_{\{1,2\}}^{-}\left(i q_{5}+\alpha\right) \omega_{\{1,2\}}^{+}\left(i q_{2}+\epsilon\right) \omega_{\{1,2\}}^{-}\left(i q_{4}+\alpha\right) \\
& \left.\left.+\phi_{\{1,2\}}^{-}\left(i q_{3}+\alpha\right) \phi_{\{1,2\}}^{-}\left(i q_{4}+\alpha\right) \omega_{\{1,2\}}^{+}\left(i q_{2}+\epsilon\right) \omega_{\{1,2\}}^{-}\left(i q_{5}+\alpha\right)\right\rangle\right]\left(i q_{2}+\epsilon\right) \quad(\mathrm{E}-15
\end{aligned}
$$

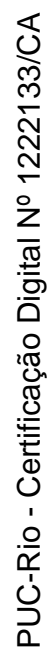

Agora que a expressão (E-15) apresenta uma versão mais clara do termo II, é possível avaliar as médias no respectivo integrando. Para resolver a Eq. (E-15) pode se aplicar o teorema de Wick, (ver apêndice F), aos termos resultantes dos produtos da expressão ou analisar esta equação através da contagem de diagramas associados às médias presentes. Notando as simetrias presentes no desenvolvimento da equação (E-15), a contribuição final deste termo cúbico pode ser escrita como,

$$
\begin{aligned}
& I I=\frac{3 k_{3}}{2}(2 \gamma)^{2} \times \\
& \times\left\{T_{1}^{2} \int_{-\infty}^{\infty} \frac{d q_{1}}{2 \pi}\left(i q_{1}+\epsilon\right) \chi_{\{1,2\}}^{+}\left(i q_{1}+\epsilon\right) \chi_{\{1,2\}}^{-}\left(-i q_{1}-\epsilon\right) \times\right. \\
& \times \int_{-\infty}^{\infty} \frac{d q_{2}}{2 \pi} \chi_{\{1,2\}}^{-}\left(i q_{2}+\epsilon\right) \chi_{\{1,2\}}^{-}\left(-i q_{2}-\epsilon\right)+ \\
& T_{1} T_{N}\left[\int_{-\infty}^{\infty} \frac{d q_{1}}{2 \pi}\left(i q_{1}+\epsilon\right) \chi_{\{1,2\}}^{+}\left(i q_{1}+\epsilon\right) \chi_{\{1,2\}}^{-}\left(-i q_{1}-\epsilon\right) \times\right. \\
& \times \int_{-\infty}^{\infty} \frac{d q_{2}}{2 \pi} \Omega_{\{1,2\}}^{-}\left(i q_{2}+\epsilon\right) \Omega_{\{1,2\}}^{-}\left(-i q_{2}-\epsilon\right)+ \\
& +\int_{-\infty}^{\infty} \frac{d q_{1}}{2 \pi} \chi_{\{1,2\}}^{-}\left(i q_{1}+\epsilon\right) \chi_{\{1,2\}}^{-}\left(-i q_{1}-\epsilon\right) \times \\
& \left.\times \int_{-\infty}^{\infty} \frac{d q_{2}}{2 \pi}\left(i q_{2}+\epsilon\right) \Omega_{\{1,2\}}^{+}\left(i q_{2}+\epsilon\right) \Omega_{\{1,2\}}^{-}\left(-i q_{2}-\epsilon\right)\right]+ \\
& +T_{N}^{2} \int_{-\infty}^{\infty} \frac{d q_{1}}{2 \pi}\left(i q_{1}+\epsilon\right) \Omega_{\{1,2\}}^{+}\left(i q_{1}+\epsilon\right) \Omega_{\{1,2\}}^{-}\left(-i q_{1}-\epsilon\right) \times \\
& \left.\times \int_{-\infty}^{\infty} \frac{d q_{2}}{2 \pi} \Omega_{\{1,2\}}^{-}\left(i q_{2}+\epsilon\right) \Omega_{\{1,2\}}^{-}\left(-i q_{2}-\epsilon\right)\right\}
\end{aligned}
$$




\section{E.2}

\section{Desenvolvimento do termo $I$}

Expandindo o númerador da expressão $I$ tem-se:

$$
\left\langle\left[\widetilde{x_{1}}\left(i q_{1}+\epsilon\right)-\widetilde{x}_{2}\left(i q_{1}+\epsilon\right)\right]\left[\widetilde{x_{1}}\left(i q_{2}+\epsilon\right)+\widetilde{x}_{2}\left(i q_{2}+\epsilon\right)\right]\right\rangle
$$

Para desenvolver a expansão em ordem 1 deste termo, é necessário considerar apenas os fatores lineares em $\delta$ na expansão perturbativa das posições das partículas, que é dada por:

$$
\widetilde{\mathbf{x}}_{i}\left(i q_{m}+\epsilon\right)=\widetilde{\mathbf{x}}_{i}^{(0)}\left(i q_{m}+\epsilon\right)+\delta \widetilde{\mathbf{x}}_{i}^{(1)}\left(i q_{m}+\epsilon\right)
$$

Inserindo (E-18) em (E-17) obtém-se:

$$
\begin{aligned}
& \left\langle\left\{\widetilde{\mathbf{x}}_{1}^{(0)}\left(i q_{1}+\epsilon\right)-\widetilde{\mathbf{x}}_{2}^{(0)}\left(i q_{1}+\epsilon\right)+\delta\left[\widetilde{\mathbf{x}}_{1}^{(1)}\left(i q_{1}+\epsilon\right)-\widetilde{\mathbf{x}}_{2}^{(1)}\left(i q_{1}+\epsilon\right)\right]\right\} \times\right. \\
& \left.\times\left\{\widetilde{\mathbf{x}}_{1}^{(0)}\left(i q_{2}+\epsilon\right)+\widetilde{\mathbf{x}}_{2}^{(0)}\left(i q_{2}+\epsilon\right)+\delta\left[\widetilde{\mathbf{x}}_{1}^{(1)}\left(i q_{2}+\epsilon\right)+\widetilde{\mathbf{x}}_{2}^{(1)}\left(i q_{2}+\epsilon\right)\right]\right\}\right\rangle\left(i q_{2}+\epsilon\right)= \\
& =\left\langle\left\{\phi_{\{1,2\}}^{-}\left(i q_{1}+\alpha\right)+\omega_{\{1,2\}}^{-}\left(i q_{1}+\alpha\right)+\delta\left[\widetilde{\mathbf{x}}_{1}^{(1)}\left(i q_{1}+\epsilon\right)-\widetilde{\mathbf{x}}_{2}^{(1)}\left(i q_{1}+\epsilon\right)\right]\right\} \times\right. \\
& \left.\times\left\{\phi_{\{1,2\}}^{+}\left(i q_{2}+\epsilon\right)+\omega_{\{1,2\}}^{+}\left(i q_{2}+\epsilon\right)+\delta\left[\widetilde{\mathbf{x}}_{1}^{(1)}\left(i q_{2}+\epsilon\right)+\widetilde{\mathbf{x}}_{2}^{(1)}\left(i q_{2}+\epsilon\right)\right]\right\}\right\rangle\left(i q_{2}+\epsilon\right)
\end{aligned}
$$

Para prosseguir é preciso escrever explícitamente à equação E-6 para cada partícula. A forma das função $\widetilde{f}_{j}\left(\widetilde{\mathbf{x}}_{p}^{(0)}\right)$ depende da posição da partícula na cadeia, visto que a interação efetiva dos elementos das extremidades, $(j=$ $1, N)$, é diferente dos elementos do volume. Para as partículas das extremidades 
tem-se que as funções $\widetilde{f}_{1, N}\left(\widetilde{\mathbf{x}}_{p}^{(0)}\right)$ são escritas como,

$$
\begin{aligned}
& \widetilde{f}_{1}\left(\widetilde{\mathbf{x}}_{p}^{(0)}\right) \equiv \tilde{f}_{1}\left(i q_{\beta}+\epsilon\right)=-\lim _{\alpha \rightarrow 0} \int_{\text {all space }} \prod_{m=3}^{5}\left\{\frac{d q_{m}}{(2 \pi)^{3}} \frac{\left[\widetilde{x_{1}}\left(i q_{m}+\alpha\right)-\widetilde{x}_{2}\left(i q_{m}+\alpha\right)\right]}{i q_{\beta}+\epsilon-\left(i q_{3}+i q_{4}+i q_{5}+3 \alpha\right)}\right\}= \\
& =-\lim _{\alpha \rightarrow 0} \int_{\text {all space }} \prod_{m=3}^{5} \frac{d q_{m}}{(2 \pi)^{3}} \frac{\left[\widetilde{\mathbf{x}}_{1}^{(0)}\left(i q_{m}+\epsilon\right)-\widetilde{\mathbf{x}}_{2}^{(0)}\left(i q_{m}+\epsilon\right)\right]}{i q_{\beta}+\epsilon-\left(i q_{3}+i q_{4}+i q_{5}+3 \alpha\right)} \\
& =-\lim _{\alpha \rightarrow 0} \int_{\text {all space }} \prod_{m=3}^{5} \frac{d q_{m}}{(2 \pi)^{3}} \frac{\phi_{\{1,2\}}^{-}\left(i q_{m}+\alpha\right)+\omega_{\{1,2\}}^{-}\left(i q_{m}+\alpha\right)}{i q_{\beta}+\epsilon-\left(i q_{3}+i q_{4}+i q_{5}+3 \alpha\right)}= \\
& =-\int_{\text {all space }} \prod_{m=3}^{5} \frac{d q_{m}}{(2 \pi)^{3}} \times \frac{1}{i q_{\beta}+\epsilon-\left(i q_{3}+i q_{4}+i q_{5}+3 \alpha\right)} \times \\
& \times\left\{\prod_{m=3}^{5}\left[\phi_{\{1,2\}}^{-}\left(i q_{m}+\alpha\right)\right]+\phi_{\{1,2\}}^{-}\left(i q_{3}+\alpha\right) \phi_{\{1,2\}}^{-}\left(i q_{5}+\alpha\right) \omega_{\{1,2\}}^{-}\left(i q_{4}+\alpha\right)+\right. \\
& +\phi_{\{1,2\}}^{-}\left(i q_{4}+\alpha\right) \phi_{\{1,2\}}^{-}\left(i q_{5}+\alpha\right) \omega_{\{1,2\}}^{-}\left(i q_{3}+\alpha\right)+\phi_{\{1,2\}}^{-}\left(i q_{5}+\alpha\right) \omega_{\{1,2\}}^{-}\left(i q_{3}+\alpha\right) \omega_{\{1,2\}}^{-}\left(i q_{4}+\alpha\right)+ \\
& +\phi_{\{1,2\}}^{-}\left(i q_{3}+\alpha\right) \phi_{\{1,2\}}^{-}\left(i q_{4}+\alpha\right) \omega_{\{1,2\}}^{-}\left(i q_{5}+\alpha\right)+\phi_{\{1,2\}}^{-}\left(i q_{3}+\alpha\right) \omega_{\{1,2\}}^{-}\left(i q_{4}+\alpha\right) \omega_{\{1,2\}}^{-}\left(i q_{5}+\alpha\right)+ \\
& \left.+\phi_{\{1,2\}}^{-}\left(i q_{4}+\alpha\right) \omega_{\{1,2\}}^{-}\left(i q_{3}+\alpha\right) \omega_{\{1,2\}}^{-}\left(i q_{5}+\alpha\right)+\prod_{m=3}^{5}\left[\omega_{\{1,2\}}^{-}\left(i q_{m}+\alpha\right)\right]\right\}
\end{aligned}
$$

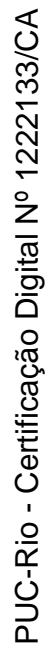

$$
\begin{aligned}
& \tilde{f}_{N}\left(\widetilde{\mathbf{x}}_{p}^{(0)}\right) \equiv \widetilde{f}_{N}\left(i q_{\beta}+\epsilon\right)=-\lim _{\alpha \rightarrow 0} \int_{\text {all space }} \prod_{m=3}^{5}\left\{\frac{d q_{m}}{(2 \pi)^{3}} \frac{\left[\widetilde{x_{N}}\left(i q_{m}+\alpha\right)-\widetilde{x}_{N-1}\left(i q_{m}+\alpha\right)\right]}{i q_{\beta}+\epsilon-\left(i q_{3}+i q_{4}+i q_{5}+3 \alpha\right)}\right\}= \\
& =-\lim _{\alpha \rightarrow 0} \int_{\text {all space }} \prod_{m=3}^{5} \frac{d q_{m}}{(2 \pi)^{3}} \frac{\left[\widetilde{\mathbf{x}}_{N}^{(0)}\left(i q_{m}+\epsilon\right)-\widetilde{\mathbf{x}}_{N-1}^{(0)}\left(i q_{m}+\epsilon\right)\right]}{i q_{\beta}+\epsilon-\left(i q_{3}+i q_{4}+i q_{5}+3 \alpha\right)} \\
& =+\lim _{\alpha \rightarrow 0} \int_{\text {all space }} \prod_{m=3}^{5} \frac{d q_{m}}{(2 \pi)^{3}} \frac{\phi_{\{N-1, N\}}^{-}\left(i q_{m}+\alpha\right)+\omega_{\{N-1, N\}}^{-}\left(i q_{m}+\alpha\right)}{i q_{\beta}+\epsilon-\left(i q_{3}+i q_{4}+i q_{5}+3 \alpha\right)}= \\
& =\int_{\text {all space }} \prod_{m=3}^{5} \frac{d q_{m}}{(2 \pi)^{3}} \times \frac{1}{i q_{\beta}+\epsilon-\left(i q_{3}+i q_{4}+i q_{5}+3 \alpha\right)} \times \\
& \times\left\{\prod_{m=3}^{5}\left[\phi_{\{N-1, N\}}^{-}\left(i q_{m}+\alpha\right)\right]+\phi_{\{N-1, N\}}^{-}\left(i q_{3}+\alpha\right) \phi_{\{N-1, N\}}^{-}\left(i q_{5}+\alpha\right) \omega_{\{N-1, N\}}^{-}\left(i q_{4}+\alpha\right)+\right. \\
& +\phi_{\{N-1, N\}}^{-}\left(i q_{4}+\alpha\right) \phi_{\{N-1, N\}}^{-}\left(i q_{5}+\alpha\right) \omega_{\{N-1, N\}}^{-}\left(i q_{3}+\alpha\right)+ \\
& +\phi_{\{N-1, N\}}^{-}\left(i q_{5}+\alpha\right) \omega_{\{N-1, N\}}^{-}\left(i q_{3}+\alpha\right) \omega_{\{N-1, N\}}^{-}\left(i q_{4}+\alpha\right)+ \\
& +\phi_{\{N-1, N\}}^{-}\left(i q_{3}+\alpha\right) \phi_{\{N-1, N\}}^{-}\left(i q_{4}+\alpha\right) \omega_{\{N-1, N\}}^{-}\left(i q_{5}+\alpha\right)+ \\
& +\phi_{\{N-1, N\}}^{-}\left(i q_{3}+\alpha\right) \omega_{\{N-1, N\}}^{-}\left(i q_{4}+\alpha\right) \omega_{\{N-1, N\}}^{-}\left(i q_{5}+\alpha\right)+ \\
& \left.+\phi_{\{N-1, N\}}^{-}\left(i q_{4}+\alpha\right) \omega_{\{N-1, N\}}^{-}\left(i q_{3}+\alpha\right) \omega_{\{N-1, N\}}^{-}\left(i q_{5}+\alpha\right)+\prod_{m=3}^{5}\left[\omega_{\{N-1, N\}}^{-}\left(i q_{m}+\alpha\right)\right]\right\} .
\end{aligned}
$$

Observe que a mudança no sinal negativo na Eq. (E-21) foi feita intencionalmente, de forma a manter consistência com a notação adotada 
em (E-11). A função $\tilde{f}_{j}\left(i q_{\beta}+\epsilon\right)$, considerando $j \neq 1, N$, é dada por:

$$
\begin{aligned}
& \tilde{f}_{j}\left(i q_{\beta}+\epsilon\right)=-\left\{\lim _{\alpha \rightarrow 0} \int_{\text {all space }} \prod_{m=3}^{5} \frac{d q_{m}}{(2 \pi)^{3}} \frac{\left[\widetilde{x_{j}}\left(i q_{m}+\alpha\right)-\widetilde{x}_{j+1}\left(i q_{m}+\alpha\right)\right]}{\left(i q_{\beta}+\epsilon\right)-\left(i q_{3}+i q_{4}+i q_{5}+3 \alpha\right)}+\right. \\
& \left.+\lim _{\alpha \rightarrow 0} \int_{\text {all space }} \prod_{m=3}^{5} \frac{d q_{m}}{(2 \pi)^{3}} \frac{\left[\widetilde{x_{j}}\left(i q_{m}+\alpha\right)-\widetilde{x}_{j-1}\left(i q_{m}+\alpha\right)\right]}{\left(i q_{\beta}+\epsilon\right)-\left(i q_{3}+i q_{4}+i q_{5}+3 \alpha\right)}\right\}= \\
& \lim _{\alpha \rightarrow 0} \int_{\text {all space }} \prod_{m=3}^{5} \frac{d q_{m}}{(2 \pi)^{3}} \frac{\left[\widetilde{x}_{j-1}\left(i q_{m}+\alpha\right)-\tilde{x}_{j}\left(i q_{m}+\alpha\right)\right]}{\left(i q_{\beta}+\epsilon\right)-\left(i q_{3}+i q_{4}+i q_{5}+3 \alpha\right)}+ \\
& -\lim _{\alpha \rightarrow 0} \int_{\text {all space }} \prod_{m=3}^{5} \frac{d q_{m}}{(2 \pi)^{3}} \frac{\left[\widetilde{x_{j}}\left(i q_{m}+\alpha\right)-\widetilde{x}_{j+1}\left(i q_{m}+\alpha\right)\right]}{\left(i q_{\beta}+\epsilon\right)-\left(i q_{3}+i q_{4}+i q_{5}+3 \alpha\right)},
\end{aligned}
$$

nota-se que a mudança no sinal negativo foi feita novamente devido ao mesmo argumento apresentado anteriormente. No desenvolvimento da Eq. (E-22) utiliza o mesmo procedimento matemático envolvido em (E-20) e (E-21), de maneira que não é necessário apresentar a sua expansão explicitamente. Voltando agora para (E-19) e fazendo uso da Eq. (E-6), nota-se que as diferenças e as somas entre as posições das partículas, em primeira ordem, são escritas como:

$$
\begin{aligned}
& \widetilde{x}_{1}^{(1)}\left(i q_{1}+\alpha\right)-\widetilde{x}_{2}^{(1)}\left(i q_{1}+\alpha\right)=\frac{k_{1}^{2}}{T_{N}}\left\{\left[\widetilde{\mathcal{A}}_{\{1,1\}}\left(i q_{1}+\alpha\right)-\widetilde{\mathcal{A}}_{\{2,1\}}\left(i q_{1}+\alpha\right)\right] \tilde{f}_{1}\left(i q_{1}+\epsilon\right)+\right. \\
& +\left[\widetilde{\mathcal{A}}_{\{1,2\}}\left(i q_{1}+\alpha\right)-\widetilde{\mathcal{A}}_{\{2,2\}}\left(i q_{1}+\alpha\right)\right] \widetilde{f}_{2}\left(i q_{1}+\epsilon\right)+\ldots+ \\
& \left.+\ldots+\left[\widetilde{\mathcal{A}}_{\{1, N\}}\left(i q_{1}+\alpha\right)-\widetilde{\mathcal{A}}_{\{2, N\}}\left(i q_{1}+\alpha\right)\right] \widetilde{f}_{N}\left(i q_{1}+\epsilon\right)\right\}
\end{aligned}
$$

e

$$
\begin{aligned}
& \widetilde{x}_{1}^{(1)}\left(i q_{1}+\alpha\right)+\widetilde{x}_{2}^{(1)}\left(i q_{1}+\alpha\right)=\frac{k_{1}^{2}}{T_{N}}\left\{\left[\widetilde{\mathcal{A}}_{\{1,1\}}\left(i q_{1}+\alpha\right)+\widetilde{\mathcal{A}}_{\{2,1\}}\left(i q_{1}+\alpha\right)\right] \tilde{f}_{1}\left(i q_{1}+\epsilon\right)+\right. \\
& +\left[\widetilde{\mathcal{A}}_{\{1,2\}}\left(i q_{1}+\alpha\right)+\widetilde{\mathcal{A}}_{\{2,2\}}\left(i q_{1}+\alpha\right)\right] \widetilde{f}_{2}\left(i q_{1}+\epsilon\right)+\ldots+ \\
& \left.+\ldots+\left[\widetilde{\mathcal{A}}_{\{1, N\}}\left(i q_{1}+\alpha\right)+\widetilde{\mathcal{A}}_{\{2, N\}}\left(i q_{1}+\alpha\right)\right] \widetilde{f}_{N}\left(i q_{1}+\epsilon\right)\right\} .
\end{aligned}
$$

Para simplificar as duas últimas equações e os passos seguintes, define-se duas funções auxiliares dadas por,

$$
\begin{aligned}
\Gamma_{\{1, j\}}\left(i q_{1}+\epsilon\right) & =\widetilde{\mathcal{A}}_{\{1, j\}}\left(i q_{1}+\alpha\right)-\widetilde{\mathcal{A}}_{\{2, j\}}\left(i q_{1}+\alpha\right) \\
\Lambda_{\{1, j\}}\left(i q_{2}+\epsilon\right) & =\widetilde{\mathcal{A}}_{\{1, j\}}\left(i q_{2}+\alpha\right)+\widetilde{\mathcal{A}}_{\{2, j\}}\left(i q_{2}+\alpha\right) .
\end{aligned}
$$


Assim é possível reescrever a equação E-19 como:

$$
\begin{aligned}
& \left\langle\left\{\phi_{\{1,2\}}^{-}\left(i q_{1}+\alpha\right)+\omega_{\{1,2\}}^{-}\left(i q_{1}+\alpha\right)+\frac{\delta k_{1}^{2}}{T_{N}}\left[\Gamma_{\{1, j\}}\left(i q_{1}+\epsilon\right) \tilde{f}_{j}\left(i q_{1}+\epsilon\right)\right]\right\} \times\right. \\
& \left.\times\left\{\phi_{\{1,2\}}^{+}\left(i q_{2}+\epsilon\right)+\omega_{\{1,2\}}^{+}\left(i q_{2}+\epsilon\right)+\frac{\delta k_{1}^{2}}{T_{N}}\left[\Lambda_{\{1, k\}}\left(i q_{2}+\epsilon\right) \tilde{f}_{k}\left(i q_{2}+\epsilon\right)\right]\right\}\right\rangle\left(i q_{2}+\epsilon\right) .
\end{aligned}
$$

Vale frisar aqui que na Eq. (E-26) utilizou-se a notação para soma de Einstein. Analisando a equação (E-26), nota-se que os termos em ordem zero no parâmetro $\delta$ quando multiplicados entre si, dão a contribuição associada do caso puramente harmônico. O produto dos termos lineares em $\delta$, levam a correções de segunda ordem, logo estes termos podem ser negligenciados. Com base nestas informações, os termos que realmente importam são:

$$
\begin{aligned}
& \left.\left\langle\left[\phi_{\{1,2\}}^{-}\left(i q_{1}+\alpha\right)+\omega_{\{1,2\}}^{-}\left(i q_{1}+\alpha\right)\right] \tilde{f}_{k}\left(i q_{2}+\epsilon\right)\right)\right\rangle\left(i q_{2}+\epsilon\right) \frac{\delta k_{1}^{2}}{T_{N}} \Lambda_{\{1, k\}}\left(i q_{2}+\epsilon\right)+ \\
& \left.+\left\langle\left[\phi_{\{1,2\}}^{+}\left(i q_{2}+\alpha\right)+\omega_{\{1,2\}}^{+}\left(i q_{2}+\alpha\right)\right] \tilde{f}_{k}\left(i q_{1}+\epsilon\right)\right)\right\rangle\left(i q_{2}+\epsilon\right) \frac{\delta k_{1}^{2}}{T_{N}} \Gamma_{\{1, k\}}\left(i q_{1}+\epsilon\right)
\end{aligned}
$$

Observe que na Eq. (E-27), as funções ${\widetilde{f_{k}}}_{k}^{\prime} s$ são mantidas dentro do fator que toma a média sobre as realizações do ruído, pois elas contêm informação estocástica em sua forma explícita. É simples de se notar que a expansão envolvendo $\tilde{f}_{k}^{\prime}$ s em (E-27) é muito similar, no que diz respeito a cálculo de médias, com as expansões que surgiram em (E-15), diferindo apenas por alguns índices. Após um tanto de álgebra e fazendo $\frac{\delta k_{1}^{2}}{T_{N}} \equiv k_{3}$, tem-se que o desenvolvimento do termo (E-27) quando combinado com $I$, resulta em: 


$$
\begin{aligned}
& I=\lim _{\epsilon, \alpha \rightarrow 0} 3 k_{3}(2 \gamma)^{2} \times \\
& \times\left\{\sum _ { j = 2 } ^ { N } \left[T_{1}^{2} \int_{-\infty}^{\infty} \frac{d q_{1}}{2 \pi}\left(i q_{1}+\epsilon\right) \Lambda_{\{1, j\}}\left(i q_{1}+\epsilon\right) \chi_{\{j-1, j\}}^{-}\left(i q_{1}+\epsilon\right) \chi_{\{1,2\}}^{-}\left(-i q_{1}-\epsilon\right) \times\right.\right. \\
& \times \int_{-\infty}^{\infty} \frac{d q_{2}}{2 \pi} \chi_{\{j-1, j\}}^{-}\left(i q_{2}+\alpha\right) \chi_{\{j-1, j\}}^{-}\left(-i q_{2}-\alpha\right)+ \\
& +T_{1} T_{N}\left(\int_{-\infty}^{\infty} \frac{d q_{1}}{2 \pi}\left(i q_{1}+\epsilon\right) \Lambda_{\{1, j\}}\left(i q_{1}+\epsilon\right) \chi_{\{j-1, j\}}^{-}\left(i q_{1}+\epsilon\right) \chi_{\{1,2\}}^{-}\left(-i q_{1}-\epsilon\right) \times\right. \\
& \times \int_{-\infty}^{\infty} \frac{d q_{2}}{2 \pi} \Omega_{\{j, j-1\}}^{-}\left(i q_{2}+\alpha\right) \Omega_{\{j-1, j\}}^{-}\left(-i q_{2}-\alpha\right)+ \\
& +\int_{-\infty}^{\infty} \frac{d q_{1}}{2 \pi}\left(i q_{1}+\epsilon\right) \Lambda_{\{1, j\}}\left(i q_{1}+\epsilon\right) \Omega_{\{j-1, j\}}^{-}\left(i q_{1}+\epsilon\right) \Omega_{\{1,2\}}^{-}\left(-i q_{1}-\epsilon\right) \times \\
& \left.\times \int_{-\infty}^{\infty} \frac{d q_{2}}{2 \pi} \chi_{\{j-1, j\}}^{-}\left(i q_{2}+\alpha\right) \chi_{\{j-1, j\}}^{-}\left(-i q_{2}-\alpha\right)\right)+ \\
& +T_{N}^{2} \int_{-\infty}^{\infty} \frac{d q_{1}}{2 \pi}\left(i q_{1}+\epsilon\right) \Lambda_{\{1, j\}}\left(i q_{1}+\epsilon\right) \Omega_{\{j-1, j\}}^{-}\left(i q_{1}+\epsilon\right) \Omega_{\{1,2\}}^{-}\left(-i q_{1}-\epsilon\right) \times \\
& \left.\times \int_{-\infty}^{\infty} \frac{d q_{2}}{2 \pi} \Omega_{\{j-1, j\}}^{-}\left(i q_{2}+\alpha\right) \Omega_{\{j-1, j\}}^{-}\left(-i q_{2}-\alpha\right)\right]+ \\
& -\sum_{j=1}^{N-1}\left[T_{1}^{2} \int_{-\infty}^{\infty} \frac{d q_{1}}{2 \pi}\left(i q_{1}+\epsilon\right) \Lambda_{\{1, j\}}\left(i q_{1}+\epsilon\right) \chi_{\{j, j+1\}}^{-}\left(i q_{1}+\epsilon\right) \chi_{\{1,2\}}^{-}\left(-i q_{1}-\epsilon\right) \times\right. \\
& \times \int_{-\infty}^{\infty} \frac{d q_{2}}{2 \pi} \chi_{\{j, j+1\}}^{-}\left(i q_{2}+\alpha\right) \chi_{\{j, j+1\}}^{-}\left(-i q_{2}-\alpha\right)+ \\
& +T_{1} T_{N}\left(\int_{-\infty}^{\infty} \frac{d q_{1}}{2 \pi}\left(i q_{1}+\epsilon\right) \Lambda_{\{1, j\}}\left(i q_{1}+\epsilon\right) \chi_{\{j, j+1\}}^{-}\left(i q_{1}+\epsilon\right) \chi_{\{1,2\}}^{-}\left(-i q_{1}-\epsilon\right) \times\right. \\
& \times \int_{-\infty}^{\infty} \frac{d q_{2}}{2 \pi} \Omega_{\{j, j+1\}}^{-}\left(i q_{2}+\alpha\right) \Omega_{\{j, j+1\}}^{-}\left(-i q_{2}-\alpha\right)+ \\
& +\int_{-\infty}^{\infty} \frac{d q_{1}}{2 \pi}\left(i q_{1}+\epsilon\right) \Lambda_{\{1, j\}}\left(i q_{1}+\epsilon\right) \Omega_{\{j, j+1\}}^{-}\left(i q_{1}+\epsilon\right) \Omega_{\{1,2\}}^{-}\left(-i q_{1}-\epsilon\right) \times \\
& \left.\times \int_{-\infty}^{\infty} \frac{d q_{2}}{2 \pi} \chi_{\{j, j+1\}}^{-}\left(i q_{2}+\alpha\right) \chi_{\{j, j+1\}}^{-}\left(-i q_{2}-\alpha\right)\right)+ \\
& +T_{N}^{2} \int_{-\infty}^{\infty} \frac{d q_{1}}{2 \pi}\left(i q_{1}+\epsilon\right) \Lambda_{\{1, j\}}\left(i q_{1}+\epsilon\right) \Omega_{\{j, j+1\}}^{-}\left(i q_{1}+\epsilon\right) \Omega_{\{1,2\}}^{-}\left(-i q_{1}-\epsilon\right) \times \\
& \left.\times \int_{-\infty}^{\infty} \frac{d q_{2}}{2 \pi} \Omega_{\{j, j+1\}}^{-}\left(i q_{2}+\alpha\right) \Omega_{\{j, j+1\}}^{-}\left(-i q_{2}-\alpha\right)\right]
\end{aligned}
$$




$$
\begin{aligned}
& +\sum_{j=2}^{N}\left[T_{1}^{2} \int_{-\infty}^{\infty} \frac{d q_{1}}{2 \pi}\left(i q_{1}+\epsilon\right) \Gamma_{\{1, j\}}\left(-i q_{1}-\epsilon\right) \chi_{\{j-1, j\}}^{-}\left(-i q_{1}-\epsilon\right) \chi_{\{1,2\}}^{+}\left(i q_{1}+\epsilon\right) \times\right. \\
& \times \int_{-\infty}^{\infty} \frac{d q_{2}}{2 \pi} \chi_{\{j-1, j\}}^{-}\left(i q_{2}+\alpha\right) \chi_{\{j-1, j\}}^{-}\left(-i q_{2}-\alpha\right)+ \\
& +T_{1} T_{N}\left(\int_{-\infty}^{\infty} \frac{d q_{1}}{2 \pi}\left(i q_{1}+\epsilon\right) \Gamma_{\{1, j\}}\left(-i q_{1}-\epsilon\right) \chi_{\{j-1, j\}}^{-}\left(-i q_{1}-\epsilon\right) \chi_{\{1,2\}}^{+}\left(i q_{1}+\epsilon\right) \times\right. \\
& \times \int_{-\infty}^{\infty} \frac{d q_{2}}{2 \pi} \Omega_{\{j, j-1\}}^{-}\left(i q_{2}+\alpha\right) \Omega_{\{j-1, j\}}^{-}\left(-i q_{2}-\alpha\right)+ \\
& +\int_{-\infty}^{\infty} \frac{d q_{1}}{2 \pi}\left(i q_{1}+\epsilon\right) \Gamma_{\{1, j\}}\left(-i q_{1}-\epsilon\right) \Omega_{\{j-1, j\}}^{-}\left(-i q_{1}-\epsilon\right) \Omega_{\{1,2\}}^{+}\left(i q_{1}+\epsilon\right) \times \\
& \left.\times \int_{-\infty}^{\infty} \frac{d q_{2}}{2 \pi} \chi_{\{j-1, j\}}^{-}\left(i q_{2}+\alpha\right) \chi_{\{j-1, j\}}^{-}\left(-i q_{2}-\alpha\right)\right)+ \\
& +T_{N}^{2} \int_{-\infty}^{\infty} \frac{d q_{1}}{2 \pi}\left(i q_{1}+\epsilon\right) \Gamma_{\{1, j\}}\left(-i q_{1}-\epsilon\right) \Omega_{\{j-1, j\}}^{-}\left(-i q_{1}-\epsilon\right) \Omega_{\{1,2\}}^{+}\left(i q_{1}+\epsilon\right) \times \\
& \left.\times \int_{-\infty}^{\infty} \frac{d q_{2}}{2 \pi} \Omega_{\{j-1, j\}}^{-}\left(i q_{2}+\alpha\right) \Omega_{\{j-1, j\}}^{-}\left(-i q_{2}-\alpha\right)\right]+ \\
& -\sum_{j=1}^{N-1}\left[T_{1}^{2} \int_{-\infty}^{\infty} \frac{d q_{1}}{2 \pi}\left(i q_{1}+\epsilon\right) \Gamma_{\{1, j\}}\left(-i q_{1}-\epsilon\right) \chi_{\{j, j+1\}}^{-}\left(-i q_{1}-\epsilon\right) \chi_{\{1,2\}}^{+}\left(i q_{1}+\epsilon\right) \times\right. \\
& \times \int_{-\infty}^{\infty} \frac{d q_{2}}{2 \pi} \chi_{\{j, j+1\}}^{-}\left(i q_{2}+\alpha\right) \chi_{\{j, j+1\}}^{-}\left(-i q_{2}-\alpha\right)+ \\
& +T_{1} T_{N}\left(\int_{-\infty}^{\infty} \frac{d q_{1}}{2 \pi}\left(i q_{1}+\epsilon\right) \Gamma_{\{1, j\}}\left(-i q_{1}-\epsilon\right) \chi_{\{j, j+1\}}^{-}\left(-i q_{1}-\epsilon\right) \chi_{\{1,2\}}^{+}\left(i q_{1}+\epsilon\right) \times\right. \\
& \times \int_{-\infty}^{\infty} \frac{d q_{2}}{2 \pi} \Omega_{\{j, j+1\}}^{-}\left(i q_{2}+\alpha\right) \Omega_{\{j, j+1\}}^{-}\left(-i q_{2}-\alpha\right)+ \\
& +\int_{-\infty}^{\infty} \frac{d q_{1}}{2 \pi}\left(i q_{1}+\epsilon\right) \Gamma_{\{1, j\}}\left(-i q_{1}-\epsilon\right) \Omega_{\{j, j+1\}}^{-}\left(-i q_{1}-\epsilon\right) \Omega_{\{1,2\}}^{+}\left(i q_{1}+\epsilon\right) \times \\
& \left.\times \int_{-\infty}^{\infty} \frac{d q_{2}}{2 \pi} \chi_{\{j, j+1\}}^{-}\left(i q_{2}+\alpha\right) \chi_{\{j, j+1\}}^{-}\left(-i q_{2}-\alpha\right)\right)+ \\
& +T_{N}^{2} \int_{-\infty}^{\infty} \frac{d q_{1}}{2 \pi}\left(i q_{1}+\epsilon\right) \Gamma_{\{1, j\}}\left(-i q_{1}-\epsilon\right) \Omega_{\{j, j+1\}}^{-}\left(-i q_{1}-\epsilon\right) \Omega_{\{1,2\}}^{+}\left(i q_{1}+\epsilon\right) \times \\
& \left.\left.\times \int_{-\infty}^{\infty} \frac{d q_{2}}{2 \pi} \Omega_{\{j, j+1\}}^{-}\left(i q_{2}+\alpha\right) \Omega_{\{j, j+1\}}^{-}\left(-i q_{2}-\alpha\right)\right]\right\} .
\end{aligned}
$$

O fluxo de calor que atravessa o sistema nada mais é do que $I I+I$, onde esses termos são dados pelas equações (E-16) e (E-28), respectivamente. A forma condensada apresentada no texto através da Eq. (4-7), faz uso das seguintes definições para as funções que surgem no integrando do fluxo de calor: 


$$
\begin{aligned}
& \mathcal{I}_{1}=\int_{-\infty}^{\infty} \frac{d q_{1}}{2 \pi}\left(i q_{1}+\varepsilon\right) \chi_{\{1,2\}}^{+}\left(i q_{1}+\varepsilon\right) \chi_{\{1,2\}}^{-}\left(-i q_{1}-\varepsilon\right) \\
& \mathcal{I}_{2}=\int_{-\infty}^{\infty} \frac{d q_{1}}{2 \pi}\left(i q_{1}+\varepsilon\right) \Omega_{\{1,2\}}^{+}\left(i q_{1}+\varepsilon\right) \Omega_{\{1,2\}}^{-}\left(-i q_{1}-\varepsilon\right),
\end{aligned}
$$

onde estas funções são utilizadas apenas no termo $I I$. O restante das funções utilizadas no cálculo dos termos $I$ e $I I$, pode ser escrito da seguinte forma:

$$
\begin{aligned}
& \mathcal{I}_{3}^{[l, j]}=\int_{-\infty}^{\infty} \frac{d q_{1}}{2 \pi}\left(i q_{1}+\varepsilon\right) \Lambda_{l}\left(i q_{1}+\varepsilon\right) \chi_{\{j, j+1\}}^{-}\left(i q_{1}+\varepsilon\right) \chi_{\{1,2\}}^{-}\left(-i q_{1}-\varepsilon\right) \\
& \mathcal{I}_{4}^{[j]}=\int_{-\infty}^{\infty} \frac{d q_{2}}{2 \pi} \chi_{\{j, j+1\}}^{-}\left(i q_{2}+\alpha\right) \chi_{\{j, j+1\}}^{-}\left(-i q_{2}-\alpha\right) \\
& \mathcal{I}_{5}^{[l, j]}=\int_{-\infty}^{\infty} \frac{d q_{1}}{2 \pi}\left(i q_{1}+\varepsilon\right) \Lambda_{l}\left(i q_{1}+\varepsilon\right) \Omega_{\{j, j+1\}}^{-}\left(i q_{1}+\varepsilon\right) \Omega_{\{1,2\}}^{-}\left(-i q_{1}-\varepsilon\right) \\
& \mathcal{I}_{6}^{[j]}=\int_{-\infty}^{\infty} \frac{d q_{2}}{2 \pi} \Omega_{\{j, j+1\}}^{-}\left(i q_{2}+\alpha\right) \Omega_{\{j, j+1\}}^{-}\left(-i q_{2}-\alpha\right) \\
& \mathcal{I}_{7}^{[l, j]}=\int_{-\infty}^{\infty} \frac{d q_{1}}{2 \pi}\left(i q_{1}+\varepsilon\right) \Gamma_{l}\left(-i q_{1}-\varepsilon\right) \chi_{\{j, j+1\}}^{-}\left(-i q_{1}-\varepsilon\right) \chi_{\{1,2\}}^{+}\left(i q_{1}+\varepsilon\right) \\
& \mathcal{I}_{8}^{[l, j]}=\int_{-\infty}^{\infty} \frac{d q_{1}}{2 \pi}\left(i q_{1}+\varepsilon\right) \Gamma_{l}\left(-i q_{1}-\varepsilon\right) \Omega_{\{j, j+1\}}^{-}\left(-i q_{1}-\varepsilon\right) \Omega_{\{1,2\}}^{+}\left(i q_{1}+\varepsilon\right),
\end{aligned}
$$

representando assim todas as funções necessárias para calcular o fluxo de calor quando o sistema encontra-se sujeito a reservatórios gaussianos. Para computar o fluxo de calor para uma cadeia alimentada por ruídos de Poisson, basta introduzir as funções decorrentes dos termos de médias quárticas presentes em $I$ e $I I$, de forma que estas são definidas como: 


$$
\begin{aligned}
& \mathcal{I}_{9}^{[j]}=\int_{\text {all space }} \frac{d q_{1}}{2 \pi} \frac{d q_{2}}{2 \pi} \frac{d q_{3}}{2 \pi} \chi_{\{j, j+1\}}^{-}\left(i q_{1}+\varepsilon\right) \chi_{\{j, j+1\}}^{-}\left(i q_{2}+\varepsilon\right) \times \\
& \times \chi_{\{j, j+1\}}^{-}\left(-i q_{1}-i q_{2}-i q_{3}-3 \varepsilon\right) \chi_{\{1,2\}}^{+}\left(i q_{3}+\varepsilon\right) \\
& \mathcal{I}_{10}^{[j]}=\int_{\text {all space }} \frac{d q_{1}}{2 \pi} \frac{d q_{2}}{2 \pi} \frac{d q_{3}}{2 \pi} \Omega_{\{j, j+1\}}^{-}\left(i q_{1}+\varepsilon\right) \Omega_{\{j, j+1\}}^{-}\left(i q_{2}+\varepsilon\right) \times \\
& \times \Omega_{\{j, j+1\}}^{-}\left(-i q_{1}-i q_{2}-i q_{3}-3 \varepsilon\right) \Omega_{\{1,2\}}^{+}\left(i q_{3}+\varepsilon\right) \\
& \mathcal{I}_{11}^{[l, j]}=-\int_{\text {all space }} \frac{d q_{1}}{2 \pi} \frac{d q_{2}}{2 \pi} \frac{d q_{3}}{2 \pi}\left(i q_{1}+\varepsilon\right) \chi_{\{1,2\}}^{-}\left(i q_{1}+\varepsilon\right) \Lambda_{l}\left(-i q_{1}-\varepsilon\right) \chi_{\{j, j+1\}}^{-}\left(i q_{2}+\varepsilon\right) \times
\end{aligned}
$$

$$
\begin{aligned}
& \times \chi_{\{j, j+1\}}^{-}\left(i q_{3}+\varepsilon\right) \chi_{\{j, j+1\}}^{-}\left(-i q_{1}-i q_{2}-i q_{3}-3 \varepsilon\right) \\
& \mathcal{I}_{12}^{[l, j]}=-\int_{\text {all space }} \frac{d q_{1}}{2 \pi} \frac{d q_{2}}{2 \pi} \frac{d q_{3}}{2 \pi}\left(i q_{1}+\varepsilon\right) \Omega_{\{1,2\}}^{-}\left(i q_{1}+\varepsilon\right) \Lambda_{l}\left(-i q_{1}-\varepsilon\right) \Omega_{\{j, j+1\}}^{-}\left(i q_{2}+\varepsilon\right) \times
\end{aligned}
$$

$$
\begin{aligned}
& \times \Omega_{\{j, j+1\}}^{-}\left(i q_{3}+\varepsilon\right) \Omega_{\{j, j+1\}}^{-}\left(-i q_{1}-i q_{2}-i q_{3}-3 \varepsilon\right) \\
& \mathcal{I}_{13}^{[l, j]}=\int_{\text {all space }} \frac{d q_{1}}{2 \pi} \frac{d q_{2}}{2 \pi} \frac{d q_{3}}{2 \pi}\left(i q_{1}+\varepsilon\right) \chi_{\{1,2\}}^{+}\left(i q_{1}+\varepsilon\right) \Gamma_{l}\left(-i q_{1}-\varepsilon\right) \chi_{\{j, j+1\}}^{-}\left(i q_{2}+\varepsilon\right) \times
\end{aligned}
$$

$$
\begin{aligned}
& \times \chi_{\{j, j+1\}}^{-}\left(i q_{3}+\varepsilon\right) \chi_{\{j, j+1\}}^{-}\left(-i q_{1}-i q_{2}-i q_{3}-3 \varepsilon\right) \\
& \mathcal{I}_{14}^{[l, j]}=\int_{\text {all space }} \frac{d q_{1}}{2 \pi} \frac{d q_{2}}{2 \pi} \frac{d q_{3}}{2 \pi}\left(i q_{1}+\varepsilon\right) \Omega_{\{1,2\}}^{+}\left(i q_{1}+\varepsilon\right) \Gamma_{l}\left(-i q_{1}-\varepsilon\right) \Omega_{\{j, j+1\}}^{-}\left(i q_{2}+\varepsilon\right) \times
\end{aligned}
$$

$$
\times \Omega_{\{j, j+1\}}^{-}\left(i q_{3}+\varepsilon\right) \Omega_{\{j, j+1\}}^{-}\left(-i q_{1}-i q_{2}-i q_{3}-3 \varepsilon\right)
$$

\section{E.3}

\section{Temperatura das partículas da cadeia}

Utilizando conceitos simples de termodinâmica, tem-se que a temperatura de uma partícula é definida como:

$$
\mathcal{T}_{i} \equiv m\left\langle v_{i}^{2}\right\rangle .
$$


Usando a transformada de Fourier-Laplace em (E-44), tem-se:

$$
\begin{aligned}
\mathcal{T}_{i}= & m \lim _{z \rightarrow 0, \epsilon \rightarrow 0} \int \frac{d q_{1}}{2 \pi} \frac{d q_{2}}{2 \pi} \frac{z}{z-\left(\mathrm{i} q_{1}+\mathrm{i} q_{2}+2 \epsilon\right)} \times \\
& \times\left\langle\tilde{v}_{i}\left(\mathrm{i} q_{1}+\epsilon\right) \tilde{v}_{i}\left(\mathrm{i} q_{2}+\epsilon\right)\right\rangle= \\
& =m \lim _{z \rightarrow 0, \epsilon \rightarrow 0} \int \frac{d q_{1}}{2 \pi} \frac{d q_{2}}{2 \pi} \frac{z\left(\mathrm{i} q_{1}+\epsilon\right)\left(\mathrm{i} q_{2}+\epsilon\right)}{z-\left(\mathrm{i} q_{1}+\mathrm{i} q_{2}+2 \epsilon\right)} \times \\
& \times\left\langle\tilde{x}_{i}\left(\mathrm{i} q_{1}+\epsilon\right) \tilde{x}_{i}\left(\mathrm{i} q_{2}+\varepsilon\right)\right\rangle .
\end{aligned}
$$

Assim como foi feito na análise do fluxo de calor, foca-se apenas na expansão do núcleo do numerador do integrando de interesse, neste caso dado por (E-45). Utilizando (E-9), tem-se que (E-45) é reescrita como

$$
\left[\left\langle\widetilde{\mathbf{x}}_{i}^{(0)}\left(i q_{1}+\epsilon\right) \widetilde{\mathbf{x}}_{i}^{(0)}\left(i q_{2}+\epsilon\right)\right\rangle+2 \delta\left\langle\widetilde{\mathbf{x}}_{i}^{(0)}\left(i q_{1}+\epsilon\right) \widetilde{\mathbf{x}}_{i}^{(1)}\left(i q_{2}+\epsilon\right)\right\rangle\right]\left(i q_{1}+\epsilon\right)\left(i q_{2}+\epsilon\right)
$$

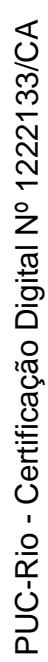

A primeira contribuição de (E-46) está associada ao perfil um tanto estranho obtido no caso linear, de forma que toda nova informação em termos da distribuição de temperaturas do sistema advém da segunda contribuição, que 
quando expandida com a ajuda de (E-18), (E-20), (E-21) e (E-22) é dada por:

$$
\begin{aligned}
& 6(2 \gamma)^{2} k_{3}\left\{\sum _ { j = 1 } ^ { N - 1 } \left[T_{1}^{2} \int_{-\infty}^{\infty} \frac{d q_{1}}{2 \pi}\left(i q_{1}+\epsilon\right)^{2} \tilde{\mathcal{A}}_{\{i, 1\}}\left(i q_{1}+\epsilon\right) \tilde{\mathcal{A}}_{\{i, j\}}\left(-i q_{1}-\epsilon\right) \chi_{\{j, j+1\}}^{-}\left(-i q_{1}-\epsilon\right) \times\right.\right. \\
& \times \int_{-\infty}^{\infty} \frac{d q_{2}}{2 \pi} \chi_{\{j, j+1\}}^{-}\left(i q_{2}+\epsilon\right) \chi_{\{j, j+1\}}^{-}\left(-i q_{2}-\epsilon\right)+ \\
& +T_{1} T_{N} \int_{-\infty}^{\infty} \frac{d q_{1}}{2 \pi}\left(i q_{1}+\epsilon\right)^{2} \tilde{\mathcal{A}}_{\{i, 1\}}\left(i q_{1}+\epsilon\right) \tilde{\mathcal{A}}_{\{i, j\}}\left(-i q_{1}-\epsilon\right) \chi_{\{j, j+1\}}^{-}\left(-i q_{1}-\epsilon\right) \times \\
& \times \int_{-\infty}^{\infty} \frac{d q_{2}}{2 \pi} \Omega_{\{j, j+1\}}^{-}\left(i q_{2}+\epsilon\right) \Omega_{\{j, j+1\}}^{-}\left(-i q_{2}-\epsilon\right)+ \\
& +T_{1} T_{N} \int_{-\infty}^{\infty} \frac{d q_{1}}{2 \pi}\left(i q_{1}+\epsilon\right)^{2} \tilde{\mathcal{A}}_{\{i, N\}}\left(i q_{1}+\epsilon\right) \tilde{\mathcal{A}}_{\{i, j\}}\left(-i q_{1}-\epsilon\right) \Omega_{\{j, j+1\}}^{-}\left(-i q_{1}-\epsilon\right) \times \\
& \times \int_{-\infty}^{\infty} \frac{d q_{2}}{2 \pi} \chi_{\{j, j+1\}}^{-}\left(i q_{2}+\epsilon\right) \chi_{\{j, j+1\}}^{-}\left(-i q_{2}-\epsilon\right)+ \\
& +T_{N}^{2} \int_{-\infty}^{\infty} \frac{d q_{1}}{2 \pi}\left(i q_{1}+\epsilon\right)^{2} \widetilde{\mathcal{A}}_{\{i, N\}}\left(i q_{1}+\epsilon\right) \widetilde{\mathcal{A}}_{\{i, 1\}}\left(-i q_{1}-\epsilon\right) \Omega_{\{j, j+1\}}^{-}\left(-i q_{1}-\epsilon\right) \times \\
& \left.\times \int_{-\infty}^{\infty} \frac{d q_{2}}{2 \pi} \Omega_{\{j, j+1\}}^{-}\left(i q_{2}+\epsilon\right) \Omega_{\{j, j+1\}}^{-}\left(-i q_{2}-\epsilon\right)\right]+ \\
& -\sum_{j=2}^{N}\left[T_{1}^{2} \int_{-\infty}^{\infty} \frac{d q_{1}}{2 \pi}\left(i q_{1}+\epsilon\right)^{2} \widetilde{\mathcal{A}}_{\{i, 1\}}\left(i q_{1}+\epsilon\right) \widetilde{\mathcal{A}}_{\{i, j\}}\left(-i q_{1}-\epsilon\right) \chi_{\{j-1, j\}}^{-}\left(-i q_{1}-\epsilon\right) \times\right. \\
& \times \int_{-\infty}^{\infty} \frac{d q_{2}}{2 \pi} \chi_{\{j, j+1\}}^{-}\left(i q_{2}+\epsilon\right) \chi_{\{j-1, j\}}^{-}\left(-i q_{2}-\epsilon\right)+ \\
& +T_{1} T_{N} \int_{-\infty}^{\infty} \frac{d q_{1}}{2 \pi}\left(i q_{1}+\epsilon\right)^{2} \widetilde{\mathcal{A}}_{\{i, 1\}}\left(i q_{1}+\epsilon\right) \widetilde{\mathcal{A}}_{\{i, j\}}\left(-i q_{1}-\epsilon\right) \chi_{\{j-1, j\}}^{-}\left(-i q_{1}-\epsilon\right) \times \\
& \times \int_{-\infty}^{\infty} \frac{d q_{2}}{2 \pi} \Omega_{\{j, j+1\}}^{-}\left(i q_{2}+\epsilon\right) \Omega_{\{j-1, j\}}^{-}\left(-i q_{2}-\epsilon\right)+ \\
& +T_{1} T_{N} \int_{-\infty}^{\infty} \frac{d q_{1}}{2 \pi}\left(i q_{1}+\epsilon\right)^{2} \widetilde{\mathcal{A}}_{\{i, N\}}\left(i q_{1}+\epsilon\right) \tilde{\mathcal{A}}_{\{i, j\}}\left(-i q_{1}-\epsilon\right) \Omega_{\{j-1, j\}}^{-}\left(-i q_{1}-\epsilon\right) \times \\
& \times \int_{-\infty}^{\infty} \frac{d q_{2}}{2 \pi} \chi_{\{j, j+1\}}^{-}\left(i q_{2}+\epsilon\right) \chi_{\{j-1, j\}}^{-}\left(-i q_{2}-\epsilon\right)+ \\
& +T_{N}^{2} \int_{-\infty}^{\infty} \frac{d q_{1}}{2 \pi}\left(i q_{1}+\epsilon\right)^{2} \widetilde{\mathcal{A}}_{\{i, N\}}\left(i q_{1}+\epsilon\right) \tilde{\mathcal{A}}_{\{i, j\}}\left(-i q_{1}-\epsilon\right) \Omega_{\{j-1, j\}}^{-}\left(-i q_{1}-\epsilon\right) \times \\
& \left.\left.\times \int_{-\infty}^{\infty} \frac{d q_{2}}{2 \pi} \Omega_{\{j, j+1\}}^{-}\left(i q_{2}+\epsilon\right) \Omega_{\{j-1, j\}}^{-}\left(-i q_{2}-\epsilon\right)\right]\right\}
\end{aligned}
$$


Finalmente, a temperatura de cada sítio é escrita como

$$
\begin{aligned}
& \mathcal{T}_{i}=\frac{m \gamma}{\pi} \sum_{j=1, N} T_{j} \int_{-\infty}^{\infty} d q q^{2} \tilde{\mathcal{A}}_{i j}(\mathrm{i} q+\varepsilon) \tilde{\mathcal{A}}_{i j}(-\mathrm{i} q-\varepsilon)+ \\
& +6 m(2 \gamma)^{2} k_{3}\left\{\sum _ { j = 1 } ^ { N - 1 } \left[T_{1}^{2} \int_{-\infty}^{\infty} \frac{d q_{1}}{2 \pi}\left(i q_{1}+\epsilon\right)^{2} \widetilde{\mathcal{A}}_{\{i, 1\}}\left(i q_{1}+\epsilon\right) \tilde{\mathcal{A}}_{\{i, j\}}\left(-i q_{1}-\epsilon\right) \chi_{\{j, j+1\}}^{-}\left(-i q_{1}-\epsilon\right) \times\right.\right. \\
& \times \int_{-\infty}^{\infty} \frac{d q_{2}}{2 \pi} \chi_{\{j, j+1\}}^{-}\left(i q_{2}+\epsilon\right) \chi_{\{j, j+1\}}^{-}\left(-i q_{2}-\epsilon\right)+ \\
& +T_{1} T_{N} \int_{-\infty}^{\infty} \frac{d q_{1}}{2 \pi}\left(i q_{1}+\epsilon\right)^{2} \widetilde{\mathcal{A}}_{\{i, 1\}}\left(i q_{1}+\epsilon\right) \widetilde{\mathcal{A}}_{\{i, j\}}\left(-i q_{1}-\epsilon\right) \chi_{\{j, j+1\}}^{-}\left(-i q_{1}-\epsilon\right) \times \\
& \times \int_{-\infty}^{\infty} \frac{d q_{2}}{2 \pi} \Omega_{\{j, j+1\}}^{-}\left(i q_{2}+\epsilon\right) \Omega_{\{j, j+1\}}^{-}\left(-i q_{2}-\epsilon\right)+ \\
& +T_{1} T_{N} \int_{-\infty}^{\infty} \frac{d q_{1}}{2 \pi}\left(i q_{1}+\epsilon\right)^{2} \widetilde{\mathcal{A}}_{\{i, N\}}\left(i q_{1}+\epsilon\right) \widetilde{\mathcal{A}}_{\{i, j\}}\left(-i q_{1}-\epsilon\right) \Omega_{\{j, j+1\}}^{-}\left(-i q_{1}-\epsilon\right) \times \\
& \times \int_{-\infty}^{\infty} \frac{d q_{2}}{2 \pi} \chi_{\{j, j+1\}}^{-}\left(i q_{2}+\epsilon\right) \chi_{\{j, j+1\}}^{-}\left(-i q_{2}-\epsilon\right)+ \\
& +T_{N}^{2} \int_{-\infty}^{\infty} \frac{d q_{1}}{2 \pi}\left(i q_{1}+\epsilon\right)^{2} \widetilde{\mathcal{A}}_{\{i, N\}}\left(i q_{1}+\epsilon\right) \widetilde{\mathcal{A}}_{\{i, j\}}\left(-i q_{1}-\epsilon\right) \Omega_{\{j, j+1\}}^{-}\left(-i q_{1}-\epsilon\right) \times \\
& \left.\times \int_{-\infty}^{\infty} \frac{d q_{2}}{2 \pi} \Omega_{\{j, j+1\}}^{-}\left(i q_{2}+\epsilon\right) \Omega_{\{j, j+1\}}^{-}\left(-i q_{2}-\epsilon\right)\right]+ \\
& -\sum_{j=2}^{N}\left[T_{1}^{2} \int_{-\infty}^{\infty} \frac{d q_{1}}{2 \pi}\left(i q_{1}+\epsilon\right)^{2} \tilde{\mathcal{A}}_{\{i, 1\}}\left(i q_{1}+\epsilon\right) \tilde{\mathcal{A}}_{\{i, j\}}\left(-i q_{1}-\epsilon\right) \chi_{\{j-1, j\}}^{-}\left(-i q_{1}-\epsilon\right) \times\right. \\
& \times \int_{-\infty}^{\infty} \frac{d q_{2}}{2 \pi} \chi_{\{j-1, j\}}^{-}\left(i q_{2}+\epsilon\right) \chi_{\{j-1, j\}}^{-}\left(-i q_{2}-\epsilon\right)+ \\
& +T_{1} T_{N} \int_{-\infty}^{\infty} \frac{d q_{1}}{2 \pi}\left(i q_{1}+\epsilon\right)^{2} \widetilde{\mathcal{A}}_{\{i, 1\}}\left(i q_{1}+\epsilon\right) \tilde{\mathcal{A}}_{\{i, j\}}\left(-i q_{1}-\epsilon\right) \chi_{\{j-1, j\}}^{-}\left(-i q_{1}-\epsilon\right) \times \\
& \times \int_{-\infty}^{\infty} \frac{d q_{2}}{2 \pi} \Omega_{\{j-1, j\}}^{-}\left(i q_{2}+\epsilon\right) \Omega_{\{j-1, j\}}^{-}\left(-i q_{2}-\epsilon\right)+ \\
& +T_{1} T_{N} \int_{-\infty}^{\infty} \frac{d q_{1}}{2 \pi}\left(i q_{1}+\epsilon\right)^{2} \widetilde{\mathcal{A}}_{\{i, N\}}\left(i q_{1}+\epsilon\right) \tilde{\mathcal{A}}_{\{i, j\}}\left(-i q_{1}-\epsilon\right) \Omega_{\{j-1, j\}}^{-}\left(-i q_{1}-\epsilon\right) \times \\
& \times \int_{-\infty}^{\infty} \frac{d q_{2}}{2 \pi} \chi_{\{j-1, j\}}^{-}\left(i q_{2}+\epsilon\right) \chi_{\{j-1, j\}}^{-}\left(-i q_{2}-\epsilon\right)+ \\
& +T_{N}^{2} \int_{-\infty}^{\infty} \frac{d q_{1}}{2 \pi}\left(i q_{1}+\epsilon\right)^{2} \widetilde{\mathcal{A}}_{\{i, N\}}\left(i q_{1}+\epsilon\right) \tilde{\mathcal{A}}_{\{i, j\}}\left(-i q_{1}-\epsilon\right) \Omega_{\{j-1, j\}}^{-}\left(-i q_{1}-\epsilon\right) \times \\
& \left.\left.\times \int_{-\infty}^{\infty} \frac{d q_{2}}{2 \pi} \Omega_{\{j-1, j\}}^{-}\left(i q_{2}+\epsilon\right) \Omega_{\{j-1, j\}}^{-}\left(-i q_{2}-\epsilon\right)\right]\right\}
\end{aligned}
$$


$\mathbf{F}$

\section{Resultados suplementares dos cumulantes da cadeia harmônica linear}

Ao longo do capítulo 3 mostrou-se alguns resultados sobre os cumulantes da cadeia harmônica linear, mas o passo a passo analítico não foi detalhado. Neste apêndice apresenta-se explicitamente o desenvolvimento de $\left\langle J_{j \rightarrow j+1}^{2}\right\rangle$ para que o(a) leitor(a) entenda o procedimento utilizado nos cálculos dos demais cumulantes, que são bem semelhantes,salvo pelo trabalho exigido por cada um deles. Olhando para a equação 4-6 e utilizando $n=2$, tem-se que no espaço de Fourier-Laplace a média quadrática do fluxo de calor entre partículas vizinhas é,

$$
\begin{aligned}
& \left\langle J_{j \rightarrow j+1}^{2}\right\rangle=\frac{k_{1}^{2}}{4} \lim _{z, \epsilon \rightarrow 0} \int_{-\infty}^{\infty} \int_{-\infty}^{\infty} \int_{-\infty}^{\infty} \int_{-\infty}^{\infty} \frac{d q_{1}}{2 \pi} \frac{d q_{2}}{2 \pi} \frac{d q_{3}}{2 \pi} \frac{d q_{4}}{2 \pi} \frac{z}{z-\left(i q_{1}+i q_{2}+i q_{3}+i q_{4}+4 \epsilon\right)} \times \\
& \times\left\langle\left[\tilde{x}_{j}\left(i q_{1}+\epsilon\right) \tilde{x}_{j}\left(i q_{2}+\epsilon\right)+\tilde{x}_{j}\left(i q_{1}+\epsilon\right) \tilde{x}_{j+1}\left(i q_{2}+\epsilon\right)-\tilde{x}_{j}\left(i q_{2}+\epsilon\right) \tilde{x}_{j+1}\left(i q_{1}+\epsilon\right)+\right.\right. \\
& \left.-\tilde{x}_{j+1}\left(i q_{1}+\epsilon\right) \tilde{x}_{j+1}\left(i q_{2}+\epsilon\right)\right]\left[\tilde{x}_{j}\left(i q_{3}+\epsilon\right) \tilde{x}_{j}\left(i q_{4}+\epsilon\right)+\tilde{x}_{j}\left(i q_{3}+\epsilon\right) \tilde{x}_{j+1}\left(i q_{4}+\epsilon\right)+\right. \\
& \left.\left.-\tilde{x}_{j}\left(i q_{4}+\epsilon\right) \tilde{x}_{j+1}\left(i q_{3}+\epsilon\right)-\tilde{x}_{j+1}\left(i q_{3}+\epsilon\right) \tilde{x}_{j+1}\left(i q_{4}+\epsilon\right)\right]\right\rangle\left(i q_{2}+\epsilon\right)\left(i q_{4}+\epsilon\right) .
\end{aligned}
$$

Usando (2-14) e (E-11) pode se reescrever a expressão acima como:

$$
\begin{aligned}
& \left\langle J_{j \rightarrow j+1}^{2}\right\rangle=\frac{k_{1}^{2}}{4} \lim _{z, \epsilon \rightarrow 0} \int_{-\infty}^{\infty} \int_{-\infty}^{\infty} \int_{-\infty}^{\infty} \int_{-\infty}^{\infty} \frac{d q_{1}}{2 \pi} \frac{d q_{2}}{2 \pi} \frac{d q_{3}}{2 \pi} \frac{d q_{4}}{2 \pi} \frac{z}{z-\left(i q_{1}+i q_{2}+i q_{3}+i q_{4}+4 \epsilon\right)} \times \\
& \times\left(i q_{2}+\epsilon\right)\left(i q_{4}+\epsilon\right)\left\langle\left[\omega^{-}\left(i q_{1}+\epsilon\right) \omega^{+}\left(i q_{2}+\epsilon\right) \omega^{-}\left(i q_{3}+\epsilon\right) \omega^{+}\left(i q_{4}+\epsilon\right)+\right.\right. \\
& +4 \omega^{-}\left(i q_{1}+\epsilon\right) \omega^{+}\left(i q_{2}+\epsilon\right) \phi^{-}\left(i q_{3}+\epsilon\right) \phi^{+}\left(i q_{4}+\epsilon\right)+ \\
& \omega^{-}\left(i q_{1}+\epsilon\right) \phi^{+}\left(i q_{2}+\epsilon\right) \omega^{-}+\left(i q_{3}+\epsilon\right) \phi^{+}\left(i q_{4}+\epsilon\right)+ \\
& +\phi^{-}\left(i q_{1}+\epsilon\right) \omega^{+}\left(i q_{2}+\epsilon\right) \phi^{-}\left(i q_{3}+\epsilon\right) \omega^{+}\left(i q_{4}+\epsilon\right)+ \\
& \left.\left.+\phi^{-}\left(i q_{1}+\epsilon\right) \phi^{+}\left(i q_{2}+\epsilon\right) \phi^{-}\left(i q_{3}+\epsilon\right) \phi^{+}\left(i q_{4}+\epsilon\right)\right]\right\rangle
\end{aligned}
$$

Nota-se que para resolver estas integrais é necessário calcular médias do tipo, $\left\langle\tilde{\eta}_{i}\left(i q_{\alpha}+\epsilon\right) \tilde{\eta}_{j}\left(i q_{\beta}+\epsilon\right) \tilde{\eta}_{k}\left(i q_{\lambda}+\epsilon\right) \tilde{\eta}_{l}\left(i q_{\theta}+\epsilon\right)\right\rangle,(i, j, k, l) \in\{1, N\}$ e $(\alpha, \beta, \lambda, \theta) \in$ $\{1,2,3,4\}$. Pode se escrever estas médias de uma maneira mais tratável, explorando o fato de que o ruído empregado no problema é do tipo gaussiano branco. O teorema de Wick para neste caso pode ser escrito como,

$$
\begin{gathered}
\left\langle\tilde{\eta}_{i 1} \tilde{\eta}_{i 2} \tilde{\eta}_{i 3} \tilde{\eta}_{i 4}\right\rangle=\left\langle\tilde{\eta}_{i 1} \tilde{\eta}_{i 2}\right\rangle_{c}\left\langle\tilde{\eta}_{i 3} \tilde{\eta}_{i 4}\right\rangle_{c}+\left\langle\tilde{\eta}_{i 1} \tilde{\eta}_{i 3}\right\rangle_{c}\left\langle\tilde{\eta}_{i 2} \tilde{\eta}_{i 4}\right\rangle_{c}+\left\langle\tilde{\eta}_{i 1} \tilde{\eta}_{i 4}\right\rangle_{c}\left\langle\tilde{\eta}_{i 2} \tilde{\eta}_{i 3}\right\rangle_{c} \\
\left\langle\tilde{\eta}_{1 i} \tilde{\eta}_{1 j} \tilde{\eta}_{2 l} \tilde{\eta}_{2 m}\right\rangle=\left\langle\tilde{\eta}_{1 i} \tilde{\eta}_{1 j}\right\rangle_{c}\left\langle\tilde{\eta}_{2 l} \tilde{\eta}_{2 m}\right\rangle_{c}
\end{gathered}
$$

Substituindo as Eqs. (F-3) e (F-4) na (F-2), temos que a média quadrática do fluxo de calor é, 


$$
\begin{aligned}
& \left\langle J^{2}\right\rangle_{j \rightarrow j+1}= \\
& \lim _{\epsilon \rightarrow 0} \frac{k_{1}^{2}}{4}\left[4 \gamma ^ { 2 } T _ { N } ^ { 2 } \left(2 \int_{-\infty}^{\infty} \int_{-\infty}^{\infty} \frac{d q_{1}}{2 \pi} \frac{d q_{2}}{2 \pi}\left(i q_{1}+\epsilon\right)\left(i q_{2}+\epsilon\right) \Omega^{+}\left(i q_{1}+\epsilon\right) \Omega^{-}\left(-i q_{1}-\epsilon\right) \Omega^{+}\left(i q_{2}+\epsilon\right) \Omega^{-}\left(-i q_{2}-\epsilon\right)\right.\right. \\
& \left.+\int_{-\infty}^{\infty} \int_{-\infty}^{\infty} \frac{d q_{1}}{2 \pi} \frac{d q_{2}}{2 \pi}\left(i q_{2}+\epsilon\right)^{2} \Omega^{-}\left(i q_{1}+\epsilon\right) \Omega^{-}\left(-i q_{1}-\epsilon\right) \Omega^{+}\left(i q_{2}+\epsilon\right) \Omega^{+}\left(-i q_{2}-\epsilon\right)\right)+ \\
& +4 \gamma^{2} T_{1} T_{N}\left(4 \int_{-\infty}^{\infty} \int_{-\infty}^{\infty} \frac{d q_{1}}{2 \pi} \frac{d q_{2}}{2 \pi}\left(i q_{1}+\epsilon\right)\left(i q_{2}+\epsilon\right) \chi^{+}\left(i q_{1}+\epsilon\right) \chi^{-}\left(-i q_{1}-\epsilon\right) \Omega^{+}\left(i q_{2}+\epsilon\right) \Omega^{-}\left(-i q_{2}-\epsilon\right)+\right. \\
& \int_{-\infty}^{\infty} \int_{-\infty}^{\infty} \frac{d q_{1}}{2 \pi} \frac{d q_{2}}{2 \pi}\left(i q_{2}+\epsilon\right)^{2} \chi^{+}\left(i q_{2}+\epsilon\right) \chi^{+}\left(-i q_{2}-\epsilon\right) \Omega^{-}\left(i q_{1}+\epsilon\right) \Omega^{-}\left(-i q_{1}-\epsilon\right)+ \\
& \left.+\int_{-\infty}^{\infty} \int_{-\infty}^{\infty} \frac{d q_{1}}{2 \pi} \frac{d q_{2}}{2 \pi}\left(i q_{2}+\epsilon\right)^{2} \Omega^{+}\left(i q_{2}+\epsilon\right) \Omega^{+}\left(-i q_{2}-\epsilon\right) \chi^{-}\left(i q_{1}+\epsilon\right) \chi^{-}\left(-i q_{1}-\epsilon\right)\right)+ \\
& +4 \gamma^{2} T_{1}^{2}\left(2 \int_{-\infty}^{\infty} \int_{-\infty}^{\infty} \frac{d q_{1}}{2 \pi} \frac{d q_{2}}{2 \pi}\left(i q_{1}+\epsilon\right)\left(i q_{2}+\epsilon\right) \chi^{+}\left(i q_{1}+\epsilon\right) \chi^{-}\left(-i q_{1}-\epsilon\right) \chi^{+}\left(i q_{2}+\epsilon\right) \chi^{-}\left(-i q_{2}-\epsilon\right)\right. \\
& \left.\left.+\int_{-\infty}^{\infty} \int_{-\infty}^{\infty} \frac{d q_{1}}{2 \pi} \frac{d q_{2}}{2 \pi}\left(i q_{2}+\epsilon\right)^{2} \chi^{-}\left(i q_{1}+\epsilon\right) \chi^{-}\left(-i q_{1}-\epsilon\right) \chi^{+}\left(i q_{2}+\epsilon\right) \chi^{+}\left(-i q_{2}-\epsilon\right)\right)\right] .
\end{aligned}
$$

Como deseja-se encontrar o cumulante desta grandeza, deve calcular-se

$$
\left\langle J^{2}\right\rangle_{c}=\left\langle J^{2}\right\rangle-\langle J\rangle^{2}
$$

com o fluxo de calor dado por 2-22. A comparação do resultado analítico versus numérico do segundo cumulante do fluxo de calor pode ser visto nas figuras F.2(a) e F.1. O cálculo do terceiro e quarto cumulantes é bem similar ao feito para determinar a variância, exceto pela demanda de trabalho exigida. Como o ruído é gaussiano, a quantidade de termos a ser calculada é igual ao somatório do número de partições tomadas dois a dois possíveis, que é escrito como:

$$
\Sigma \# \text { Partições possíveis }=\frac{n !}{\left(\frac{n}{2}\right) ! 2^{\frac{n}{2}}},
$$

onde $n$ é a ordem da média a ser calculada. Nota-se que um termo do tipo $\left\langle\tilde{\eta}_{i 1} \tilde{\eta}_{i 2} \tilde{\eta}_{i 3} \tilde{\eta}_{i 4} \tilde{\eta}_{i 5} \tilde{\eta}_{i 6}\right\rangle$, que surge no cálculo da obliquidade possui 15 termos de produtos de partições dois a dois do ruído, enquanto uma média do tipo $\left\langle\tilde{\eta}_{i 1} \tilde{\eta}_{i 2} \tilde{\eta}_{i 3} \tilde{\eta}_{i 4} \tilde{\eta}_{i 5} \tilde{\eta}_{i 6} \tilde{\eta}_{i 7} \tilde{\eta}_{i 8}\right\rangle$ contém 105 destes termos! Por este motivo não mostra-se o desenvolvimento das expressões para a média cúbica e quártica do fluxo de calor ao longo do texto. Assim, as definições [22] do terceiro e quarto cumulantes utilizadas nos resultados mostrados são, respectivamente:

$$
\begin{gathered}
\left\langle J^{3}\right\rangle_{c}=\left\langle J^{3}\right\rangle-3\left\langle J^{2}\right\rangle\langle J\rangle+2\langle J\rangle^{3} \\
\left\langle J^{4}\right\rangle_{c}=\left\langle J^{4}\right\rangle-4\left\langle J^{3}\right\rangle\langle J\rangle-3\left\langle J^{2}\right\rangle^{2}+12\left\langle J^{2}\right\rangle\langle J\rangle^{2}-6\langle J\rangle^{4}
\end{gathered}
$$

De acordo com a figura F.1 nota-se que o fato de o sistema ser linear implica em uma saturação no valor dos demais cumulantes da distribuição, ainda que seja de forma mais lenta. Logo, em uma cadeia harmônica conclui-se que, a 
Apêndice F. Resultados suplementares dos cumulantes da cadeia harmônica linear
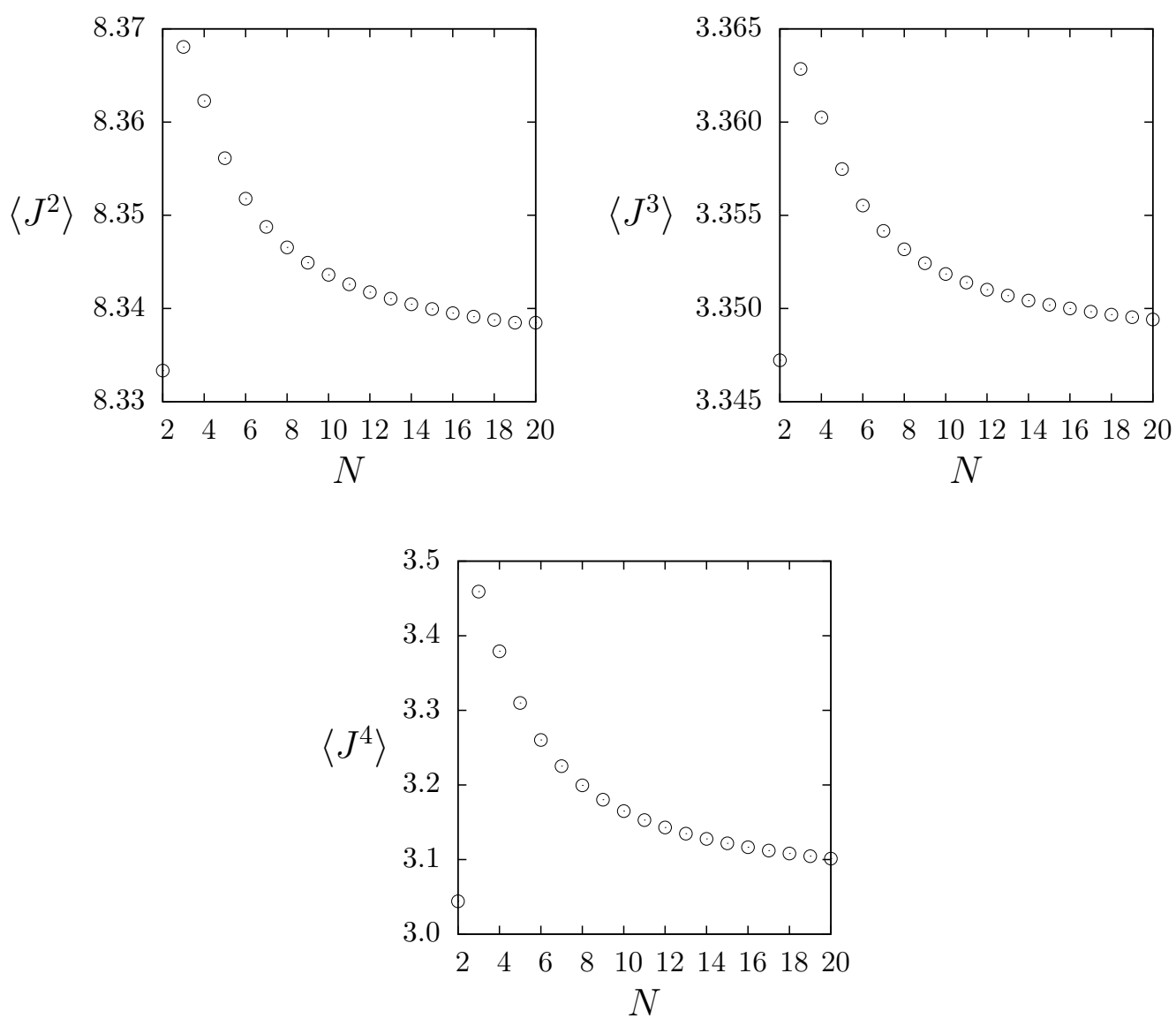

Figura F.1: Comportamento da variância, obliquidade e curtose do fluxo de calor $J$ em uma cadeia harmônica linear em função do número $N$ de elementos do sistema

partir de um tamanho finito da cadeia, o valor dos cumulantes da distribuição torna-se praticamente invariante. Na figura F.2 está ilustrado a comparação dos resultados analíticos do terceiro e quarto cumulantes entre pares ao longo de uma cadeia com a simulação numérica. 
Apêndice F. Resultados suplementares dos cumulantes da cadeia harmônica linear

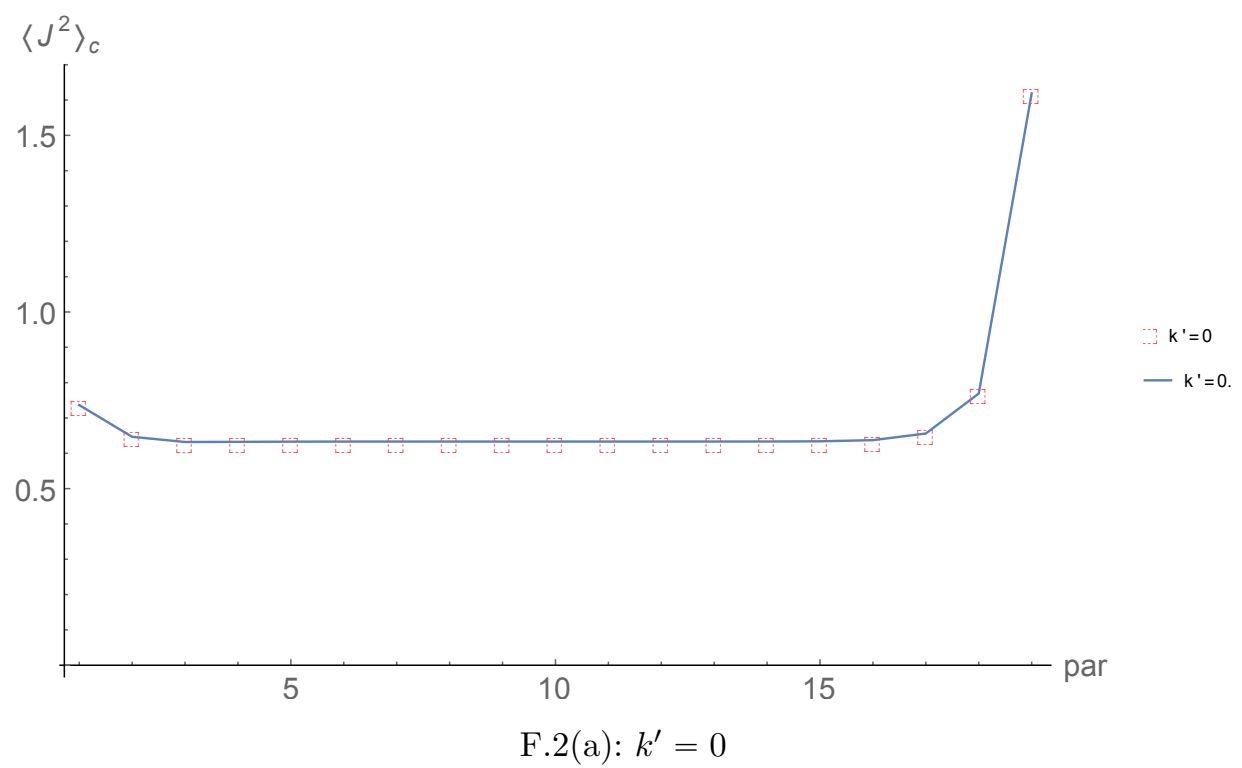

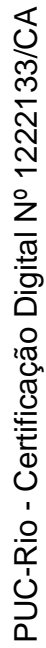

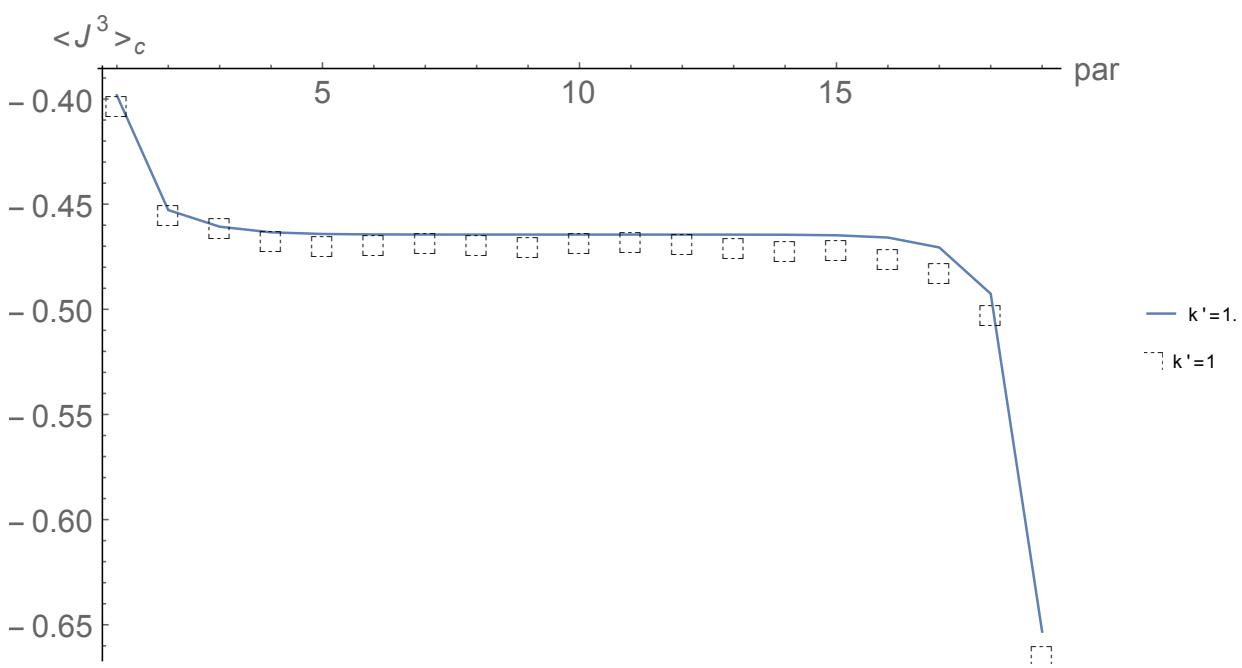

F.2(b): $k^{\prime}=1$

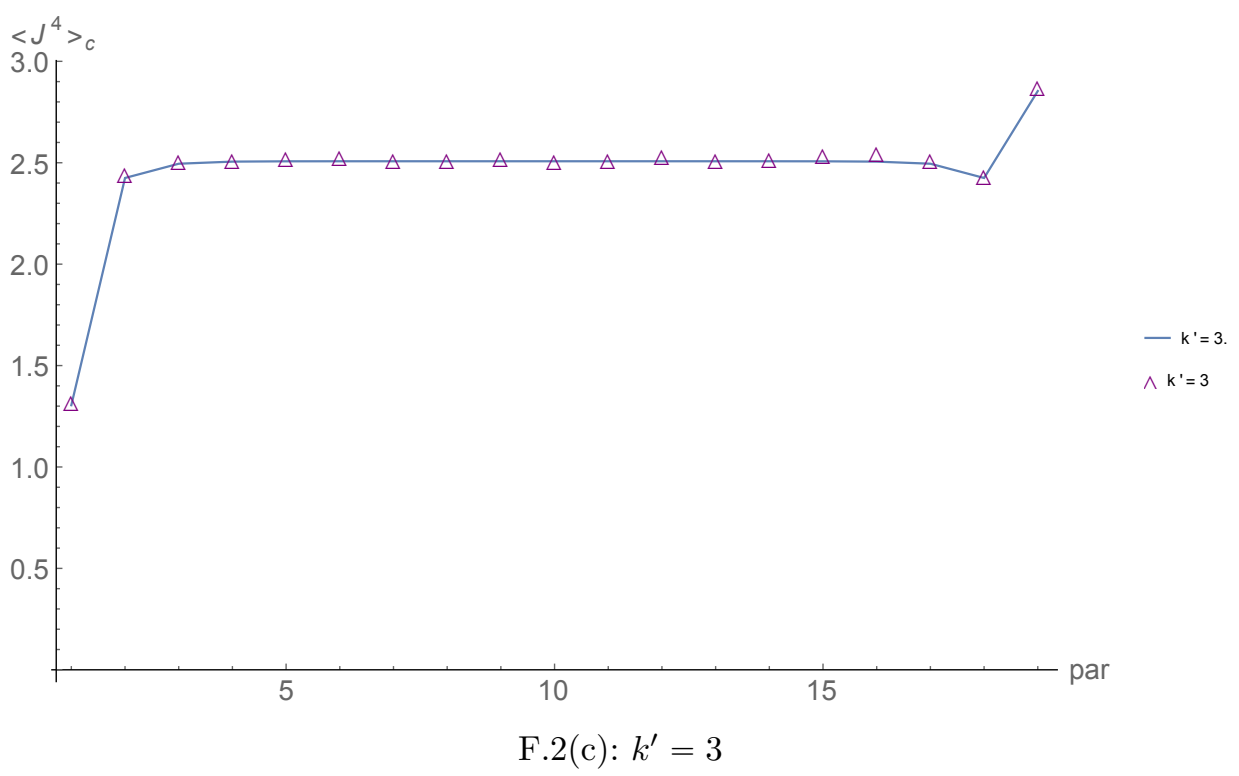

Figura F.2: As subfiguras (a), (b) e (c) mostram o comportamento do segundo, terceiro e quarto cumulantes do fluxo de calor ao longo de uma cadeia. As linhas azuis cheias mostram o resultado analítico, enquanto que os quadrados e triângulos representam o resultado da simulação. Utilizou-se $k_{1}=k^{\prime}=\gamma=$ $m=1$. 


\section{Referências bibliográficas}

[1] CALLEN, H. B. Thermodynamics and an Introduction to Thermostatistics. AAPT, 1998.

[2] HUANG, K. Introduction to statistical physics. CRC Press, 2009.

[3] REIF, F. Fundamentals of statistical and thermal physics. Waveland Press, 2009.

[4] GRIFFITHS, D. J. Introduction to electrodynamics. AAPT, 2005.

[5] STANLEY, H. Introduction to Phase Transitions and Critical Phenomena. Oxford University Press, 1971.

[6] KIPPENHAHN, R.; WEIGERT, A. ; WEISS, A. Stellar structure and evolution. Springer, 1990.

[7] THOMAS, H. Cox and Giuli's Principles of Stellar Structure. Taylor \& Francis Group, 2003.

[8] DATTA, S. Electronic transport in mesoscopic systems. Cambridge university press, 1997.

[9] KOLB, E.; TURNER, M. The Early Universe. Avalon Publishing, 1994.

[10] DEMIREL, Y. Nonequilibrium thermodynamics: transport and rate processes in physical, chemical and biological systems. Elsevier, 2007.

[11] NOVOSELOV, K. S.; GEIM, A. K.; MOROZOV, S. V.; JIANG, D.; ZHANG, Y.; DUBONOS, S. V.; GRIGORIEVA, I. V. ; FIRSOV, A. A. Science. Electric field effect in atomically thin carbon films, journal, v.306, n.5696, p. 666-669, 2004.

[12] ZHANG, Y.; TAN, Y.-W.; STORMER, H. L. ; KIM, P. Nature. Experimental observation of the quantum hall effect and berry's phase in graphene, journal, v.438, n.7065, p. 201-204, 2005.

[13] TIGHE, T.; WORLOCK, J. ; ROUKES, M. Applied physics letters. Direct thermal conductance measurements on suspended monocrystalline nanostructures, journal, v.70, n.20, p. 2687-2689, 1997. 
[14] SCHWAB, K.; HENRIKSEN, E.; WORLOCK, J. ; ROUKES, M. L. Nature. Measurement of the quantum of thermal conductance, journal, v.404, n.6781, p. 974-977, 2000.

[15] KIM, P.; SHI, L.; MAJUMDAR, A. ; MCEUEN, P. Physical review letters. Thermal transport measurements of individual multiwalled nanotubes, journal, v.87, n.21, p. 215502, 2001.

[16] DONADIO, D.; GALLI, G. Physical review letters. Thermal conductivity of isolated and interacting carbon nanotubes: comparing results from molecular dynamics and the boltzmann transport equation, journal, v.99, n.25, p. 255502, 2007.

[17] ONSAGER, L. Physical review. Reciprocal relations in irreversible processes. i., journal, v.37, n.4, p. 405, 1931.

[18] ONSAGER, L.; MACHLUP, S. Physical Review. Fluctuations and irreversible processes, journal, v.91, n.6, p. 1505, 1953.

[19] EVANS, D. J.; COHEN, E. ; MORRISS, G. Physical review letters. Probability of second law violations in shearing steady states, journal, v.71, n.15, p. 2401, 1993.

[20] CROOKS, G. E. Physical Review E. Entropy production fluctuation theorem and the nonequilibrium work relation for free energy differences, journal, v.60, n.3, p. 2721, 1999.

[21] RITORT, F. Work fluctuations, transient violations of the second law and free-energy recovery methods: Perspectives in theory and experiments. In: POINCARÉ SEMINAR 2003, p. 193-226. Springer, 2004.

[22] VAN KAMPEN, N. G.; REINHARDT, W. P. Stochastic processes in physics and chemistry. AIP, 1983.

[23] LELAND, W. E.; TAQQU, M. S.; WILLINGER, W. ; WILSON, D. V. IEEE/ACM Transactions on networking. On the self-similar nature of ethernet traffic (extended version), journal, v.2, n.1, p. 1-15, 1994.

[24] NAKAZAWA, H. Progress of Theoretical Physics Supplement. On the lattice thermal conduction, journal, v.45, p. 231-262, 1970.

[25] LEPRI, S.; LIVI, R. ; POLITI, A. Physics reports. Thermal conduction in classical low-dimensional lattices, journal, v.377, n.1, p. 1-80, 2003. 
[26] DHAR, A. Advances in Physics. Heat transport in low-dimensional systems, journal, v.57, n.5, p. 457-537, 2008.

[27] RIEDER, Z.; LEBOWITZ, J. ; LIEB, E. Journal of Mathematical Physics. Properties of a harmonic crystal in a stationary nonequilibrium state, journal, v.8, n.5, p. 1073-1078, 1967.

[28] CASATI, G.; FORD, J.; VIVALDI, F. ; VISSCHER, W. M. Physical review letters. One-dimensional classical many-body system having a normal thermal conductivity, journal, v.52, n.21, p. 1861, 1984.

[29] O'CONNOR, A. J.; LEBOWITZ, J. L. Journal of Mathematical Physics. Heat conduction and sound transmission in isotopically disordered harmonic crystals, journal, v.15, n.6, p. 692-703, 1974.

[30] PEIERLS, R. Quantum Theory of Solids. Clarendon Press, 1996.

[31] ASHCROFT, N.; MERMIN, N. Solid State Physics. Saunders College, 1976.

[32] LEPRI, S.; LIVI, R. ; POLITI, A. EPL (Europhysics Letters). On the anomalous thermal conductivity of one-dimensional lattices, journal, v.43, n.3, p. $271,1998$.

[33] LEPRI, S.; LIVI, R. ; POLITI, A. Physical review letters. Heat conduction in chains of nonlinear oscillators, journal, v.78, n.10, p. 1896, 1997.

[34] KUBO, R.; TODA, M. ; HASHITSUME, N. Statistical Physics II: Nonequilibrium Statistical Mechanics. Springer, 2012.

[35] VISSCHER, W. M.; RICH, M. Physical Review A. Stationary nonequilibrium properties of a quantum-mechanical lattice with self-consistent reservoirs, journal, v.12, n.2, p. 675, 1975.

[36] KANNAN, V. Heat conduction in low dimensional lattice systems. 2013. Tese de Doutorado - Rutgers University-Graduate SchoolNew Brunswick.

[37] TODA, M. Journal of the Physical Society of Japan. Vibration of a chain with nonlinear interaction, journal, v.22, n.2, p. 431-436, 1967.

[38] LEPRI, S. Thermal transport in low dimensions: from statistical physics to nanoscale heat transfer, 2016. 
[39] CHANG, C.-W.; OKAWA, D.; GARCIA, H.; MAJUMDAR, A. ; ZETTL, A. Physical review letters. Breakdown of fourier's law in nanotube thermal conductors, journal, v.101, n.7, p. 075903, 2008.

[40] MEIER, T.; MENGES, F.; NIRMALRAJ, P.; HÖLSCHER, H.; RIEL, H. ; GOTSMANN, B. Physical review letters. Length-dependent thermal transport along molecular chains, journal, v.113, n.6, p. 060801, 2014.

[41] LI, D.; WU, Y.; KIM, P.; SHI, L.; YANG, P.; MAJUMDAR, A. Applied Physics Letters. Thermal conductivity of individual silicon nanowires, journal, v.83, n.14, p. 2934-2936, 2003.

[42] FUJII, M.; ZHANG, X.; XIE, H.; AGO, H.; TAKAHASHI, K.; IKUTA, T.; ABE, H. ; SHIMIZU, T. Physical review letters. Measuring the thermal conductivity of a single carbon nanotube, journal, v.95, n.6, p. $065502,2005$.

[43] POP, E.; MANN, D.; WANG, Q.; GOODSON, K. ; DAI, H. Nano Letters. Thermal conductance of an individual single-wall carbon nanotube above room temperature, journal, v.6, p. 96-100, 2006.

[44] WANG, Z. L.; TANG, D. W.; LI, X. B.; ZHENG, X. H.; ZHANG, W. G.; ZHENG, L. X.; ZHU, Y. T.; JIN, A. Z.; YANG, H. F. ; GU, C. Z. Applied Physics Letters. Length-dependent thermal conductivity of an individual single-wall carbon nanotube, journal, v.91, n.12, p. 123119, 2007.

[45] DHAR, A.; DANDEKAR, R. Physica A: Statistical Mechanics and its Applications. Heat transport and current fluctuations in harmonic crystals, journal, v.418, p. 49-64, 2015.

[46] SAITO, K.; DHAR, A. Physical Review E. Generating function formula of heat transfer in harmonic networks, journal, v.83, n.4, p. 041121, 2011.

[47] HU, B.; LI, B. ; ZHAO, H. Physical Review E. Heat conduction in one-dimensional nonintegrable systems, journal, v.61, n.4, p. 3828, 2000.

[48] LEFEVERE, R.; SCHENKEL, A. Journal of Statistical Mechanics: Theory and Experiment. Normal heat conductivity in a strongly pinned chain of anharmonic oscillators, journal, v.2006, n.02, p. L02001, 2006.

[49] AOKI, K.; LUKKARINEN, J. ; SPOHN, H. Journal of statistical physics. Energy transport in weakly anharmonic chains, journal, v.124, n.5, p. 1105-1129, 2006. 
[50] DHAR, A.; ROY, D. Journal of Statistical Physics. Heat transport in harmonic lattices, journal, v.125, n.4, p. 801-820, 2006.

[51] SAITO, K. EPL (Europhysics Letters). Strong evidence of normal heat conduction in a one-dimensional quantum system, journal, v.61, n.1, p. 34, 2003.

[52] BOnETTO, F.; LEBOWITZ, J. L. ; LUKKARINEN, J. Journal of statistical physics. Fourier's law for a harmonic crystal with selfconsistent stochastic reservoirs, journal, v.116, n.1, p. 783-813, 2004.

[53] KIPNIS, C.; MARCHIORO, C. ; PRESUTTI, E. Journal of Statistical Physics. Heat flow in an exactly solvable model, journal, v.27, n.1, p. 65-74, 1982.

[54] BERNARDIN, C. Journal of Statistical Physics. Thermal conductivity for a noisy disordered harmonic chain, journal, v.133, n.3, p. 417-433, 2008.

[55] LANDI, G. T.; DE OLIVEIRA, M. J. Physical Review E. Fourier's law from a chain of coupled anharmonic oscillators under energy-conserving noise, journal, v.87, n.5, p. 052126, 2013.

[56] BOlSterli, M.; RICH, M. ; VISSCHER, W. Physical Review A. Simulation of nonharmonic interactions in a crystal by self-consistent reservoirs, journal, v.1, n.4, p. 1086, 1970.

[57] PROSEn, T.; CAMPBELL, D. K. Physical review letters. Momentum conservation implies anomalous energy transport in 1d classical lattices, journal, v.84, n.13, p. 2857, 2000.

[58] BONETTO, F.; LEBOWITZ, J. L. ; REY-BELLET, L. arXiv preprint math-ph/0002052. Fourier's law: A challenge for theorists, journal, 2000.

[59] LANDI, G. T.; DE OLIVEIRA, M. J. Physical Review E. Fourier's law from a chain of coupled planar harmonic oscillators under energy-conserving noise, journal, v.89, n.2, p. 022105, 2014.

[60] RUBIN, R. J.; GREER, W. L. Journal of Mathematical Physics. Abnormal lattice thermal conductivity of a one-dimensional, harmonic, isotopically disordered crystal, journal, v.12, n.8, p. 1686-1701, 1971.

[61] PEREIRA, E.; FALCAO, R. Physical Review E. Nonequilibrium statistical mechanics of anharmonic crystals with self-consistent stochastic reservoirs, journal, v.70, n.4, p. 046105, 2004. 
[62] CÂNDIDO, M. M.; M. MORGADO, W. A. ; DUARTE QUEIRÓS, S. M. Brazilian Journal of Physics. Eliminating the cuspidal temperature profile of a non-equilibrium chain, journal, p. "1-10", "2017".

[63] CHAudhuRI, A.; KUnDu, A.; ROY, D.; DHAR, A.; LEBOWITZ, J. L. ; SPOHN, H. Physical Review B. Heat transport and phonon localization in mass-disordered harmonic crystals, journal, v.81, n.6, p. 064301, 2010.

[64] KARDAR, M. Statistical Physics of Fields. Cambridge University Press, 2007.

[65] GOLDEnfELD, N. Lectures on Phase Transitions and the Renormalization Group. Avalon Publishing, 1992.

[66] SAITO, K.; DHAR, A. Physical review letters. Heat conduction in a three dimensional anharmonic crystal, journal, v.104, n.4, p. 040601, 2010.

[67] DHAR, A.; LEBOWITZ, J. Physical review letters. Effect of phononphonon interactions on localization, journal, v.100, n.13, p. 134301, 2008.

[68] DAS, S. G.; DHAR, A. ; NARAYAN, O. Journal of Statistical Physics. Heat conduction in the alpha- beta fermi-pasta-ulam chain, journal, v.154, n.1-2, p. 204-213, 2014.

[69] ÁvilA, R. R.; PEREIRA, E. ; TEIXEIRA, D. L. Physica A: Statistical Mechanics and its Applications. Length dependence of heat conduction in (an)harmonic chains with asymmetries or long range interparticle interactions, journal, v.423, p. $51-60,2015$.

[70] MEndonÇA, M. S.; PEREIRA, E. Physics Letters A. Effective approach for anharmonic chains of oscillators: Analytical description of negative differential thermal resistance, journal, v.379, n.36, p. 1983-1989, 2015.

[71] FALCAO, R.; NETO, A. F. ; PEREIRA, E. Theoretical and Mathematical Physics. Analytic approach to the (an) harmonic crystal chains with self-consistent stochastic reservoirs, journal, v.156, n.1, p. 1081-1088, 2008.

[72] GENDELMAN, O.; SAVIN, A. Physical review letters. Normal heat conductivity of the one-dimensional lattice with periodic potential of nearestneighbor interaction, journal, v.84, n.11, p. 2381, 2000. 
[73] OlivareS, C.; AnteneOdO, C. Physical Review E. Role of the range of the interactions in thermal conduction, journal, v.94, n.4, p. 042117 , 2016.

[74] DHAR, A. Physical review letters. Heat conduction in the disordered harmonic chain revisited, journal, v.86, n.26, p. 5882, 2001.

[75] BARros, F.; Lemos, H. C. ; PEREIRA, E. Physical Review E. Changing the heat conductivity: An analytical study, journal, v.74, n.5, p. 052102, 2006.

[76] BOnETTO, F.; LEBOWITZ, J. L. ; LUKKARINEN, J. Journal of statistical physics. Fourier's law for a harmonic crystal with selfconsistent stochastic reservoirs, journal, v.116, n.1, p. 783-813, 2004.

[77] FERMI, E.; PASTA, J. ; ULAM, S. Studies of nonlinear problems I, Los Alamos Report LA 1940, 1955. In: REPRODUCED IN NONLINEAR WAVE MOTION. Amer. Math. Soc., 1974.

[78] MORGadO, W. A.; QUEIRÓS, S. M. D. Physical Review E. Role of the nature of noise in the thermal conductance of mechanical systems, journal, v.86, n.4, p. 041108, 2012.

[79] MOrgado, W. A.; Queirós, S. M. D. Physical Review E. Thermostatistics of small nonlinear systems: Gaussian thermal bath, journal, v.90, n.2, p. 022110, 2014.

[80] MORGADO, W. A.; QUEIRÓS, S. D. ; SOARES-PINTO, D. O. Journal of Statistical Mechanics: Theory and Experiment. On exact time averages of a massive poisson particle, journal, v.2011, n.06, p. P06010, 2011.

[81] DRUZHInINA, T. S.; HOEPPENER, S. ; SCHUBERT, U. S. Nano letters. Microwave-assisted fabrication of carbon nanotube afm tips, journal, v.10, n.10, p. 4009-4012, 2010.

[82] KOGAN, S. Electronic noise and fluctuations in solids. Cambridge University Press, 2008.

[83] REIMANN, P. Physics reports. Brownian motors: noisy transport far from equilibrium, journal, v.361, n.2, p. 57-265, 2002.

[84] CZERnIK, T.; KULA, J.; ŁUCZKA, J. ; HÄNGGI, P. Physical Review E. Thermal ratchets driven by poissonian white shot noise, journal, v.55, n.4, p. 4057, 1997. 
[85] BAUlE, A.; COHEN, E. Physical Review E. Fluctuation properties of an effective nonlinear system subject to poisson noise, journal, v.79, n.3, p. 030103, 2009.

[86] ANDERSEN, H. C. The Journal of chemical physics. Molecular dynamics simulations at constant pressure and/or temperature, journal, v.72, n.4, p. 2384-2393, 1980.

[87] KANAZAWA, K.; SAGAWA, T. ; HAYAKAWA, H. Physical review letters. Stochastic energetics for non-gaussian processes, journal, v.108, n.21, p. 210601, 2012.

[88] KANAZAWA, K.; SAGAWA, T. ; HAYAKAWA, H. Physical Review E. Heat conduction induced by non-gaussian athermal fluctuations, journal, v.87, n.5, p. 052124, 2013.

[89] ESHUIS, P.; VAN DER WEELE, K.; LOHSE, D. ; VAN DER MEER, D. Physical review letters. Experimental realization of a rotational ratchet in a granular gas, journal, v.104, n.24, p. 248001, 2010.

[90] PEKOLA, J. P. Physical review letters. Josephson junction as a detector of poissonian charge injection, journal, v.93, n.20, p. 206601, 2004.

[91] Filatrella, G.; PIERRO, V. Physical Review E. Detection of noise-corrupted sinusoidal signals with josephson junctions, journal, v.82, n.4, p. 046712, 2010.

[92] BEN-ISAAC, E.; PARK, Y.; POPESCU, G.; BROWN, F. L.; GOV, N. S. ; SHOKEF, Y. Physical review letters. Effective temperature of red-blood-cell membrane fluctuations, journal, v.106, n.23, p. 238103, 2011.

[93] MORGado, W. A.; Queirós, S. M. D. Physical Review E. Thermostatistics of small nonlinear systems: Poissonian athermal bath, journal, v.93, n.1, p. 012121, 2016.

[94] MimnaGH, D.; BALlentine, L. Physical Review E. Thermal conductivity in a chain of alternately free and bound particles, journal, v.56, n.5, p. 5332, 1997.

[95] MARCINKIEWICZ, J. Mathematische Zeitschrift. Sur une propriété de la loi de gauß, journal, v.44, n.1, p. 612-618, 1939.

[96] TERraneO, M.; PEYRARD, M. ; CASATI, G. Physical Review Letters. Controlling the energy flow in nonlinear lattices: a model for a thermal rectifier, journal, v.88, n.9, p. 094302, 2002. 
[97] WANG, L.; LI, B. Phys. Rev. Lett. Thermal logic gates: Computation with phonons, journal, v.99, p. 177208, 2007.

[98] CASATI, G. Nature nanotechnology. Device physics: The heat is on-and off, journal, v.2, n.1, p. 23, 2007.

[99] LIPPI, A.; LIVI, R. Journal of Statistical Physics. Heat conduction in two-dimensional nonlinear lattices, journal, v.100, n.5-6, p. 1147-1172, 2000.

[100] YAnG, L.; GRASSBERGER, P. ; HU, B. Physical Review E. Dimensional crossover of heat conduction in low dimensions, journal, v.74, n.6, p. 062101, 2006.

[101] SHIBA, H.; ITO, N. Journal of the Physical Society of Japan. Anomalous heat conduction in three-dimensional nonlinear lattices, journal, v.77, n.5, p. 054006-054006, 2008.

[102] VAN DER POL, B.; BREMMER, H. Operational Calculus: Based on the Two-sided Laplace Integral. University Press, 1955.

[103] USMANI, R. Computers \& Mathematics with Applications. Inversion of jacobi's tridiagonal matrix, journal, v.27, n.8, p. 59 - 66, 1994.

[104] ELLIOTT, J. F. The characteristic roots of certain real symmetric matrices. 1953. Dissertação de Mestrado - University of Tennessee. 